\title{
Connectivity and auditory verbal hallucinations : contributions of functional imaging and psychometric studies
}

Citation for published version (APA):

van de Ven, V. G. (2006). Connectivity and auditory verbal hallucinations : contributions of functional imaging and psychometric studies. [Doctoral Thesis, Maastricht University]. Datawyse / Universitaire Pers Maastricht. https://doi.org/10.26481/dis.20060127vv

Document status and date:

Published: 01/01/2006

DOI:

10.26481/dis.20060127vv

Document Version:

Publisher's PDF, also known as Version of record

\section{Please check the document version of this publication:}

- A submitted manuscript is the version of the article upon submission and before peer-review. There can be important differences between the submitted version and the official published version of record.

People interested in the research are advised to contact the author for the final version of the publication, or visit the DOI to the publisher's website.

- The final author version and the galley proof are versions of the publication after peer review.

- The final published version features the final layout of the paper including the volume, issue and page numbers.

Link to publication

\footnotetext{
General rights rights.

- You may freely distribute the URL identifying the publication in the public portal. please follow below link for the End User Agreement:

www.umlib.nl/taverne-license

Take down policy

If you believe that this document breaches copyright please contact us at:

repository@maastrichtuniversity.nl

providing details and we will investigate your claim.
}

Copyright and moral rights for the publications made accessible in the public portal are retained by the authors and/or other copyright owners and it is a condition of accessing publications that users recognise and abide by the legal requirements associated with these

- Users may download and print one copy of any publication from the public portal for the purpose of private study or research.

- You may not further distribute the material or use it for any profit-making activity or commercial gain

If the publication is distributed under the terms of Article $25 \mathrm{fa}$ of the Dutch Copyright Act, indicated by the "Taverne" license above, 


\section{Connectivity and auditory verbal hallucinations}

Contributions of functional imaging and psychometric studies 
की

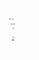

Y

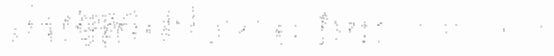




\section{Connectivity and auditory verbal hallucinations}

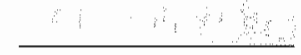 \\ Contributions of functional imaging and psychometric studies}

Proefschrift

Ter verkrijging van de graad van doctor aan de Universiteit Maastricht, op gezag van de Rector Magnificus, Prof. Mr. G.P.M.F. Mols

volgens het besluit van het College van Decanen, in het openbaar te verdedigen op vrijdag 27 januari 2006 om 14:00 uur

door

Vincent Gordon van de Ven 


\section{Promotor}

Prof dr. R. Goebel

\section{Copromotores}

Dr. E. Formisano

Dr. D.E.J. Linden (University of Wales, U.K.)

\section{Beoordelingscommissie}

Prof. dr. J. Jolles (voorzitter)

Dr. A. Aleman (Rijksuniversiteit Groningen)

Prof. dr. J. van Os

Prof. dr. N.O. Schiller

Prof. dr. F. Schneider (Universitätsklinikum Aachen, Duitsland)

Research reported in chapters $1,2,3$ and 5 was conducted at the Laboratory for Neurophysiology, Department of Psychiatry, Johann Wolfgang Goethe University, Frankfurt am Main, Germany. Research in chapter 4 was conducted at the Department of Neuroradiology, Universitá Federico II, Naples, Italy and in Frankfurt. The analysis reported in chapter 6 was conducted at the University of Maastricht, Maastricht, The Netherlands.

ISBN 90-9020324-9

Copyright $($ Vincent van de Ven. All rights reserved.

Cover design: Vincent van de Ven \& Ingrid Christoffel. 
Opgedragen aan mijn opa, Wim Weber ... unforgettable in every way. 
$\therefore$

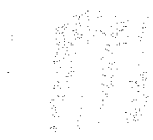

क क

$\because$ 


\section{Table of contents}

Introduction

Chapter 1 Functional connectivity as revealed by spatial independent component analysis of fMRI measurements during rest

Chapter 2 The spatiotemporal pattern of auditory cortical responses during verbal hallucinations

Chapter 3 Exploring connectivity of auditory verbal hallucinations 61

Chapter 4 Components of target and distractor processing using selforganizing group-level ICA

Chapter 5 Enhanced vividness of mental imagery as a trait marker of schizophrenia?

Chapter 6 Crystallized intelligence, perceptual abilities and symptoms of schizophrenia: An exploratory approach

Summary

121

Samenvatting

Color figures

Abbreviations

References

Acknowledgements

Publications 
$\therefore \quad \therefore \quad 3$

$\therefore \quad$

$\therefore=$ 


\section{Introduction}

Auditory verbal hallucinations (AVH) are the perception of voices in the absence of corresponding agents in the external world. Hallucinations are a cardinal symptom in the diagnosis of schizophrenia, with a lifetime prevalence of over $65 \%$. In many cases the voices cause considerable distress and anxiety because they provide derogatory commentary, command the patient to engage in shameful, dangerous or otherwise undesirable behaviours, or talk about the patient with other voices. AVH also occur in the context of other psychiatric or neurological disorders, and may be the result of drug abuse. However, AVH do not seem to be confined to the pathological domain, but are also reported by non-clinical individuals. Indeed, non-clinical hallucinations have been the subject of scientific study at least since the time of Galton (1883). It has been suggested that hallucinations exist on a continuum from relatively benign and non-clinical (occurring in the healthy population and scoring high on schizotypal traits) to pathological manifestations as witnessed in schizophrenia. Nonetheless, it is not clear if non-clinical hallucinations share etiological grounds with clinical hallucinations.

Many cognitive and neuroanatomical studies have focused on the perceptual aspects of AVH. For example, hallucinations are hypothesized to be related to the vividness of mental imagery. Mental imagery is defined as the ability to mentally "visualize" an object or scene in any sensory modality, as well as in the motor domain'. Examples are seeing a friend's face before the mind's eye when looking for this friend in a crowd of people, or hearing a popular song ring through your head. Mental imagery contributes to many cognitive abilities, such as learning, memory and attention. Mental imagery holds in common with hallucinations the perceptual nature, but is clearly distinguished by the degree of volition that is excercised, as well as the experienced vividness of the percepts. Healthy subjects are able to mentally visualize according to their own decision, and their visualized images are typically rated as less vivid than real, sensory-driven perceptual experiences. However, several theoretical and empirical studies have shown that hallucinations may bear on an increased vividness of mental images (and lack of control of these images), which in combination with impairments in other

\footnotetext{
' Here, it is assumed that mental imagery shares cognitive and neural processes with normal perception. It should be noted that this is a widely, but not unanimously held position within the literature.
} 
cognitive systems, may blur the distinction between real and imagined percepts. However, an equally large amount of studies provided evidence to the contrary, that is, a lack of or reversed association between hallucinations and imagery vividness.

In addition, AVH have been associated with impairments in non-sensory, higher order systems, such as language processing, monitoring of internally generated events or memory. For example, hallucinations may be the result of an impairment in self-monitoring, which is the cognitive ability to identify the source of events or actions as self-generated or elicited by an external agent. Hallucinating patients tend to attribute an external source to items or events they have generated themselves. Neuro-anatomical and neurophysiological candidates for a mechanism that controls monitoring of self-generated events have been proposed to reside in connections between frontal and temporal cortical areas. In AVH, the impairment of this mechanism may be manifested through a failure to provide a neural copy of the self-generated event (termed efference copy). At the same time, AVH may be associated with impairments in language processing, perhaps caused by a disconnection (i.e., impaired functional and anatomical connections) between language production and perception areas, where it is proposed that in healthy subjects the perception areas are inhibited when the brain generates its own speech. It is conceivable that a combination of impairments in these cognitive systems may underlie AVH in schizophrenia.

On the neurobiological level a small number of structural imaging studies revealed decreased amount of gray matter in schizophrenia in the left primary auditory cortex and superior temporal gyrus, which is important for complex analyses of auditory signals (e.g., language, object identity). These structural changes are correlated with the presence and severity of auditory hallucinations. At the same time, it has been proposed that hallucinations, as well as other psychotic symptoms, are associated with a decreased asymmetry of the planum temporale, or temporal plane, in schizophrenia. In healthy subjects the planum temporale, which is seated on top of the superior temporal gyrus and inside the sylvian fissure, is larger in the left hemisphere in comparison to the right (hemispheric asymmetry), and subserves language perception and processing. The decrease, or even reversal of planum temporale asymmetry may be associated with impairments in language processing and monitoring. However, the exact relation between structure and hallucinations remains to be elucidated.

\section{Functional magnetic resonance imaging}

The study of hallucinations has received new and innovative impulses from the development of functional imaging techniques, where it is possible to measure brain activity in-vivo. Of most importance to this thesis is functional magnetic resonance imaging (AMRI), which measures brain activity non-invasively. The 
whole brain or parts thereof are measured in an extremely large set of threedimensional (3-D) spatial units (voxels) of a few millimeter in each dimension, constituting a very high spatial resolution or detailedness. A whole-brain measurement can be achieved within one or several seconds, constituting a moderate temporal resolution in comparison to the superior resolution of milliseconds in electroencephalography. Because the measurement can be repeated a large number of times brain activity can be sampled to a high degree, increasing the statistical power of the method. The consecutive measurements for each voxel, constitute the voxel timecourse, representing the changes in brain activity over time for a spatial location.

The vast majority of fMRI studies uses localized blood flow as an indirect measure of brain activity, relying on the coupling between changes in neuronal activity and blood flow. With increased neuronal firing more oxygen-enriched blood flows into the area of increased neuronal activity. The resulting change in magnetic properties of the blood flow in this brain area forms the basis of the measured signal, termed the blood-oxygen-level dependent (BOLD) contrast. Thus, in fMRI increased neuronal activity is represented by an increase in signal amplitude. Typically, the BOLD signal is sluggish and delayed by several seconds with respect to the neuronal activity.

With the advent of fMRI in the study of hallucinations new insights have been obtained, leading to a better understanding about the neural underpinnings of the aberrant perceptual experience. However, much controversy remains to be solved. One of the challenges has been the issue of the involvement of primary sensory areas in hallucinations. Many studies found evidence that activity in primary visual and auditory cortex could be significantly increased during sensory-specific attention, but only primary visual cortex has been found activated during mental imagery. In 1999, Dierks and colleagues published a report of increased activity in primary auditory cortex during AVH by using fMRI, and made the intriguing suggestion that this activity may reflect the increased vividness that is commonly associated with such hallucinations. Primary auditory cortex does not seem to be active during auditory mental imagery in healthy individuals, where the internally invoked auditory images are experienced as less vivid than sensory-driven, externally evoked percepts.

\section{Issues addressed in this thesis}

Activity in primary auditory cortex during AVH has been found by only a few studies, while a number of other studies did not find this activity. The inconsistency of these findings may be partially confounded by modelling the hypothesized brain activity related to hallucinations according to the patient self-reports on time of onset and duration of their hallucinations. These selfreports may not be accurate enough to capture all interesting activity patterns. Furthermore, the activity in primary auditory cortex may show an unexpected 
pattern in neuronal or hemodynamic terms. Brain activity (neuronal) or other parameters that influence the BOLD response (blood flow parameters, capillary behaviour) may be altered in schizophrenia, with respect to heal thy individuals, because of disease- or medication-specific reasons. Such differences may affect the measured BOLD signal related to hallucinations, for example, an early transient response that does not coincide with the length of the experienced episodes of hallucinations. Therefore, to understand how hallucinations arise in the brain, it is imperative to acquire knowledge about the differences in "default" functional brain states in healthy and pathological brains.

Secondly, all studies of hallucinations have so far used activity-related analyses, where brain areas are localized that show a hypothesized model of activity. The model is typically applied to the functional data in the timedomain, in a voxel-by-voxel manner, where each test is treated independently from all other tests. Voxel timecourses that are significantly associated with the model are identified and coloured, resulting in a spatial (colour) map of significantly activated voxels. In contrast, brain activity related to hallucinations has not been analysed on a connectivity-related level, where the spatiotemporal relations between (spatially separate) brain areas is investigated, where brain areas can be defined as single voxels, clusters of voxels or networks of such clusters. Connectivity-related analyses reveal information on how brain areas communicate. The simplest form of connectivity is a correlation between the activity patterns of two brain areas, which is termed functional connectivity. In essence, functional connectivity is an estimate of to what degree spatially segregated brain areas are active in synchrony. It must be noted that by definition it does not reveal any information about the direction (i.e., causality) or nature of the connection between a pair of brain areas.

How can the concept of functional connectivity tell us anything about the brain's activity during the experience of hallucinations? Functional connectivity as defined above does not require the use of an a priori defined model of brain activity (in constrast to hypothesis-driven analysis), which has important consequences for analysis and interpretation. Because the analysis does not depend on assumptions of brain activity it is possible to circumvent the potential drawbacks that are related to patient self-reports and the use of a single set of parameters to describe the BOLD response for all brain areas. Moreover, such an analysis may provide insight into previously unmodelled activity related to hallucinations. While some areas show a temporal profille similar to the sequences of hallucinatory experiences in terms of their timing and duration, it may be possible that other areas only show transient activity at the onsets of a hallucination, or show initial activity before the hallucination reaches conscious awareness. Thus, by using an approach that considers the relations between brain areas independently of a predefined model it may be possible to disentangle brain areas, or networks of brain areas, with unique temporal signatures that are associated with the hearing of "voices". 


\section{Overview}

This thesis explores the use of multivariate methods in the studly of auditory verbal hallucinations. The main motivation was to investigate neural, as well as neuropsychological "networks" that underlie the experience and occurrence of AVH in schizophrenia. The thesis presents two main approaches: if) connectivity analysis of neural activity, and 2) multivariate analyses of neuropsychological, cognitive and symptomatic aspects related to hallucinations. The main multivariate approach for the analysis of functional imaging data that is used in this thesis is independent component analysis (ICA), which was introduced into the field of functional neuroimaging several years ago. However, neuropsychological assessments were also subdued to multivariate analyses, in an attempt to characterize the relations between hallucinations, mental imagery and a small set of cognitive and perceptual abilities.

Chapter one introduces and describes spatial ICA as a tool for analysing patterns of functional connectivity in a situation where no a priori model about regional brain activity can be generated. There are different analytical tools available for the assessment of functional connectivity. However, the tools of choice in this thesis can be used in cases when no ideal model of brain activity is present, or when such a model may not be sensitive enough to capture all relevant patterns of brain activity. This study focuses on patterns of connectivity that are present when subjects do not perform any specified task. Such a state is commonly used as a resting or baseline condition in functional imaging studies, where brain activity during experimental conditions is contrasted to the activity during rest. However, it has been known for many decades that the resting brain does not show an absence of activity. On the contrary, the resting brain shows regionally specific patterns of activity that may represent the maintenance of a "default state". This approach may ultimately be used to assess differences in default state across different populations.

Chapter two is a first application of spatial ICA to functional imaging data of AVH. This study focused on the specific question if primary auditory cortex is activated during the experience of auditory hallucinations. The study was partly motivated by the errors or lack of sensitivity that may potentially be present when modeling brain activity solely via patients' self-reports on the timing of their hallucinations. The spatiotemporal activity pattern of the auditory cortex during hallucinations was analysed using a combination of independent component analysis and visualization tools that have been developed recently in the field of functional neuroimaging.

Chapter three is an extension of the ICA application discussed in the previous chapter. In chapter three the functional data of five hallucinating schizophrenia patients are probed for multiple networks that may underlie the experience of AVH. In addition to auditory cortex, networks of fronto-temporal 
areas that are known to be involved in language processing and-monitoring are identified and further analysed using post-hoc methods, which include estimation of timing differences in signal increases, and spatial lateralization (i.e, the degree to which these functional networks are localized to a specific hemisphere). The presence of these networks in separate components may be an indication that sensory- and language-related systems may be active in a cooperative fashion, but contribute differently to the experience of hallucinations.

Ultimately, a group result of functional connectivity states, represented by the spatial ICA maps, is desired, in order to compare connectivity states between schizophrenia and a control group. However, this issue has proved to be far from trivial, where one of the core problems is whether to retain subjectspecific spatiotemporal information, or to base the connectivity analysis on aggregate data.

In chapter four the issue of group analysis using spatial ICA is investigated. In this chapter, a combination of single-subject spatial ICA and a clustering algorithm within the subject space, which preserves subject-specific information, is applied to data of target detection in healthy individuals. Different attentional networks that may be involved in the detection of infrequent target stimuli within a set of frequent and infrequent irrelevant stimuli are investigated, and their spatial consistency across subjects is assessed. These results can be compared to connectivity patterns in hallucinating patients in future studies.

Chapter five presents the investigation of the association between the presence and severity of auditory and visual hallucinations and vividness of mental imagery. Predisposition to hallucinate and vividness of mental imagery were obtained by self-report questionnaires from paranoid schizophrenia patients and healthy controls. At the same time, cognitive and perceptual ability tasks were performed and individual psychopathology ratings were obtained from the patients, and were treated as covariates.

Several studies have indicated that impairments in performance of a wide variety of neuropsychological tests observed with schizophrenia patients do not represent a wide array of independent cognitive impairments, but rather show a specific pattern that suggests the presence of a common pathological denominator. The small test battery that was used in the study described in chapter five comprised tests for crystallized intelligence, fluid intelligence in terms of perceptual abilities, and psychomotor speed. Crystallized and fluid intelligence are assumed to be uncorrelated components of intelligence in healthy individuals.

In chapter six the data were reanalyzed using explorative principal component analysis (PCA) in order to verify whether the schizophrenia group showed the same dichotomy in cognitive ability scores, as commonly observed in healthy control groups, and whether the PCA results showed any relation to symptom ratings. 
The last chapter provides a summary of the results and conclusions of the previous chapters, and suggests new lines of research for the further investigation of auditory hallucinations in schizophrenia patients, as well as in other populations. 


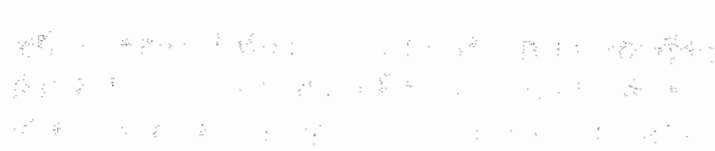




\section{Chapter 1}

\section{Functional connectivity as revealed by spatial independent component analysis of fMRI measurements during rest}

Based on publication:

Van de Ven, V.G., Formisano, E., Prvulovic, D., Röder, C.H. \& Linden, D.E.J. (2004). Functional connectivity as revealed by spatial independent component analysis of fMRI measurements during rest. Human Brain Mapping, 22, 165-178. 


\section{Abstract}

Cortical functional connectivity, as indicated by the concurrent spontaneous activity of spatially segregated regions, is increasingly being studied because a) it may determine the reaction of the brain to external stimuli and task requirements and b) it is supposed to be altered in many neurological and psychiatric disorders. In functional MRI (fMRI), such functional connectivity is commonly investigated by correlating the timecourse of a chosen "seed voxel" with the remaining voxel timecourses in a voxel by voxel manner. Yet, this approach is biased by the actual choice of the seed voxel, because it only shows functional connectivity for the chosen brain region, while ignoring other potentially interesting patterns of coactivation. In this study we used spatial independent component analysis (sICA) to assess cortical functional connectivity maps from resting state data. SICA does not depend on any chosen temporal profile of local brain activity. We hypothesized that sICA would be able to find functionally connected brain regions within the sensory and motor regions in the absence of task-related brain activity. We also investigated functional connectivity patterns of several parietal regions including the superior parietal cortex and the posterior cingulate gyrus. The components of interest were selected in an automated fashion using predefined anatomical volumes of interest. SICA yielded connectivity maps of bilateral auditory, motor and visual cortices. Moreover, it showed that prefrontal and parietal areas, too, are functionally connected within and between hemispheres during the resting state. These connectivity maps showed an extremely high degree of consistency in spatial, as well as in temporal and frequency parameters within and between subjects. These results are discussed in the context of the recent debate on the functional relevance of fluctuations of neural activity in the resting state. 


\section{Introduction}

Functional neuroimaging is increasingly applied to issues of connectivity and communication between distant areas of the brain. While functional connectivity refers to the correspondence over time between spatially distinct neurophysiological events without implying any notion of directionality, effective connectivity refers to the influence one brain region exerts over another (Friston, 1996). Functional connectivity can be assessed by cognitive or motor tasks (Friston, 1996; Goebel et al., 1998), but it can also be extracted from the spontaneous activity of the resting brain. In the latter case a network of functional communication between areas is revealed when their ongoing activity is not altered through task or cognitive demand. Compared to taskrelated functional connectivity, resting state connectivity patterns probably depend more on a common neural modulator (e.g. thalamic input into cortical regions) or inherent neurophysiological properties of cortico-cortical interaction (e.g. synchronized spontaneous neural firing). With electroencephalography (EEG) (Tucker et al., 1986) and direct neuronal recording (Fries et al., 2001; Leopold et al., 2003) it has been documented that, during resting states, cortical neurons belonging to specific but spatially separated functional clusters show correlated patterns of spontaneous activity over time. Distinctive spatial distributions of correlated inter-hemispheric activity have been found for motor, auditory and visual cortices (e.g. Shen et al., 1999; Tucker et al., 1986). Such functional connectivity maps may provide insights into the anatomicalfunctional relations that are not modulated by cognitive or motor task performance (Greicius et al., 2003).

The functional connectivity of the resting brain has also been studied with functional magnetic resonance imaging (fMRI). The blood oxygen leveldependent (BOLD) signal of $\mathrm{FMRI}$ has been confirmed to reflect neural activity (Logothetis et al., 2001), which is a prerequisite for the assumption that BOLD signal functional connectivity maps may represent correlated neural activity of spatially segregated brain regions (Leopold et al., 2003). However, caution is needed when interpreting functional connectivity in fMRI since many other metabolic processes and local hemodynamic properties at various time scalles may contribute to the structure of BOLD signal fluctuations (Mitra et al.., 1997).

The traditional method to assess functional connectivity maps for a specific region of interest in FMRI is the seed-voxel approach. One or a small cluster of voxels is chosen, whose (averaged) timecourse serves as a reference model for cross-correlation analysis with the remaining brain voxels, yielding a spatial zero-lag cross-correlation map (Biswal et al, 1995; Goebel et al., 1998). The seedvoxel can be selected using anatomical (e.g., gyral or sulcal landmarks) or functional knowledge (e.g., centre of mass of clusters of statistical maps). Using this approach, functional connectivity maps depicting bilateral auditory (Biswal et al., 1996 [abstract]), visual (Lowe et al., 1998) and motor cortex (Cordes et al., 
2001; Lowe et al., 1998; Xiong et al., 1999) that match statistical maps of sensory or motor tasks have been obtained and replicated. In addition, it has been reported that low-frequency oscillations in the range of $0.1 \mathrm{~Hz}$ or lower contributed most to the temporal structure of the auditory, visual and sensorimotor functional connectivity maps (Cordes et al., 2001).

However, the seed-voxel approach has some inherent caveats. Firstly, the cross-correlation map that is obtained depends highly on the strategies for choosing the seed-voxel, which determine the reference model. In addition, for resting state analyses the chosen model will contain a high but unknown degree of noise-related fluctuations. Secondly, as an instance of the univariate general linear model (GLM: Friston, 1996) the cross-correlations are computed on a voxel by voxel basis, thus pairing one independent (seed-voxel timecourse) and one dependent (timecourse of a remaining voxel) variable. This approach thus ignores the relations between multiple voxels.

Several multivariate, model-independent methods have been applied to resting state data in order to circumvent the problems of the seed-voxel approach. These methods do not depend on the choice of any seed-voxel reference, but rather use the covariance matrix of all implicated voxel timecourses as the starting point for analysis. However, many of these methods use a subset of cortical voxels to estimate functional connectivity networks. In this sense, hierarchical clustering has been used in resting state measurements to assess networks of functional connectivity (Cordes et al., 2002). Clustering techniques generate clusters of variables based upon an index of similarity or difference that can represent absolute (e.g., Euclidean distance) or relative units (e.g., Pearson correlation). Unlike other clustering techniques, hierarchical clustering does not depend on a predefined number of clusters to be estimated. In the study by Cordes et al. (2002), clusters of interest were chosen according to low-frequency contributions to the timecourse of the cluster centre, which yielded connectivity maps similar to those found with the seed-voxel approach. Another approach to assess functional connectivity is principal component analysis (PCA: Friston et al., 1993). PCA estimates a number of orthogonal components from the covariance structure that together explain a large part of the variance of the data. Although these methods could replicate the functional connectivity maps obtained with the seed-voxel approach, they have some limitations. First, often a subset of brain voxels is chosen from which the covariance matrix is estimated, thus potentially biasing the analysis. Second, PCA considers only second-order (and not higher-order) relations between voxels and the time-courses of the components are constrained to be orthogonal. A useful candidate method to circumvent these drawbacks is independent component analysis (ICA), a data-driven, multivariate signal processing approach (Comon, 1994). In the applications of ICA to fMRI data, the observed 4D signals are usually modeled as linear "mixtures" of unknown spatially independent processes (e.g. BOLD fluctuations, head movements, artifacts, etc), each contributing to the data set with an unknown time profile (spatial ICA or sICA: McKeown et al., 1998a,b; McKeown \& Sejnowski, 1998). 


\section{Chapter 1}

The time-series are decomposed into spatial components or modes (ICs) that each have a unique time-course (TC). The decomposition process, for which different algorithms have been developed (Bell \& Sejnowski, 1995; Hyvärinen, 1999), maximizes the spatial statistical independence of the components. It is important to note that the spatial independence of the components is a much stronger criterion than if the spatial maps, or eigenimages, were merely constrained to be orthogonal (as in PCA). In this sense, sICA can be considered

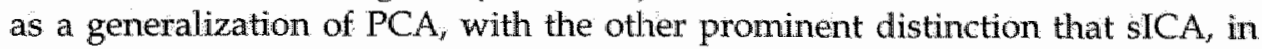
contrast to PCA, does not constrain the time-courses of the components to be mutually orthogonal.

Spatial ICA has been applied to resting state data of anesthetized child patients by Kiviniemi et al. (2003). These authors were able to identify components in sensory and motor cortices and large vessels. The frequency analysis of parenchymal time courses showed a dominant peak at $0.03 \mathrm{~Hz}$. ICA has also been applied to resting state data of the adult human brain (Biswal \& Ulmer, 1999), but in this study no thorough analysis of functional connectivity patterns was reported. We apply this method to fMRI data of healthy adults in order to study resting state functional connectivity of the non-anesthetized human brain. We show that spatial ICA can be used to assess functional connectivity within and across hemispheres in sensory and motor cortices, as well as in a number of parietal and frontal regions. In addition, we assess the degree of reproducibility of these functional connectivity maps within and between subjects, by analyzing their spatial layout and their temporal profile.

\section{Methods}

\section{Subjects}

Seven healthy subjects with no history of neurological or psychiatric disorder participated in the study ( 4 males; mean age $=28.6$ years, range $=22-39$ ). Two subjects were predominantly left-handed, the remaining were predominantly right-handed, as assessed using the Edinburgh Handedness Inventory (Raczkowski et al., 1974). The aim of the study was explained to the subjects and all subjects gave written informed consent prior to measurement. Before functional and anatomical measurements the subjects were told to relax but remain awake, keep their eyes open and just to "let your thoughts go as they come". Subjects were asked between and after sessions whether they were awake during the previous session. All subjects confirmed they were.

\section{Imaging sequences and parameters}

Functional and anatomical measurements were conducted on a 1.5 T Magnetom Vision MRI Tomograph (Siemens, Erlangen, Germany) using an EPI sequence for functional imaging ( 1 volume $=12$ axial slices, number of volumes $=400$, $\mathrm{TR} / \mathrm{TE}=1500 / 60 \mathrm{~ms}, \mathrm{FoV}=220 \times 220 \mathrm{~mm}^{2}$, voxel size $=3.2 \times 3.2 \times 6.0 \mathrm{~mm}^{3}$ ). 


\section{Functional connectivity during rest}

Each functional session lasted $10 \mathrm{~min}$. For each subject a T1-weighted MPRAGE (magnetization-prepared rapid gradient echo) sequence was run to obtain an anatornical reference (voxel size $=1.0 \times 1.0 \times 1.0 \mathrm{~mm}^{3}, \mathrm{FoV}=256 \times 256$ $\mathrm{mm}^{2}$ ), which lasted appr. $5 \mathrm{~min}$. In order to prevent large head movements the subject's head was fixed by placing foam pads on each side of the head within the head-cage. For each subject, the session began with one functional measurement of resting state, followed by an anatomical measurement, which was followed by a second functional measurement. Functional imaging was performed in complete darkness, and subjects were told they should keep their eyes open in order to prevent them from falling asleep during the measurements. Subjects were also explicitly instructed to move as little as possible.

\section{Functional connectivity analysis}

Analysis of the measured BOLD signals was carried out using the BrainVoyager 2000 v.4.8 analysis software (www.brainvoyager.com). The first 4 volumes of each functional data set were discarded from analysis because of magnetic saturation effects. All functional datasets were pre-processed using inter-slice time correction and resampled in a standardized 3D space (Talairach and Tournoux, 1988). Motion timecourses were obtained by estimating the values for translation (mm) and rotation (deg) for each of the 396 consecutive volumes. Of the 14 measured datasets, 4 second-run datasets were discarded because of head movements larger than $0.8 \mathrm{~mm}$ occurring over several consecutive volumes, as obtained from the motion timecourses. Functional data from individual subjects are depicted on the standardized anatomical volume and on inflated and flattened anatomical representations, following procedures described elsewhere (Kriegeskorte \& Goebel, 2001; Linden et al., 1999). Group data are superimposed on an anatomical brain template (courtesy of the Montreal Neurological Institute).

Functionally connected patterns of activity were estimated using spatial ICA. Formally, the observed data were modelled as:

$$
\mathrm{X}=\mathrm{MS} \text {, }
$$

where $X$ is the $n \times v$ matrix of the observed time courses ( $n=$ number of scans, $v$ $=$ number of voxels), $S$ is the $m \times v$ matrix whose rows $S_{\text {.i }}$ are to be filled with the (unknown) realizations of the spatial components ( $m \leq n=$ number of components), and $M$ is the $\mathrm{n} \times \mathrm{m}$ "mixing" matrix, whose columns contain the time-courses of the m components and is assumed to be of full rank. In this model, all the spatial components, with the possible exception of one, are assumed to be non-gaussian (Hyvärinen, 1999). Structured (non-gaussian) artifacts in the data (e.g. head movements, machine and physiological artifacts) are not explicitly modelled, but instead are treated as independent sources and are thus expected to be represented in one or more of the components. 


\section{Chapter 1}

The problem of the ICA-decomposition of fMRI time-series can be formulated as the estimation of both the matrices of the right side of equation (1.1), under the constraint that the processes $\mathrm{S}_{\mathrm{i}}$ are (in the ideal case) mutually statistically independent. No a priori assumption is made about the mixing matrix $M$, i.e. about the time courses. The amount of statistical dependence within a fixed number of spatial components can be quantified by means of their mutual information (Comon, 1994). Thus, the ICA decomposition of X can. be defined (up to a permutation of the components, a multiplicative constant and to the sign) as a linear transformation:

$$
s=W X
$$

where the matrix $W$ (the "unmixing" matrix) is determined such that the mutual information of the target components $S_{. i}$ is minimized. Matrix $M$ can be computed as the pseudo-inverse of $W$.

Different ICA algorithms use different strategies (see Hyvärinen, 1999 for a review and Esposito et al., 2002 for an empirical comparison of the two ICA algorithms most commonly used in fMRI data analysis). Here, the $4 \mathrm{D}$ functional data sets were decomposed using the cortex-based sICA methodology (cbiCA: Formisano et al., 2001; 2002) that uses a fixed-point ICA algorithm (FastICA, Hyvärinen, 1999) as implemented in www.cis.hut.fi/projects/ica/fastica/. The cortex-based approach restricted the ICA to a subsample of the voxel timecourses belonging to the cortical sheet, thus reducing the data by $65-70 \%$. This approach was justified because we were primarily interested in cortical functional connectivity, without restricting the analysis to specific functionally specialized cortical regions. The FastICA algorithm minimizes the mutual information of the components using a robust approximation of the negentropy as a contrast function and a fast, iterative (non-adlaptive) algorithm for its maximization (for a detailed description of the FastICA algorithm, see Hyvärinen, 1999). After sphering the matrix $X$ and reducing the dimensionality of each data set to 200 components using PCA, the hierarchical (deflation) mode of the FastICA algorithm was used and all the components were estimated oneby-one. The relatively high number of dimensions (200) was selected in order to preserve the original structure of the data (the reduced dataset included more than $99 \%$ of the original variance), as an excessive reduction of the dimensionality may negatively affect the ICA decomposition (McKeown \& Sejnowski, 1998).

After the decomposition, voxel values of the ICA spatial maps were Ztransformed and color-coded according to absolute value and sign (red-yellow: increasing positive Z-values; blue-green: increasing negative Z-values). For visualization the maps were thresholded by $Z$-value $(|Z| \geq 2.2)$, and cluster size ( $\left.>200 \mathrm{~mm}^{3}\right)$, using a three-dimensional 6-neighbor clustering algorithm (Pratt, 1991). It should be noted that the Z-values do not pertain to any significance statistic, since no comparison is made to a null-distribution. Clusters of voxels within a component map that survived the thresholds were 


\section{Functional connectivity during rest}

considered as clusters of functionally connected voxels. After component generation, the component maps were further analyzed in the space and time domains using custom software developed in Matlab 6.x (Mathworks; Inc).

\section{Post-hoc analysis of the components of interest and reproducibility across subjects.}

From the component decomposition of each dataset a subset of components of interest (COIs) was preselected in an automated fashion using only spatial information, as contained within two sets of volumes of interest (VOIs) (see the section on Creation of volumes of interest). Before application of the VOI templates, the component maps were sign corrected. For each component, the mean of the absolute Z-values of the voxels that matched the coordinates of the VOIs was calculated (mean $Z$ in Tables I and II). The absolute, rather than the signed voxel values were used in order to prevent cancellation of signs when averaging the voxel values. The components with the three highest absolute mean-values were selected for further analysis and are referred to as COIs. This criterion was based on consistent findings that component maps with interesting spatial patterns fell within the small range of highest mean voxel values. In addition, each component timecourse was mean-corrected and linearly de-trended, and cross-correlated with the 6 motion timecourses. Components whose time-courses were highly correlated $(r>0.5)$ with the estimated motion time-courses were discarded from further analysis. This second selection criterion was used because we found that in most cases a subsample of the chosen COIs contained contributions from movement artifacts. This second criterion resulted in a further reduction of the number of COIs per VOI by one or two components. The remaining COIs were then used for further analysis.

In order to analyze the contributions of different frequency bands to the spontaneous fMRI activity, a power spectrum analysis of the COI timecourses was performed. COI timecourses were highpass filtered (cut-off $=0.01 \mathrm{~Hz}$ ), and their power spectrum density was estimated by using Welch's method (see Childers, 1987). In particular, we estimated the contribution of the extremely low to low frequencies $(0.01 \mathrm{~Hz}-0.1 \mathrm{~Hz}$ : Cordes et al., 2001) to the overall spectral power, using methods similar to those described in Thomas et al. (2002). We quantified the contribution of low frequencies to the overall power as the ratio between the estimated area under the power spectral curve in the frequency band 0.01-0.1 and the estimated total area. Contribution values range between 0 and 1 , with higher values indicating higher contributions of the low frequency band to the overall power.

The spatial overlap of COIs across subjects was verified using inter-subject consistency maps that were computed on the basis of data from the first measurement of each subject $(N=7)$. In these maps, the voxel value indicates in what proportion of subjects a voxel pertained to the COI in question. For instance, a voxel value of 1 indicates that the $Z$-value at that voxel exceeded the 


\section{Chapter 1}

threshold of $Z=2.2$ in all subjects. The maps were thesholded at a mininum of 3 subjects (map value $>0.29$ ) and were supermposed upon the MNI anatomy template.

Maps were sign corrected across sessions. Mean $Z=$ mean $|Z|$ values of the voxel values captured within the respective VOI template; mean Zsupra $=$ mean $|Z|$ values of voxel values within the VOI template that exceed the $Z$ threshold of 2.2; Nvox In = percent of suprathreshold voxels within the template; Rank = ranking position within the selected subsample of COIs ( $N=$ 3) for each VOI template. Left and right sensory or motor VOI selected the same component, indicating that the component map represented bilateral connectivity patterns, except where indicated with a *

Table 1.1. Detection characteristics of the components of interest for the first volume of interest-set comprising sensory and motor cortices for the first sessions.

\begin{tabular}{|c|c|c|c|c|c|c|c|c|}
\hline \multirow[t]{2}{*}{$\mathrm{VOl}$} & & \multicolumn{7}{|c|}{ subjects } \\
\hline & & 1 & 2 & 3 & 4 & 5 & 6 & 7 \\
\hline \multirow[t]{4}{*}{ Left SMC } & mean $Z$ & 1.87 & 1.86 & 211 & $2.61^{*}$ & - & 1.66 & 4.71 \\
\hline & mean $Z_{\text {swpra }}$ & 3.83 & 3.46 & 3.41 & 4.40 & - & 3.60 & 5.96 \\
\hline & Nwox In & 9.65 & 2.62 & 7.36 & 11.17 & - & 7.13 & 18.77 \\
\hline & Rank & $\sqrt{1}$ & 2 & 1 & 1 & - & 2 & 1 \\
\hline \multirow[t]{4}{*}{ Right SMC } & mean $Z$ & 2.24 & 4.19 & 2.67 & $1.94^{*}$ & - & 223 & 4.65 \\
\hline & mean $Z_{\text {sippra }}$ & 3.61 & 5.16 & 4.35 & 3.78 & - & 3.68 & 5.33 \\
\hline & Nvox In & 12.12 & 18.48 & 10.98 & 8.77 & - & 10.83 & 16.50 \\
\hline & Rank & 1 & 1 & 1 & 1 & - & 1 & 1 \\
\hline \multirow[t]{4}{*}{ Left $\mathrm{AC} 1$} & mean $Z$ & 1.62 & $1,31^{*}$ & 2.64 & 1.16 & $1.14^{*}$ & 1.36 & 1.63 \\
\hline & mean $Z_{\text {supra }}$ & 4.03 & 2.99 & 3.85 & 3.97 & 3.50 & 3.20 & 3.42 \\
\hline & Nvox In & 10.26 & 7.14 & 19.85 & 3.92 & 5.03 & 8.61 & 12.37 \\
\hline & Rank & 1 & 1 & 1 & 2 & 2 & 2 & 1 \\
\hline \multirow[t]{4}{*}{ Left $A C 2$} & mean $Z$ & 1.39 & - & - & - & - & - & $*$ \\
\hline & mean $Z_{\text {sug }}$ & 3.35 & - & - & - & - & " & - \\
\hline & Nvox In & 7.48 & - & - & $=$ & - & - & - \\
\hline & Rark & 3 & - & - & - & - & $=$ & - \\
\hline \multirow[t]{4}{*}{ Right ACl } & mean $\mathrm{Z}$ & " & $1.20^{*}$ & 1.54 & 1.47 & $0.74^{\star}$ & 2.19 & 1.67 \\
\hline & mean $Z_{\text {siuprat }}$ & - & 2.88 & 3.47 & 3.96 & 271 & 4.03 & 3.58 \\
\hline & Nwox In & - & 6.72 & 11,13 & 9.68 & 4.54 & 14,95 & 10.42 \\
\hline & Rank & - & 1 & 1 & 2 & 3 & 1 & 1 \\
\hline \multirow[t]{4}{*}{ Right $\mathrm{AC} 2$} & mean Z & 0.88 & - & - & - & * & - & - \\
\hline & mean $Z_{\text {stppria }}$ & 3.06 & - & - & - & - & - & - \\
\hline & Nvox In & 3.45 & - & - & - & - & - & - \\
\hline & Rank & 3 & $\therefore$ & - & - & $=$ & - & - \\
\hline \multirow[t]{4}{*}{ PVC } & mean $Z$ & 2.90 & 1.18 & 2.82 & 2.82 & 3.18 & 2.95 & 1.63 \\
\hline & mean $Z_{\text {supra }}$ & 4.29 & 3.36 & 4.46 & 5.25 & 4.71 & 4.85 & 4.00 \\
\hline & Nrox In & 41.95 & 11.66 & 50.59 & 32.78 & 45.20 & 51.69 & 23.27 \\
\hline & Rank & 1 & 1 & 1 & 1 & 1 & 1 & 1 \\
\hline
\end{tabular}




\section{Reproducibility within subjects}

For those subjects that had two valid measurements $(\mathrm{N}=3$; subjects 1,4 and 6 ) the reproducibility of the COIs was assessed by verifying the spatial overlap between corresponding COIs obtained in different sessions (spatial correlations between the sign-corrected maps) and by evaluating their cross-spectral density using Welch's method (see Childers, 1978). The similarity of the replicated COIs was also verified on the basis of two component-descriptive parameters: 1) the degree of clustering of suprathreshold voxels (number of clustered voxels / number of suprathreshold voxels) within the component maps, which serves as an index of spatial structure for each component, and 2) the one-lag serial atutocorrelation coefficient of the COI timecourse, which serves as a measure of temporal structure (Formisano et al., 2002). Both measures range from 0 to 1 . It has been shown that COIs tend to possess a sparse and spatially clustered distribution of high Z-values and have an intermediate to high temporal structure, which is reflected in intermediate to high values of these measures (Formisano et al., 2002; Van de Ven et al., 2002).

\section{Creation of volumes of interest}

To preselect COIs on the basis of standardized anatomical or functional information we created two sets of volumes of interest. The first set comprised VOIs of auditory, visual and sensorimotor regions, which are often the regions of interest in resting state functional connectivity analysis. For the auditory cortex VOI a probabilistic mapping of Heschl's gyrus, the putative site of primary auditory cortex, was used (Rademacher et al., 2001). Such maps depict the probability for any anatomical voxel to belong to left or right Heschl's gyrus with respect to an anatomically averaged template. In our study, the probability level of the maps was thresholded at a minimum of $20 \%$ in order to capture the high anatomical and functional variability of the auditory cortices [left centre of mass $(\mathrm{COM}): x=-40, y=-20, z=8$, number of voxels $(\mathrm{NoV})=4910$; right CoM: $x$ $=45, y=-13, z=10, \mathrm{NoV}=4541]$. For the visual cortex VOIs, the area around the calcarine fissure was chosen [CoM: 0, -79, 1, NoV $=10576]$. For the sensorimotor cortex VOIs, regions were chosen using anatomical landmarks (pre- and postcentral gyri) and functional activity clusters from a study that used button presses to identify periods of auditory hallucinations (Van de Ven et al., 2002) [left CoM: $-37,-24,43, \mathrm{NoV}=3732$; right $\mathrm{CoM}: 41,-22,52$, NoV = 5159.

The second set of VOIs was derived from functional and anatomical information, and comprised cortical regions within the parietal lobe: bilateral intraparietal sulcus region (IPS [left CoM: $-30,-60,38, \mathrm{NoV}=6592$; right CoM: $28,-54,45, \mathrm{NoV}=7089])$ and the posterior cingulate cortex/precuneus (pCC/ventral preC [CoM: $-1,-47,24, \mathrm{NoV}=13319]$ ). These regions were used for a post-hoc COI selection to detect parietal resting state networks. The addition of these VOls to the sensory and motor VOIs reflects our interest in 


\section{Chapter 1}

parietal regions, which we investigated in a number of studes of wisuospatal paradigms (e.g. Formisano et al., 2002; Trojano et al, 2000)

Table 1.2. Detection characteristics of the components of interest tor the second volume of interest-set comprising parietal regions for the first sessions.

\begin{tabular}{|c|c|c|c|c|c|c|c|c|}
\hline \multirow[t]{2}{*}{ : $\mathrm{VOI}$} & : & : & & 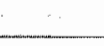 & subjects & $\vdots$ & & \\
\hline & $\cdots$ & 1 & 2 & 3 & 4 & 5 & 6 & 7 \\
\hline \multirow[t]{4}{*}{ Left $\triangle P C$} & $\operatorname{mean} Z$ & $170^{*}$ & $2.66^{*}$ & $1.06^{*}$ & $1.60^{\circ}$ & $0.85^{*}$ & $1.66^{*}$ & $0.98 *$ \\
\hline & mean $Z_{\text {suproia }}$ & 4.48 & 4.30 & 4.26 & 3.83 & 3.28 & 3.88 & 411 \\
\hline & Nvax In & 12.23 & 17.74 & 7.07 & 9.32 & 5.57 & $13.0 \%$ & 5.59 \\
\hline & Rank & 1 & 1 & 2 & 1 & 2 & 1 & 3 \\
\hline \multirow[t]{4}{*}{ Right IPC } & $\operatorname{mean} Z$ & $1.60^{*}$ & $3.37^{*}$ & $1.50^{*}$ & $2.67^{*}$ & $0.94^{\prime \prime}$ & $238^{\mathrm{k}}$ & $1.52^{*}$ \\
\hline & mean $Z_{\text {suppra }}$ & 4.10 & 4.72 & 4.87 & 4.48 & 2.26 & 4.79 & 5.45 \\
\hline & Nvox In & 8.87 & 19.47 & 8.39 & 16.60 & 8.79 & 15.69 & 4.99 \\
\hline & Rank : & 2 & 1 & 2 & 1 & 3 & 1 & 2 \\
\hline \multirow[t]{4}{*}{$p C C$} & $\operatorname{mean} Z$ & 1.92 & 2.33 & 2.03 & 1.32 & 1.65 & 1.70 & 1.69 \\
\hline & mean $Z_{\text {sup }}$ & 4.54 & 4.45 & 4.10 & 3.27 & 4.06 & 3.97 & 4.98 \\
\hline & Nvox In & 26.57 & 28.07 & 24.11 & 16.80 & 20.36 & 23.83 & 23.92 \\
\hline & Rank & 1 & 1 & 1 & 3 & 2 & 1 & 1 \\
\hline \multirow[t]{4}{*}{$\mathrm{pCC} /$ prec1 } & $\operatorname{mean} Z$ & 1.797 & - & -1.254 & -0.376 & 1.733 & 0.760 & 1.127 \\
\hline & mean $Z_{\text {sapra }}$ & 4.716 & - & -3.362 & -0.705 & 3,965 & 2.713 & 3.985 \\
\hline & Nvox In & 24.93 & - & 18.64: & 19.50 & 20.79 & 18.69 & 13.53 \\
\hline & Rank & 2 & - & 3 & 2 & 1 & 2 & 2 \\
\hline \multirow[t]{4}{*}{$\mathrm{pCC} /$ prec2 } & mean $Z$ & - & - & - & 2.33 & 0.67 & - & -0.33 \\
\hline & mean $Z_{\text {supra }}$ & - & $=$ & - & 4.11 & 2.49 & - & 0.70 \\
\hline & Nwox In & - & - & - & 31.23 & 11.41 & - & 12.33 \\
\hline & Rank & $\therefore$ & - & - & 1 & 3 & - & 3 \\
\hline
\end{tabular}

Left and right IPC components also included clusters in dorsolateral prefrontal cortex and frontal eye fields in the same hemisphere. For explanations of abbreviations, see Table 1.1 .

\section{Results}

\section{Volumes of interest of sensory and motor cortex}

For each data set, some of the three selected COIs per VOI template were discarded because they reflected contributions of artifacts (the criteria are described in the Methods section). In most cases the VOI-based preselection using the VOIs of sensory and motor cortices (first VOI set) yielded one $\mathrm{COI}$ connectivity map of the bilateral primary and secondary auditory cortex $(\mathrm{AC})$, one map of bilateral visual cortex (PVC) and one map of bilateral sensorimotor cortex (SMC) (Table 1.1). Selection of the same component by the left and right sensory or motor VOI indicated that the component contained bilaterall 
connectivity pattems. These COls ranked highest in the VOI-based selection (see Table 1.1). In addition, these components were largely free from motion artifacts.

In one subject no left or right COI of the sensorimotor cortex passed the selection criteria. In another subject, the left and right SMC were represented separately by two different COIs. The functional connectivity clusters for the left and right auditory cortex were represented separately by two different COls in two subjects. For the first session of subject 1 , two bilateral auditory cortex components were selected. One COI was more centered upon Heschl's gyrus in both hemispheres, while the other COI incorporated regions in the anterior and posterior superior temporal gyrus. In the second session of subject 1 only one COI for the auditory cortex was found, which was not a spatial summation of the two COIs from the first session. For all subjects, a COI was found with functional connectivity clusters that included the calcarine fissure in both hemispheres. Figure 1.1 shows the COI maps of auditory, visual and sensorimotor regions of one representative subject (subject 6). These connectivity clusters showed a marked consistency of spatial characteristics among the various decompositions, as is reflected by the inter-subject consistency maps in Figure 1.2. Surprisingly, a functional connectivity cluster centered on the middle and lower parts of the central sulcus (CS), was identified by the SMC or the AC VOI in most cases (see Figure 1.2), although we did not explicitly create a VOI for the entire extent of the bilateral central sulcus. The detection of this region of connectivity can be attributed to the fact that SMC and $\mathrm{AC}$ VOI-voxels, respectively, extended into the top or lower end of the CS clusters (see Figure 1.2).

\section{Volumes of interest of parietal areas}

For the second VOI set, the pCC/ventral preC VOI selected one or two COIs comprising connectivity clusters in bilateral $\mathrm{pCC} / \mathrm{preC}$, and one $\mathrm{COI}$ connectivity map comprising preC and bilateral superior parietal lobule (SPL) in the majority of the decompositions (Figures 1.1 and 1.2, Table 1.2). The left and right IPS VOI selected one COI connectivity maps comprising either the left or the right inferior parietal cortex (IPC) from most of the decompositions. The IPC connectivity maps consistently comprised small connectivity clusters in left or right dorsolateral prefrontal cortex (DLPFC) and frontal eye fields (FEF). In all cases the COI for the IPC contained unilateral clusters, suggesting that these regions may not be functionally connected across hemispheres during rest. 

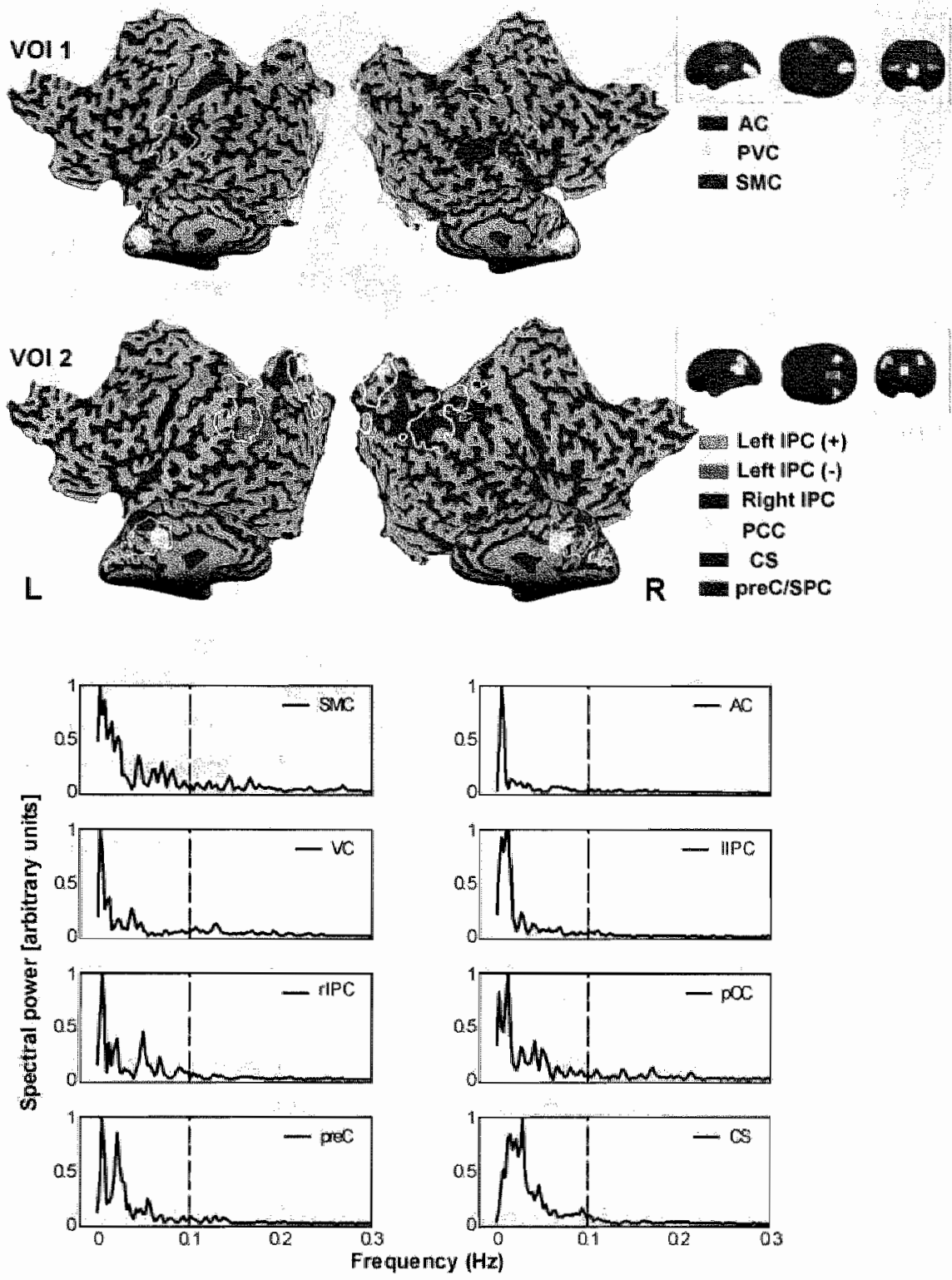

Figure 1.1. Upper panel: Components of interest (COIs) obtained by using the first (VOI 1) and second (VOI 2) set of VOIs of subjects 6 . Colors indicate the different COIs. White lines superimposed on the flatmap indicate borders of the VOI templates. Inserts: the VOI templates of the first and second VOI set are presented in glass brain format. Lower panel: The estimate of power spectral densities of the COI timecourses (normalized according to maximum value of power), showing that the highest powers are found within the low to very low frequency range $(0.01-0.1 \mathrm{~Hz})$. Broken line indicates $0.1 \mathrm{~Hz}$. For explanation of the abbreviations, see abbreviation list. For color version, see page 133 . 

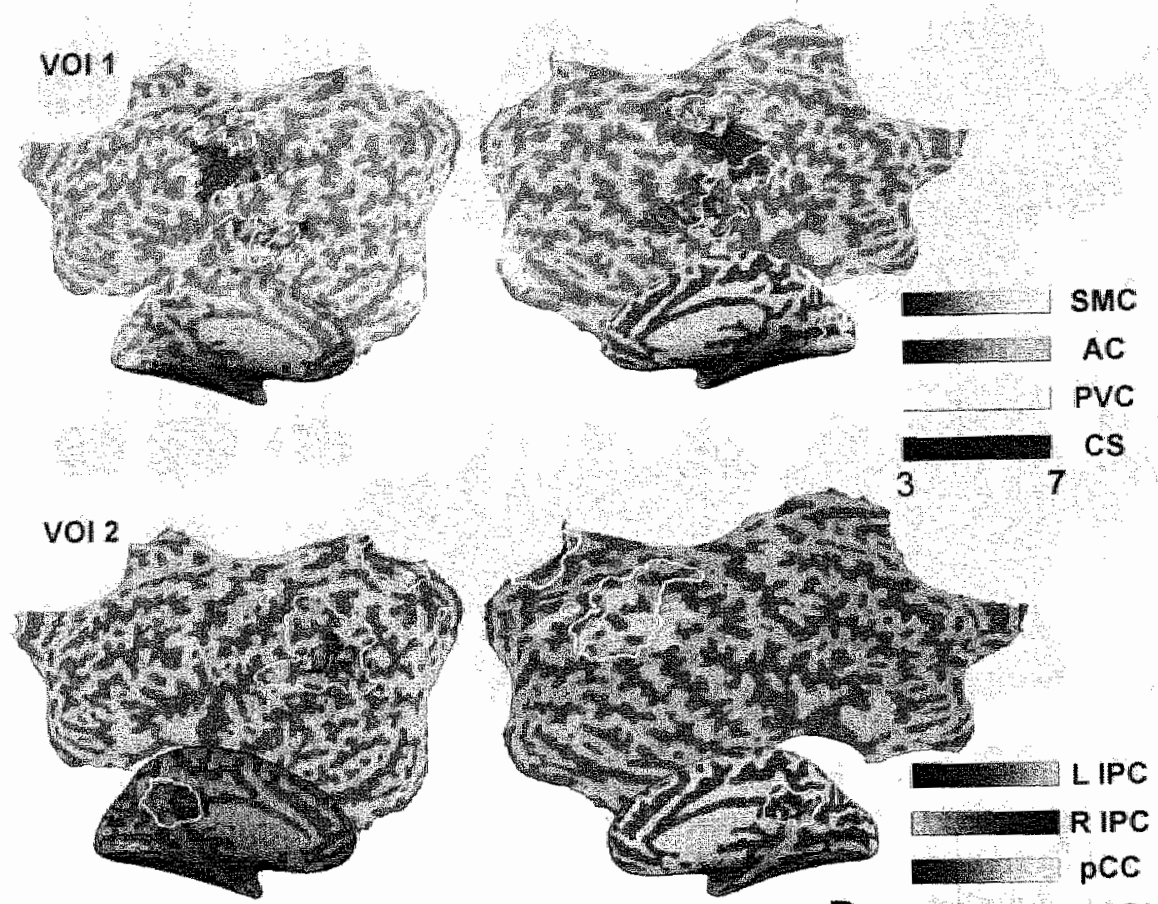

L.

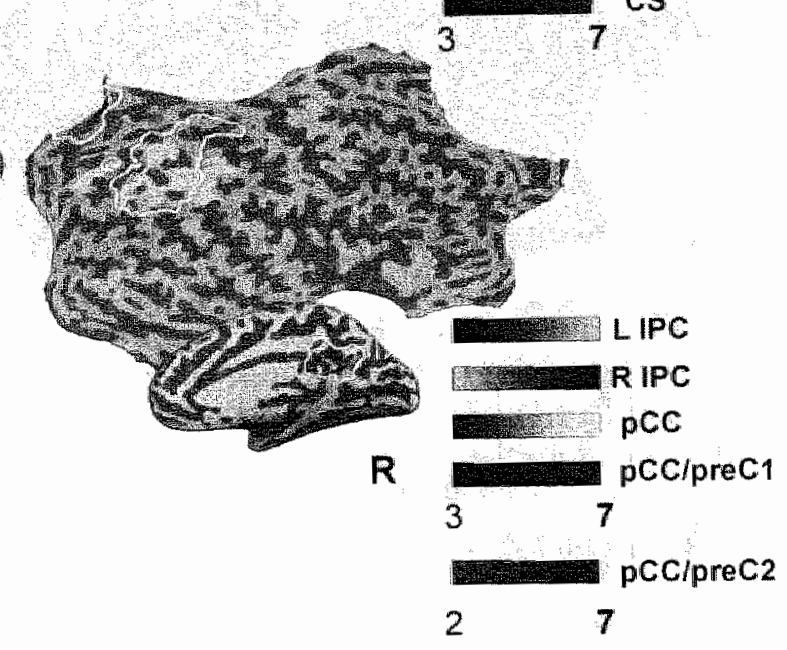

Figure 1.2. Inter-subject consistency maps of the first and second VOI set for the first session of all subjects. Maps are obtained by summing the suprathreshold voxels contained within the respective COIs across subjects, and divided by the total number of subjects. Map values range from 0 to 1 , and are thresholded at $>0.29$. Maps are depicted on MNI template flatmaps. White lines on the fatmap indicate borders of the VOI templates. For color version, see page 134.

\section{Frequency analysis of the component of interest timecourses}

The frequency plots for the COIs containing auditory, sensorimotor and visual regions show that the largest contributions to the frequency spectrum are within the extremely low range $(0.01-0.05 \mathrm{~Hz})$ of the component timecourse. Frequency plots for subject 6 are presented in the lower part of Figure 1.1. For the COIs selected by the second VOI set that comprised parietal regions, higher powers were observable for the higher frequencies. The contribution ratio of the spectral power in the frequency range of $0.01-0.1 \mathrm{~Hz}$ to the whole spectral range was estimated for the COIs of all first sessions. On average, all values exceeded the ratio of 0.4 , which indicates that a large amount of the spectral 


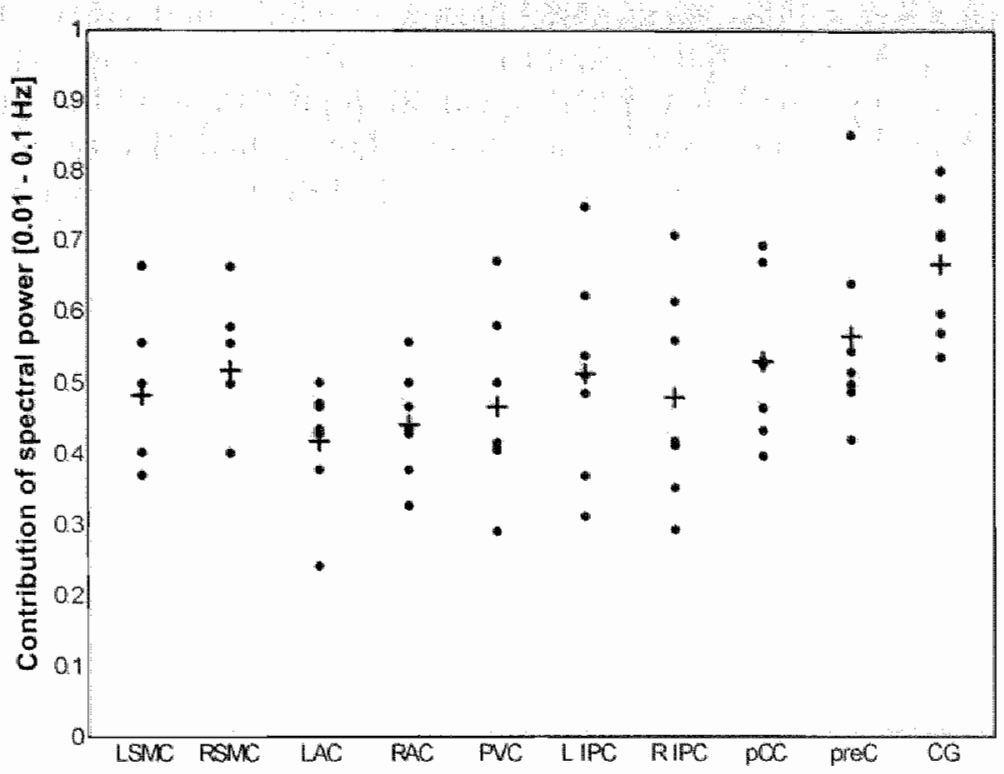

Figure 1.3. Dispersion plot. Contribution ratio of the spectral power within the low to very low frequency range $(0.01-0.1 \mathrm{~Hz})$ for the COls for the sessions of all subjects (dots), and the average contribution for each COI (4). L-/RSMC = left/right sensorimotor cortex; $\mathbb{L}-/ \mathrm{RAC}=$ left-/right auditory cortex; $\mathrm{L}-/ \mathrm{RIPC}=$ left-/right inferior parietal cortex.

power is explained by the frequency powers of the low to very low range (Figure 1.3).

\section{Reproducibility}

In a post-hoc investigation we looked at the reproducibility of the COIs in spatial, temporal and frequency domains within the same subject $\left(\mathrm{N}_{\text {subjer }}=3\right.$; subjects 1,4 and 6). COls as identified by the sensory-motor and parietal VOI sets showed a high degree of spatial consistency across the two sessions of all three subjects (Table 1.3). The upper part of Figure 1.4 depicts this consistency for subject 1 . The lower part of Figure 1.4 shows the consistency in frequency contributions of the COI timecourses across the two sessions of the same subject in the form of cross-spectrum densities. This overlap in spectral density is found for most of the reproduced COIs from the first and second session, which is reflected by the large contribution of extremely low frequencies to the COIs from the first VOI set $(0.01-0.05 \mathrm{~Hz})$, and low frequencies to the COIs from the second VOI set $(0.05 \mathrm{~Hz}$ and higher) (Table 1.4). The similarity of the first and second session COIs was also found in component-descriptive parameters (degree of clustering of suprathreshold voxels and temporal structure of the COI timecourse), as is depicted in Figure 1.5. The pairs of COIs pertaining to 


\section{Functional connectivity during rest}

similar anatomical regions can be found in close proximity in the plane defined by these component-descriptive parameters. In addition, the component pairs can be consistently found in a specific quadrant (upper-right) of the 2-D plot, indicating that these components consistently show a high degree of spatial clustering of functionally connected voxels and that the timecourses are highly structured.

Table 1.3. Intra-subject spatial correlations between the Z-scored sign-corrected spatial COI maps of session 1 and 2 of subjects \#1, 4 and 6 .

\begin{tabular}{|c|c|c|c|c|c|c|c|c|}
\hline \multirow[t]{2}{*}{ Subject } & \multirow[b]{2}{*}{ bill $A C$} & \multirow[b]{2}{*}{ bilsMC } & \multirow[b]{2}{*}{ PVC } & \multicolumn{2}{|c|}{ COIs } & \multirow[b]{2}{*}{$p \subset C$} & \multirow[b]{2}{*}{ prec } & \multirow[b]{2}{*}{$\mathrm{CS}$} \\
\hline & & & & left $\mathbb{I P C}$ & right IPC & & & \\
\hline \#1 & $0.45^{\mathrm{i}}$ & 0.62 & 0.75 & 0.70 & 0.52 & 0.64 & 0.79 & 0.57 \\
\hline \#4: & 0.35 & 0.25 & 0.67 & 0.54 & 0.62 & 0.26 & 0.80 & 0.48 \\
\hline$\$ 6$ & 0.26 & $0.21 \%$ & 0.69 & 0.75 & 0.83 & 0.79 & 0.71 & 0.66 \\
\hline
\end{tabular}

All correlation walues are highly significant $(p<0.0001)$; bil = bilateral. Note: a When in one of the sessions the left and right regions of interest were represented by two separate components, the sign-corrected COls were averaged into a single map.

\section{Discussion}

In this study we analyzed functional connectivity from resting state datasets with spatial cortex-based independent component analysis. Using a volume of interest-approach (VOI-approach) to automatically preselect a small number of components for further analysis, we found independent components of interest (COIs) for bilateral auditory, sensorimotor and visual regions, as well as several parietal (preC, SPC, IPC) regions. These components indicated a high degree of functional connectivity between homologous areas in the two hemispheres, presumably subserved by trans-callosal connections. However, the left and right IPC regions showed a consistent decoupling between hemispheres, while they were strongly connected to frontal regions (DLPFC/FEF) of the same hemisphere. Such a pattern has been reported in task-related studies as well (Formisano and Goebel, 2003), thus suggesting that parietal homologous areas do not necessarily subserve homologous functions. Unexpectedly, for each subject ICA revealed a component map showing bilateral central sulcus connectivity stretching from motor cortices to inferior frontal gyri. Frequencypower analysis of the COI-timecourses showed that extremely low frequencies $(0.01-0.05 \mathrm{~Hz})$ contributed largely to the frequency spectrum. Spatiotemporal decomposition and selection of the COIs proved to be highly consistent across as well as within subjects.

Our results are similar to previously reported functional connectivity maps of auditory, visual and motor cortices in space (Biswal et al., 1997; Lowe et al., 1998; Cordes et al., 2001) as well as in time (Cordes et al., 2001; Xiong et al., 


\section{Chapter 1}

1999). However, our spatial ICA application yielded onlly one component for each region of interest in the majority of cases, while Kiviniemi et al: (2003) reported multiple components for sensory and motor regions. This difference may reflect the different samples (young patients ws. healthy adults) and measurement situations (anaesthetized vs. awake) of the two studies. In addition, we used an automated component selection procedure that yielded only one, in some cases two COIs with the highest average absolute Z-values, instead of selection by visual inspection of target areas. The spatial location and extent of our sICA-derived connectivity maps of parietal areas show a high similarity to clusters of activity elicited by several visuospatial tasks, using both hypothesis- (e.g. Goebel et al, 1998; Trojano et al., 2000) and data-driven analyses (Formisano \& Goebel, 2003).

The contribution of low and very low frequencies to the $\mathrm{CO}$ timecourses suggests that the COIs reliably represent the functional connectivity patterns in the time domain as well. Functional connectivity as measured by multi-unit cell recording is often associated with frequencies in the gamma range, and can show high coherence over short and long cortical distances (Fries et al., 2001; Leopold et al., 2003). Yet it has been demonstrated that fluctuations with a frequency range similar to that observed in our COI timecourses $(<0.1 \mathrm{~Hz})$ over a very large time-scale (minutes) are a meaningful indicator of coherent fluctuations of higher frequencies (e.g. gamma range) that occur over a very short time-scale (milliseconds) (Leopold et al., 2003). Functional connectivity maps as measured by fMRI might thus be a good estimator of large scale functional connectivity at the neural level. However, connectivity in the BOLD signal maps may also represent couplings in blood flow or capillary processes. The low frequencies reported in our as well as in other studies have been linked to oscillations of cerebral blood flow regulation (Mitra et al., 1997), which have been found in frequency ranges as low as $0.04 \mathrm{~Hz}$ (Obrig et al., 2000). Our current approach cannot convey any information about the direction of such connectivity patterns because the analyses are based only on zero-lag covariation over time. In addition, fMRI functional connectivity is limited by a coarse temporal resolution (i.e., in the range of seconds), while at the same time subcortical activity (e.g. thalamic activity patterns) is explicitly neglected in the cortex-based analysis. These constraints clearly prevent an interpretation in the context of possible biochemical or neurophysiological modulators of such connectivity patterns.

Spatial ICA does not rely on a predefined temporal model, and is therefore very suitable for functional connectivity analysis of resting state data, where a general model of ideal brain activity cannot be generated. Although several parameters of the dataset may vary within and between subjects (e.g. head movement, anatomical variation), VOI-cbICA was able to reproduce functional connectivity maps of sensory, motor and parieto-frontal regions in the majority of cases. Even more so, comparison of component descriptive parameters such as spatial layout of the functional clusters, frequency spectrum and temporal structure of the COI timecourses yields a high consistency between COls 


\section{Functional connectivity during rest}

obtained from different datasets. This is especially interesting in light of a number of earlier studies where COIs related to acoustic stimulation (Van de Ven et al, 2002), vistual stimulation and motor performance (Formisano et al., 2002) were extracted from different tasks and measurements. The identified COIs showed a high degree of similarity in several component-descriptive parameters across the various studies; as well.
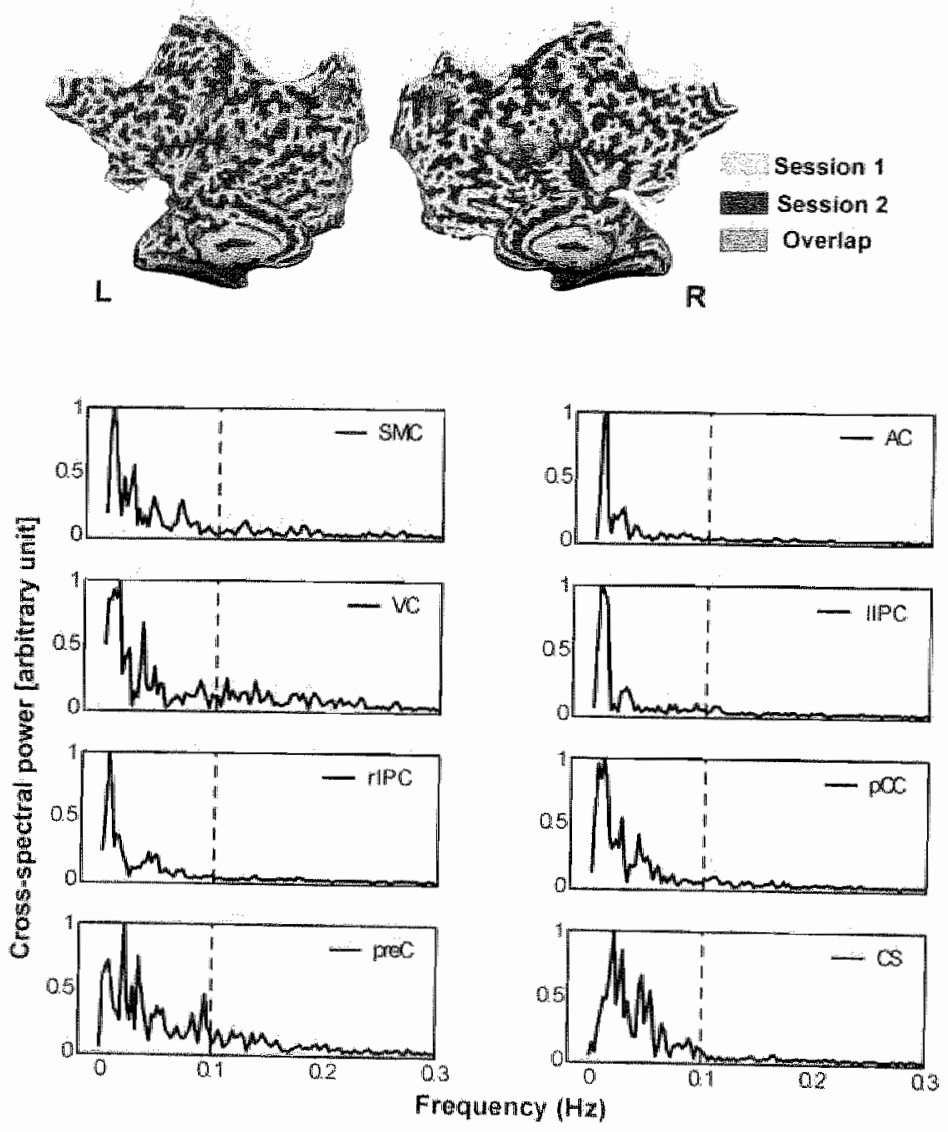

Figure 1.4. (Upper part) Spatiotemporal replication of COI functional connectivity maps of session 1 (blue) and 2 (red) of subject 1. Overlapping regions are depicted in purple. For reference of the anatomical-functional clusters, see Figures 1 and 2 . (Lower part) Cross-spectral densities for the replicated COIs of subject 1 are given for each of the paired COIs (normalized for maximum value). A high spectral density indicates a high power for that frequency in both COI timecourses. For color version, see page 134. 


\section{Chapter 1}

Table 1.4. Contribution ratio of spectral power within $0.01-0.1 \mathrm{~Hz}$ upon the total spectral range of similar components of interest in the two sessions (S1 and S2) of subjects 1,4 and 6 .

\begin{tabular}{|c|c|c|c|c|c|c|c|}
\hline \multirow[t]{2}{*}{ VOI } & 1 & & 4 & & 6 & \multirow[b]{2}{*}{$\mathrm{s} 2$} & \multirow[t]{2}{*}{ Mean } \\
\hline & S1 & S2 & 51 & $\$ 2$ & $\mathrm{S1}$ & & \\
\hline Left SMC & 0.40 & 0.36 & 0.50 & 0.25 & 0.40 & 0.61 & 0.42 \\
\hline Right SMC & 0.40 & 0.36 & 0.50 & 0.25 & 0.40 & 0.49 & 0.40 \\
\hline Left $A C$ & 0.50 & 0.35 & 0.24 & 0.55 & 0.44 & 0.35 & 0.41 \\
\hline Right $A C$ & 0.50 & 0.35 & 0.56 & 0.55 & 0.44 & 0.59 & 0.50 \\
\hline PVC & 0.29 & 0.38 & 0.58 & 0.32 & 0.67 & 0.75 & 0.50 \\
\hline Left IPC & 0.62 & 0.37 & 0.49 & 0.57 & 0.54 & 0.45 & 0.51 \\
\hline Right IPC & 0.35 & 0.51 & 0.29 & 0.49 & 0.71 & 0.38 & 0,46 \\
\hline $\mathrm{pCC}$ & 0.40 & 0.45 & 0.67 & 0.39 & 0.53 & 0.49 & 0.49 \\
\hline $\mathrm{pCC} /$ preC & 0.52 & 0.58 & 0.85 & 0.41 & 0.42 & 0.49 & 0.54 \\
\hline$C G$ & 0.76 & 0.79 & 0.71 & 0.59 & 0.80 & 0.83 & 0.75 \\
\hline
\end{tabular}

For abbreviations, see abbreviations list. $\mathrm{a}=\| \mathrm{eft} / \mathrm{right}$ auditory cortex $\mathrm{COI}$ of subject 1 , session 1 (S1) is left/right auditory cortex (AC1) in Table 1.1.

$\mathrm{CbICA}$ is able to separate functionally comected networks from motionrelated artifacts and other BOLD-signal pattems not related to neural activity. ICA as a noise-reducer has been reported in the literature (McKeown et all, 1998b; McKeown \& Sejnowski, 1998; Thomas et al., 2002), but was mainly aimed at separating noise-related timecourses from task activity related to block or event-related designs. In our study of resting state measurements, noise reduction is an important part of the analysis, since no pre-specified hypothesis can be used to estimate and separate interesting BOLD signals from potentially noise-related activity patterns (McKeown et al., 1998b; Thomas et al., 2002).

Arfanakis et al. (2000) used ICA to identify and remove task-related activity from functional datasets. The resulting data matrix only contained non task-related activity that comprised the baseline conditions and noisecomponents. Functional connectivity networks in sensory and motor regions were then identified with a seed-voxel approach. However, the task-related activity patterns were identified on the basis of a cross-correlation between the stimulus protocol and the component timecourses. Extracting independent components by relying on the stimulus protocol may ignore possible taskdependent activity patterns that are not obviously captured by the shape of the protocol. Such a method may then leave traces of task-dependent activity in the subvolume of the "resting state" data set, resulting in task-dependent connectivity. Our approach functions independently of timecourse information, while it emphasizes spatial information as a criterion for selection of COIs. 


\section{Functional connectivity during rest}

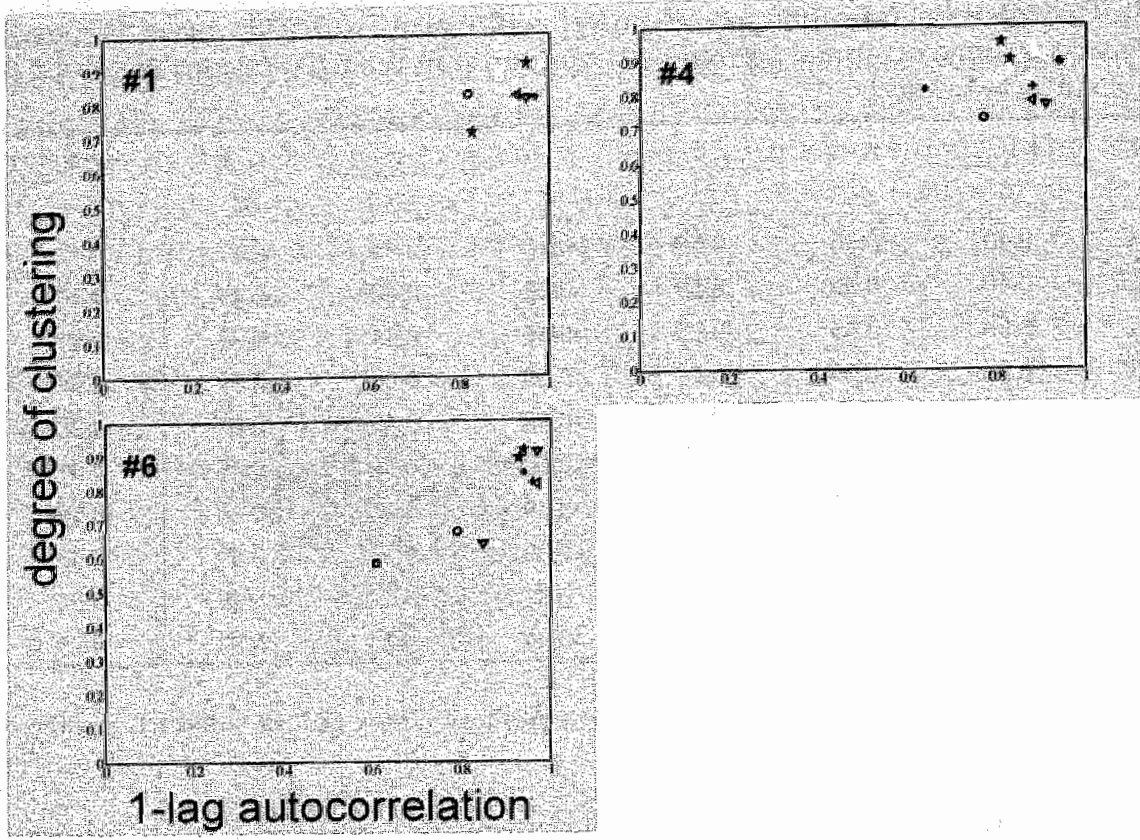

Figure 1.5. Scatter of the COIs of the first (white) and second (black) sessions according to a spatial (degree of clustering) and a temporal (absolute values of the one-lag autocorrelation coefficient) measure. From up to down the plots are of subject 1 ,

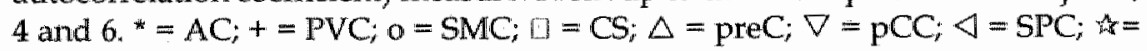
IPC/DLPFC.

The finding of $\mathrm{pCC} / \mathrm{preC}$ functional connectivity COIs is of special interest in the light of recent findings on default states of brain activity in these and other regions. A decrease in the BOLD signal in the ventral anterior and posterior cingulate cortex during task performance in comparison to episodes of baseline activity has been reported by Raichle et al. (2001). This relationship was investigated by Greicius et al. (2003), who looked at activity in these regions during task execution and during resting state measurements. Not only did they find that these regions were functionally connected in the resting state, but that activity in these regions was inversely correlated with activity in prefrontal regions involved in working memory. "This finding is supportive of a "default" brain state of activity that is disrupted when a task is performed (Raichle et al, 2001). However, the COI functional connectivity maps obtained in our study that comprised the $\mathrm{pCC} / \mathrm{preC}$ did not contain clusters in the anterior cingulate cortex. This may suggest that the reported correlations between the regions do not describe a statistically dependent relation. This can be the case when a third region modulates the activity of the regions contained in the COI.

This study has several limitations. First, we chose to decompose each dataset using a relatively large number of independent components. An 
excessive reduction of the dimensionality may be particularly problematic for the analysis of resting state measurements because the interesting sources are expected to be "weak" compared to other, artifactual sources (Green \& Cordes, 2002). Conversely, setting the number of components too high may result in functionally connected regions split into separate components. For example, in one case the bilateral auditory cortex was represented in two components, while the left and right IPC were each captured in a single component map in all cases. However, in most cases the sensory and motor connectivity maps showed a bilateral pattern similar to connectivity patterns reported in other studies, indicating that our choice of the number of components did not play a major role in determining our results. Interestingly, in another resting state study using ICA in which a relatively small amount of components were estimated, multiple connectivity maps for sensory and motor areas were found (Kiviniemi et al., 2003). We conclude that further research on the optimum number of components for decomposition of AMRI data is needed. Second, we used an ICA algorithm that assumes that the timecourses of cortical areas within one component are synchronous, which will result in the representation of areas with timecourses that differ only in latency in separate components (Calhoun et al., 2003). However, this might be useful for studying, e.g., mental chronometry when decompositions can be associated with different processing stages related to task performance (Formisano \& Goebel, 2003). Third, we did not assess subjects' compliance with the explicit instruction to keep their eyes open during the measurement that was performed in complete darkness. Assessment or control of eye movements during resting state measurements may become more important when psychiatric patients are involved, and can be done by using eye movement tracking devices that are scanner-compatible.

Current models of functional and effective connectivity in the brain need to be supplemented by the investigation of direct or indirect anatomical connections between functionally connected cortical areas. Recent developments in the field of diffusion tensor imaging (DTI) may allow for the estimation of trajectories of white matter fiber tracts in the brain. One recent study combined seed-voxel based assessment of functional connectivity in the normal human resting brain with DTI, and reported high functional connectivity for spatially distinct areas that were connected through white matter tracts (Koch et al., 2002). However, correlations between the temporal patterns of spatially distant areas remained high even when direct anatomical connections were lacking, showing that the relation between anatomical and functional connectivity is not straightforward.

A disrupted or otherwise altered pattern of communication within and between specialized brain areas or networks has been suggested to be involved in a number of neurological and psychiatric disorders. Such alterations may result in a default brain state that differs from the putative default state in healthy individuals. For instance, a reduced inter-hemispheric resting state connectivity based on BOLD measurements has been shown in multiple sclerosis patients (Lowe et al., 2002), while reduced functional and effective 
connectivity might be related to symptoms of schizophrenia (Friston, 1998; Hoffman and McGlashan, 1993). Analysis of the connectivity patterns of the resting or "default" brain of these and other disorders is likely to provide useful insights into their underlying pathologies (Calhoun et al, 2004). Moreover, such analysis may supplement FMRI studies that use cognitive paradigms that typically comprise alternating periods of task and rest, which is used as baseline. The use of resting state brain activity as a baseline has been heavily debated (Gusnard \& Raichle, 2001; Stark \& Squire, 2001). In this context, a better understanding of the patterns of resting state activity should lead to an improved interpretation of task-or stimulus-related activation in fMRI studies.

\section{Acknowledgements}

The authors would like to acknowledge Prof. F.E. Zanella and Dr. H. Lanfermann at the Department of Neuroradiology, Frankfurt University, for access to the MR tomograph, and Prof. K. Maurer for constant support. F. de Martino is gratefully acknowledged for his assistance in the analysis. C. Morawetz kindly provided help with one of the figures. Prof. W. Singer and three anonymous reviewers provided very helpful comments on an earlier version of the manuscript. This study was supported by the Alzheimer Forschung Initiative. 


\section{Chapter 2}

\section{The spatiotemporal pattern of auditory cortical responses during verbal hallucinations}

Based on publication:

Van de Ven, V.G., Formisano, E., Röder, C.H., Prvulovic, D., Bittner, R.A., Dietz, M.G., Hubl, D., Dierks, T., Federspiel, A., Esposito, F., Di Salle, F., Jansma, B., Goebel, R. \& Linden, D.E.J. (2005). The spatiotemporal pattern of auditory cortical responses during verball hallucinations. Neuroimage, 27, 644-655. 


\section{Abstract}

Functional magnetic resonance imaging (fMRI) studies can provide insight into the neural correlates of hallucinations. Commonly, such studies require selfreports about the timing of the hallucination events. While many studies have found activity in higher-order sensory cortical areas, only a few have demonstrated activity of the primary auditory cortex during auditory verbal hallucinations. In this case, using self-reports as a model of brain activity may not be sensitive enough to capture all neurophysiological signals related to hallucinations. We used spatial independent component analysis (sICA) to extract the activity patterns associated with auditory verbal hallucinations in six schizophrenia patients. SICA decomposes the functional dataset into a set of spatial maps without the use of any input function. The resulting activity patterns from auditory and sensorimotor components were further analysed in a single-subject fashion using a visualization tool that allows for easy inspection of the variability of regional brain responses. We found bilateral auditory cortex activity, including Heschl's gyrus, during hallucinations of one patient, and unilateral auditory cortex activity in two more patients. The associated timecourses showed a large variability in the shape, amplitude, and time of onset relative to the self-reports. However, the average of the timecourses during hallucinations showed a clear association with this clinical phenomenon. We suggest that detection of this activity may be facilitated by examining hallucination epochs of sufficient length, in combination with a data-driven approach. 


\section{Introduction}

Auditory verbal hallucinations (AVH), the perception of voices without the presence of an external correlate, represent one of the most baffling symptoms of psychiatric disorders, and are a core symptom in the diagnosis of schizophrenia (American Psychiatric Association, 1994). Functional neuroimaging has made major contributions to the understanding of brain activity that underlies hallucinations. Modality- and content-specific brain activity has been observed in higher-order sensory areas of auditory (Silbersweig et al., 1995) as well as visual hallucinations (Ffytche et al., 1998), and early sensory areas have also been implicated in the experience of hallucinations (Woodruff et al., 1997). In particular, the primary auditory cortex (PAC) of the dominant hemisphere was found to be active during AVH (Dierks et al., 1999), and the lateralisation of the activity suggests the involvement of language-related processes in AVH (Stephane et al., 2001), which is furthermore supported by alterations in white matter fibre tracts connecting languagerelated areas in hallucinating schizophrenia patients (Hubl et al., 2004). Activity in $\mathrm{PAC}$ is not found during the internal generation of an auditory image of spoken text (Shergill et al., 2001) or music (Halpern \& Zatorre, 1999). Mental imagery is typically distinguished from the experience of hallucinations in terms of vividness and degree of control that one can exert upon the percept. The functional dissociation of activity patterns in the early auditory perception system between hallucinations and auditory mental imagery might thus be reflected in the increased vividness and lack of subjective control of $\mathrm{AVH}$.

The finding of PAC activity during $\mathrm{AVH}$, however, has remained controversial (Shergill et al., 2000a), with only few replications (Bentaleb et al., 2002; Lennox et al., 2000). It has been argued that the measurement design used in these FMRI studies may have been a confounding factor that produced the PAC activity during hallucinations (Shergill et al., 2000a). Most fMRI studies apply a functional measurement sequence that has short delays between consecutive whole-brain measurements. Thus, the broadband sound of the EPI sequence may function as an acoustic mask for the ongoing activity in the auditory cortex (Bandettini et al., 1998), which may influence the activity of $P A C$ in a transient, unexpected manner. At the same time, the overt signalling of the timing of the hallucinations may confound the analysis. To counteract these methodological issues, Shergill et al. (2000a) used a design where singlevolume measurements were separated by time-intervals ranging between 30 and 60 seconds (random sampling design), and where the patients reported on their hallucinations after each measurement. The authors did not find PAC activity associated with hallucinations, and argued that the PAC activity found by others may have been modulated by scanner noise or hallucination-related self-reports. However, the potential benefit of the random sampling design is offset by a loss of temporal resolution and sensitivity. 


\section{Auditory cortical response during $\mathrm{AVH}$}

Previous studies of hallucinations relied on correlation analysis (Bandettini et al., 1993) to identify signals of interest, which typically implies that a sequence of self-reports serves as a model for brain activity, which is applied to the dataset in a voxel-per-voxel manner. This model, however, may not be ideal to trace brain activity related to hallucinations. For example, some cortical areas may show an onset of activity that precedes the button press by several seconds (Ffytche et al., 1998; Lennox et al., 1999). Moreover, the neural signatures of hallucinations may exhibit a much more complex variability than the responses to externally presented stimuli in experimental paradigms, for which a precise input function can be defined. At the same time, potential relations between the voxel timecourses (e.g., functional connectivity elements in the data) are ignored.

In the current study we used spatial independent component analysis (sICA: McKeown et al., 1998a) to investigate the cortical activity patterns during AVH. SICA is a multivariate, data-driven method that does not rely on a predefined model of brain activity. Instead, sICA estimates a set of spatial source components from the observed data, which are characterised by maximum spatial independence between components (McKeown et al., 1998b). For neurophysiologically interesting signals, high voxel values tend to cluster in space (Formisano et al., 2002; Van de Ven et al., 2004). This approach has proven to be useful in separating spatial networks of activity that are related to neurophysiological events of interest from activity patterns that are related to other sources, such as head movements. SICA is able to separate networks of activity that are related to the random presentation of acoustic stimuli of varying duration (Gu et al., 2001), which makes it very suitable for the analysis of hallucinations. At the same time, sICA has been used to characterize deviating patterns of functional coupling in auditory cortex in schizophrenia (Calhoun et al., 2004). The temporal variability of the activity patterns of the obtained spatial maps can be further analysed using the BOLD-image plot (Duann et al., 2002). This visualization tool allows for the investigation of single trial responses and aids the further interpretation of the extracted activity
patterns.

We investigated activity in primary and secondary auditory cortex during AVH in schizophrenia using SICA and BOLD-image plots. Data of the same patients listening to acoustic stimuli were analysed in the same way as the hallucination data. We demonstrate hallucination-related activity in auditory cortex, including Heschl's gyrus, in a number of patients. A large variability of the shape and timing of the cortical response during hallucinations compromises its detection by conventional correlation analysis, in which case
sICA can complement the analysis. 


\section{Methods}

\section{Subjects}

Six paranoid schizophrenia patients were recruited from the participating hospitals. Table 2.1 lists the age, sex and medication of each patient at the time of measurement. Diagnosis was based on DSM-IV criteria (American Psychiatric Association, 1994), and was assessed by the treating psychiatrists. All patients were right-handed. The patients suffered predominantly from auditory verbal hallucinations that consisted of running commentary with derogatory content and that were resistent to antipsychotic medication. After the aim and instruction of the study had been explained to the patients, written informed consent was obtained. The study was approved by the local ethics committees.

\section{Design}

The data were acquired in the course of two separate studies on auditory hallucinations, one of which was published previously (Dierks et al., 1999). Patients A, B and C participated in a follow-up of this study (study 1). Patients $D, E$ and $F$ participated in a second study (study 2), which differed from study 1 by using only tones to localize PAC, and by acquiring the functional data with a higher temporal resolution.

For each patient the measurements comprised two functional (one for auditory hallucinations and one for acoustic stimulation), and one anatomical measurement. During the hallucination measurement the patient was required to press a button upon the onset of an auditory hallucination, hold it pressed during the hallucination, and release the button when the hallucination subsided. The sequences of button presses were converted into a reference timecourse for correlation analysis by assigning the value one to each volume in which the patient had pressed the button and zero to all other volumes. The reference timecourse was corrected for the delayed hemodynamic response (Boynton et al., 1996). Acoustic stimulation measurements were conducted in order to functionally localize the auditory cortex in each individual patient. During the acoustic stimulation measurement the patient was required to passively listen to the presented stimuli, using a custom made, scannercompatible acoustic presentation system. For the patients of study 1 the acoustic stimuli consisted of blocks of speech, reversed speech and tones $(2 \mathrm{kHz})$, lasted for $24 \mathrm{~s}$ ( 8 volumes) each, and where interspersed by blocks of rest of equal length. For the patients of study 2 acoustic stimuli comprised blocks of tones of $0.5,1,2$ and $4 \mathrm{kHz}$. Patients $\mathrm{D}$ and $\mathrm{F}$ were presented with blocks of tones that lasted for $20 \mathrm{~s}$ each ( 10 volumes) and were interspersed by blocks of rest of equal length, during which no stimuli were presented. For patient $\mathrm{E}$, the blocks of tones and rest had variable lengths. Reference timecourses for acoustic stimulation were obtained in the same way as for the hallucination epochs. 
Auditory cortical response during AVH

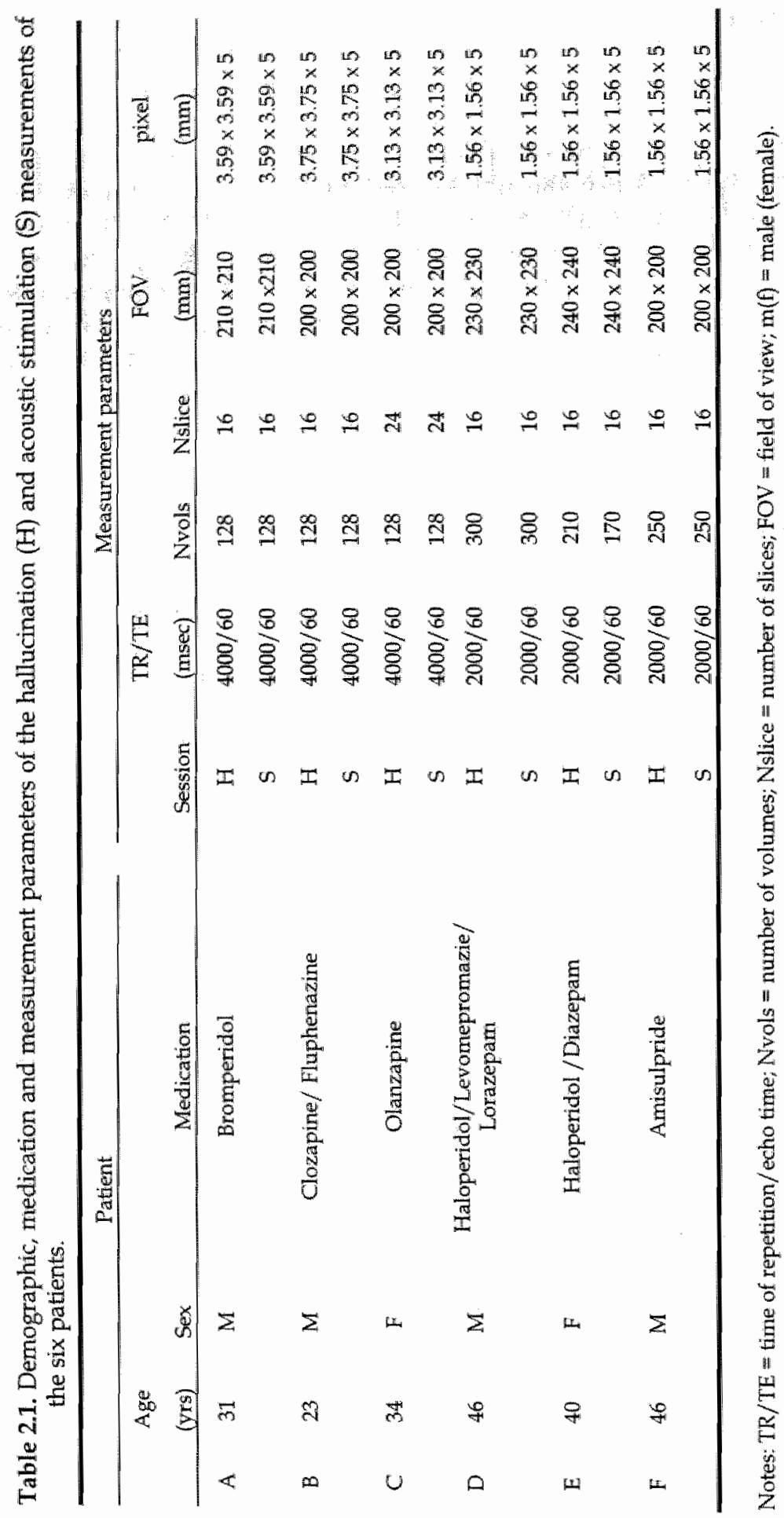


All functional and anatomical images were acquired using a $1.5 \mathrm{~T}$ Magnetom Vision (Siemens, Erlangen, Germany) whole-body tomograph, using a gradient-echo EPI sequence for the measurement of the blood-oxygen-level dependent (BOLD) signal. Table 2.1 lists the imaging parameters for the different sessions. For each patient a T1-weighted anatomical volume (MPRAGE: magnetization-prepared rapid acquisition gradient echo) was acquired (voxel size $=1 \mathrm{~mm}^{3} ;$ matrix $=256 \times 256 \mathrm{~mm}$ ), which lasted approximately 8 minutes.

\section{Analysis}

\section{Preprocessing and independent component analysis}

Preprocessing spatial standardization and SICA of the images were performed using the BrainVoyager 4.8 software (www.brainvoyager.com). Preprocessing of the functional images included slice time correction and 3-dimensional spatial standardization into the Talairach and Tournoux (1988) coordinate space with a resampled functional voxel size of $3 \times 3 \times 3 \mathrm{~mm}$. The timecourses were then temporally filtered by removing linear trends and by high-pass filtering of $1 / 125 \mathrm{~Hz}$ per functional run. The individual anatomical datasets were used to create a cortical mask that tags the corresponding functional voxels, which were then used for further analysis (Formisano et al., 2004).

The functional datasets were individually analysed using cortex-based spatial independent component analysis (Formisano et al., 2002; 2004), which uses the FastICA algorithm (Hyvärinen 1999). The application of cortex-based sICA to fMRI data is described in detail elsewhere (Formisano et al., 2004). Briefly, ICA in its general form models the observed data as a linear mixture of a set of statistically independent (latent) source variables, the number of which is equal or less than the number of observed variables. The size of the decomposition can be set maximally to the number of observed variables, where the reduction is often achieved using principal component analysis (McKeown et al., 1998a,b). The formal descriptions of the ICA model and the decomposition of the observed data into independent components are given by equations (1.1) and (1.2) (chapter 1 of this thesis). In ICA, both the mixing matrix and the source matrix are initially unknown, and component estimation is commonly achieved by maximising the statistical independence between the latent variables, which can be done using different algorithms and estimates for statistical independence (Hyvärinen, 1999). FastICA maximizes statistical independence by maximising negentropy, which is a measure of nongaussianity of the probability distribution (Hyvärinen, 1999). The independent components are modelled as spatially independent, and are therefore commonly referred to as component maps. The voxel values of the component maps (the rows of $S$ in equation (1.2)) are the component weights, and the voxel weight of one component conveys no information about the voxel values of any 
other component map, thereby maximizing spatial independence. The component maps are assumed to be time-invariant, i.e., the layout of the component maps is assumed to be the same during the whole measurement. The contribution of an independent component to the data may be different for each observed variable, and plotting the contribution of a component map to the consecutive volumes constitutes the component timecourse, as contained within the columns of $W$ in equation (1.2).

Selecting the number of components that must be estimated from the data is an important but complicated issue. Different heuristics for setting the number of components for the decomposition have been used (e.g., retaining $99 \%$ to $100 \%$ of the variance of the initial data [Gu et al., 2001; McKeown et al, 1998a]). More formal methods for choosing the number of components have been suggested in the literature (Calhoun et al,; 2001a,b). In this study, we used a series of decompositions with different numbers of components in order to assess the reliability of components of interest. The initial decomposition was set at $1 / 4$ times the number of volumes of the functional dataset for each individual measurement (this number was chosen arbritrarily, while keeping the number of components relatively low and retaining more than $99 \%$ of the variance). Two additional decompositions were performed afterwards, one with $1 / 3$ of the number of volumes as number of components, and one with 100 components for each dataset, regardless of the number of volumes. The reduction of the initial dimension of the dataset to the size of the decomposition was achieved through principal component analysis.

For visualization the voxel values of each component map were $Z$-scored and thresholded at an absolute Z-value of 3 and a cluster size of 6 suprathreshold functional voxels. The maps were then superimposed on the anatomical image.

\section{Selecting components of interest and BOLD-image plots}

Selection and analysis of components of interest (COI) was performed using custom software programmed in Matlab 6 (MathWorks, Inc.). In this study we focussed only on the auditory cortex and sensorimotor cortex contralateral to the hand of button press during hallucinations, and auditory cortex during acoustic stimulation. Our primary aim was to identify components of auditory cortex activity during hallucinations, and to compare these components to those of sensorimotor cortex activation during hallucinations and auditory cortex activation during acoustic stimulation. COIs were selected using standardized spatial templates based on a probabilistic map (Rademacher et al., 2001) and standardized coordinates (Talairach and Tournoux, 1988) of the bilateral auditory and sensorimotor cortex, respectively. A selected component was labeled as COI when 1) there were no obvious signs of artifactual contributions such as head movements or suprathreshold Z-values in areas known to be prone to large measurement artifacts (e.g., the sinuses), and 2) clusters of 


\section{Chapter 2}

suprathreshold voxels within the template areas were of 6 functional voxels or larger.

The temporal pattern of the hallucination episodes of the COls was analysed using BOLD-image plots (Duann et al., 2002), a visualization technique that is derived from the study of single trial variability of eventrelated potentials (Jung et al., 2001). These plots depict the temporal variance across different trials or episodes. The plots were obtained in the following manner. The spatial COI maps were sign-corrected by multiplying the map values with the sign of the correlation coefficient between the COI timecourse and the reference timecourse. To prevent spurious corrections, the procedure was only executed for correlations higher than $|0.1|$. Then, for each COI the timecourses of the suprathreshold voxels were extracted from the initial data set for further analysis. Note that voxel selection was not restricted to those voxells that fell within the boundaries of the spatial templates, but comprised all suprathreshold voxels within the COI map. Independent component maps can be considered as maps of functional connectivity (Van de Ven et al., 2004), where the voxel values represent the amount of shared information (time-point by time-point) between the voxel timecourses. Therefore, we chose to extract voxel timecourses from the whole map, since this would give a more accurate representation of the temporal information contained within the component. The extracted timecourses were temporally smoothed using a Gaussian kernel of full width at half maximum [FWHM] $=2$ timepoints, and were averaged to obtain the mean COI-activity timecourse. This timecourse was divided by the mean level of activity of the extracted timecourses to obtain units of percent BOLD signal change.

We extracted the BOLD signal from the COI-activity timecourses for each hallucination or acoustic stimulation episode. The time window for an episode was set from one volume prior to the onset of the event up to 24 seconds from the onset. This procedure was the same for all measurements. An episode was discarded if 1) the distance over time between the onset of a hallucination and the end of the previous hallucination was less than 12 seconds, or 2) the maximum percent BOLD signal change within the trial window exceeded $|6|$ percent, in order to avoid the contribution of motion artefacts and large vessels. The trials that survived these criteria were interpolated to units of 1 second using spline interpolation, converted to horizontal color bars with color codes corresponding to the percent signal change values, and were stacked in the plots (Duann et al., 2002). The mean trial timecourse was appended to the plot.

\section{Results}

\section{Independent component analysis}

Table 2.2 presents the descriptive statistics of the hallucination episodes, as reported by the patients through button press. The following results pertain to 
Patient A - Hallucinations

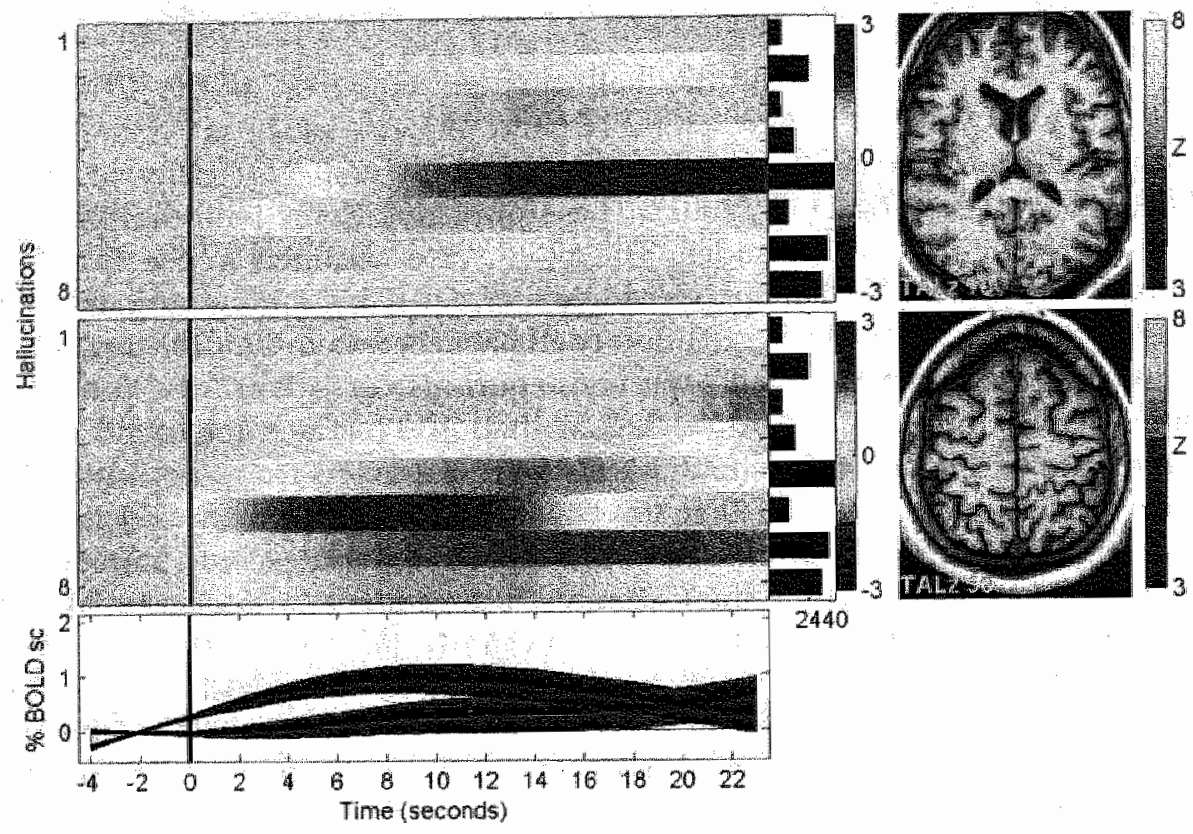

Figure 2.1. BOLD-image plot of the auditory and sensorimotor components of interest during hallucinations of patient $A$. From left to right for each row: 1) the plots with horizontal color bars depict the percent BOLD signal change (\% BOLD sc) for eight hallucination episodes (BOLD-image plot); 2) Attached to the right of each BOLD-image plot is the bar plot for the lengths of each hallucination episode in secs; 3) Attached to the right of the bar plot is the color legend for the BOLD-image plot; 4) On the utmost right the component map is shown superimposed on the anatomical image (left is left the transverse coordinate in standardized space [TALz] in orange). The Z-values of the component map for auditory cortex is shown in red-yellow color scalle and the map values for sensorimotor cortex in dark blue - light blue, each thresholded at $Z=3$. Below the BOLD-image plots the averaged episode timecourses, with 1 standard error of the mean, are shown for the auditory (red) and sensorimotor (blue) components. The plots show that, despite a wide variation in onset, peak and overall shape of the BOLD signals over episodes, on average there is an increase in left auditory cortex. For color version, see page 135.

the first decomposition (see Methods). For patients A, D and E only one COI that encompassed primary and secondary auditory cortex was found (see figures 1,2 and 3). More specifically, for the decomposition of patient A the COI comprised clusters in left Heschl's gyrus, for patient D it comprised bilateral Heschl's gyrus, and for patient E right anterior and lateral Heschl's gyrus and posterior superior temporal gyrus. For patient F, several COIs that contained clusters of high absolute Z-values in auditory cortex were found, of which only one component correlated moderately with the button press response. For 
patients B and C no auditory cortex COI was detected. A COI for sensorimotor cortex contralateral to the hand of button press was found in five out of six patients, with the exception of patient $C$. For each of the acoustic stimulation measurements one COI comprising bilateral auditory cortex was detected (Figure 2.4). Table 2.3 lists the spatial coordinates and cluster sizes of the suprathreshold voxels in the template areas for each COI.

We verified that the selected COIs correctly reflected the BOLD activity related to hallucinations and acoustic stimulation by correlating the COIactivity timecourses with the hallucination or acoustic stimulation reference models. Most correlations were significant $(p<0.05$; see Table 2.4$)$, suggesting that the COIs did indeed reflect the BOLD-responses related to hallucinations or acoustic stimulation.

Table 2.2. Descriptive statistics of the hallucination self-reports of the patients.

\begin{tabular}{ccccccc}
\hline & \multirow{2}{*}{$\begin{array}{c}\text { Hand of } \\
\text { Patient }\end{array}$} & $\begin{array}{c}\text { Nhallucination } \\
\text { button press }\end{array}$ & epochs & & \multicolumn{3}{c}{ Duration of epochs (in volumes) } \\
\cline { 6 - 7 } & Left & 10 & TR & Range & Mean (SD) & $h /$ nvols \\
\hline A & Left & 9 & 4 & $2-10$ & $5.9(3.0)$ & 0.46 \\
B & Left & 23 & 4 & $1-6$ & $3.2(1.6)$ & 0.23 \\
C & Left & 22 & 4 & $1-5$ & $1.5(0.9)$ & 0.28 \\
D & Right & 9 & 2 & $1-23$ & $6.4(5.0)$ & 0.48 \\
E & Right & 54 & 2 & $4-14$ & $6.8(2.9)$ & 0.30 \\
F & & 2 & $1-4$ & $1.9(0.6)$ & 0.42 \\
\hline
\end{tabular}

$\mathrm{TR}=$ time of repetition (in secs); h/nvols = proportion of measured volumes associated with hallucination self-report.

\section{BOLD-image plots}

The BOLD-image plots of the hallucination measurements for patients $A, D$ and $E$ are depicted in figures 1,2 and 3 . In each figure, the bar plots to the right side of the BOLD-image plots show the duration of each button press response. The panel below the BOLD-image plots shows the averages of the episode-rielated BOLD signals, with the area of one standard error of the mean around the average timecourses (red for auditory; blue for sensorimotor). A transverse image of the component map superimposed on the anatomical image is depicted on the right of each BOLD-image plot (red - orange for auditory; blue - green for sensorimotor). The BOLD-image plots of the hallucinations reveal a large variability in the time of onset, peak and shape of the single episode BOLD responses within the auditory COIs. However, the averages of the single episode responses indicate that BOLD activity increased during episodes of hallucinations. Figure 2.5 shows the BOLD-image plots for the acoustic stimulation sessions of the six patients. 


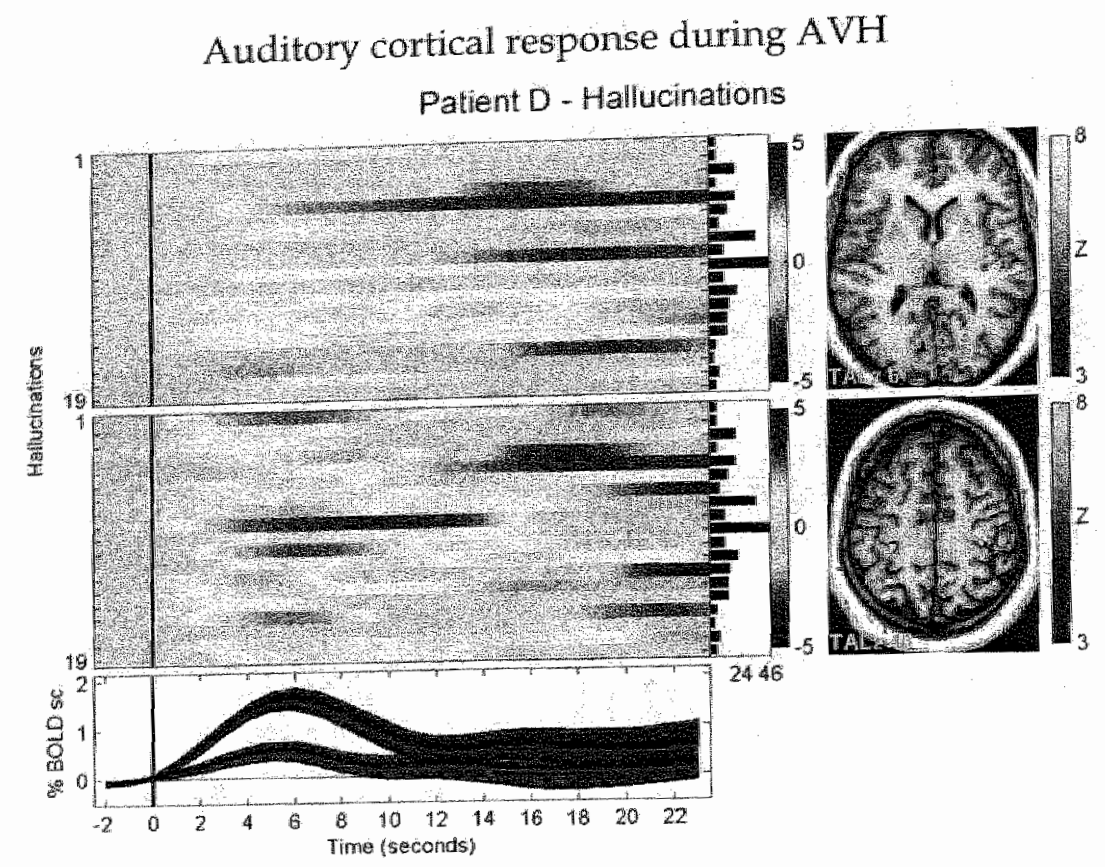

Patient $E$ - Hallucinations

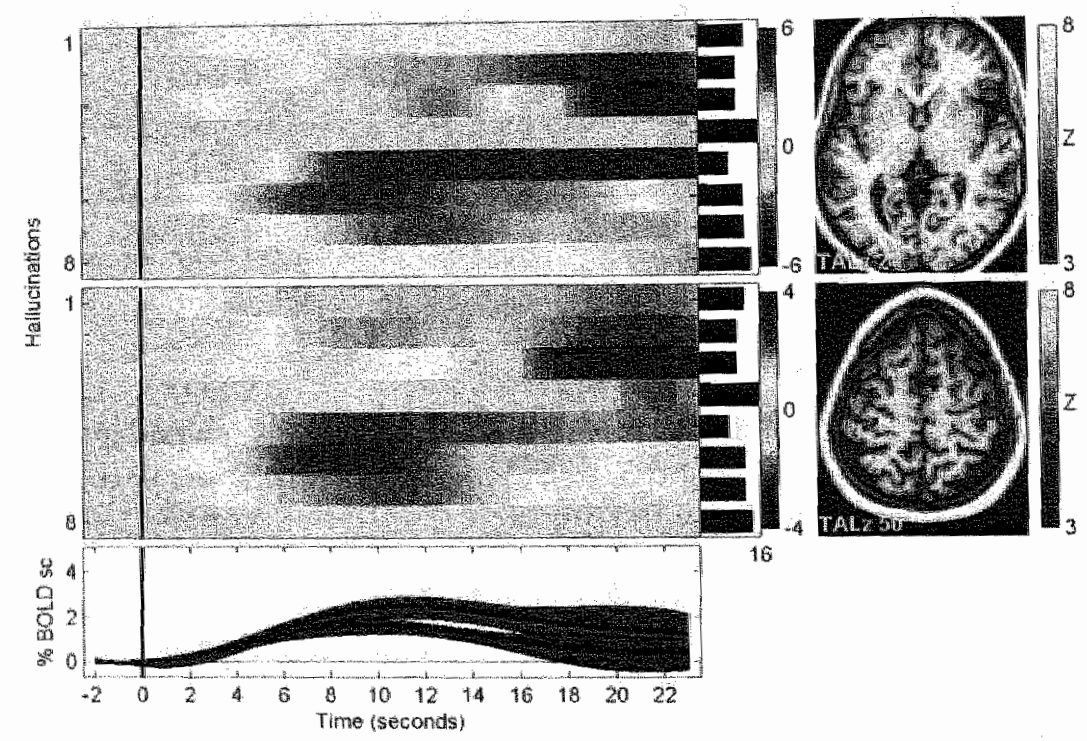

Figure 2.2. BOLD-image plot of the auditory and sensorimotor components of interest during hallucinations of patient $D$. The plots show that for bilateral primary auditory cortex the average BOLD episode response increases in the early phase of the hallucinations. For further detail, see Figure 2.1. For color version, see page 136. 


\section{Chapter 2}

Figure 2.3. BOLD-image plot of the auditory and sensorimotor components of interest during hallucinations of patient $\mathrm{E}$. The plots show that for right primary auditory cortex the average BOLD episode response increases in the early phase of the hallucinations. For further detail see Fugure 2.1. For color version, see page 120 .

Table 2.3. Standardized spatial coordinates $[x, y, z]$ of the peak $Z$-value and cluster size $(\mathrm{mm})$ of the regions of interest in the obtained components of interest during hallucinations and acoustic stimulation.

\begin{tabular}{|c|c|c|c|c|c|c|c|c|c|}
\hline \multirow[b]{2}{*}{ Patient } & \multirow[b]{2}{*}{ Sn } & \multirow{2}{*}{\multicolumn{2}{|c|}{$\begin{array}{c}\text { area } \\
+\end{array}$}} & \multicolumn{3}{|c|}{ Left } & \multicolumn{3}{|c|}{ Right } \\
\hline & & & & $x_{i} y, z$ & $\max _{z}$ & size & $x_{s} y, z$ & $\frac{\max }{Z}$ & size \\
\hline \multirow[t]{6}{*}{ A } & $\mathrm{H}$ & $\mathrm{HG}$ & & $-37,-23,9$ & 7.3 & 621 & $56,-5,0$ & 4.9 & 378 \\
\hline & & & PTP & $-52,-32,12$ & 5.1 & 459 & & & \\
\hline & & SMC & & & & & $44,-23,60$ & 5.7 & 459 \\
\hline & & & & & & & $44,-17,60$ & 7.7 & 270 \\
\hline & 5 & $\mathrm{HG}$ & & $-61,-17,6$ & 11.9 & 540 & & & \\
\hline & & & STG & & & & $65,-35,9$ & 11.3 & 2430 \\
\hline \multirow[t]{3}{*}{ B } & $\mathrm{H}$ & SMC & & & & & $41,-17,42$ & 5.3 & 243 \\
\hline & $S$ & $\mathrm{HG}$ & & $-64,-26,18$ & 20.8 & 2214 & $62,-11,9$ & 10.3 & 2889 \\
\hline & & & & $-61,-2,0$ & 8.9 & 3024 & & & \\
\hline C & $S$ & $\mathrm{HG}$ & & $-64,-20,3$ & 4.2 & 405 & & & \\
\hline \multirow[t]{4}{*}{$\mathrm{D}$} & $\mathrm{H}$ & $\mathrm{HG}$ & & $-46,-23,24$ & 3.6 & 216 & $47,-20,6$ & 5.6 & 378 \\
\hline & & & PTP & $-58,-23,24$ & 8.8 & 2862 & & & \\
\hline & & SMC & & & & & $38,-35,57$ & 14.6 & 5670 \\
\hline & 5 & $\mathrm{HG}$ & & $-52,-23,0$ & 9.5 & 5562 & $53,-32,6$ & 9.1 & 3834 \\
\hline \multirow[t]{5}{*}{ E } & $\mathrm{H}$ & $\begin{array}{l}\mathrm{HG} / \\
\text { STC }\end{array}$ & & & & & $53,4,-6$ & 26.1 & 5076 \\
\hline & & SMC & & $-28,-20,63$ & 7.4 & 621 & & & \\
\hline & & & & $-46,-23,51$ & 4.2 & 189 & & & \\
\hline & $s$ & $\mathrm{HG}$ & & $-46,-29,6$ & 3.4 & 216 & $41,-23,3$ & 4.5 & 513 \\
\hline & & & STG & & & & $53,4,-9$ & 16.4 & 3834 \\
\hline \multirow[t]{4}{*}{$\mathrm{F}$} & $H$ & SMC & & $-31,-32,66$ & 4.9 & 297 & & & \\
\hline & & & & $-37,-32,42$ & 7.5 & 2511 & & & \\
\hline & $\mathrm{s}$ & $\begin{array}{l}\mathrm{HG} / \\
\mathrm{STG}\end{array}$ & & $-61,-8,9$ & 11.4 & 3996 & $59,-2,6$ & 4.9 & 243 \\
\hline & & & & & & & $44,-26,12$ & 7.9 & 1755 \\
\hline
\end{tabular}

Notes: $S n=$ session; $\max Z=$ maximum $Z$-value; $S M C=$ sensorimotor cortex; $H G=$ Heschl's gyrus; $\mathrm{STG}=$ superior temporal gyrus; $\mathrm{PTP}=$ posterior temporal plane; $H=$ hallucinations; $S=$ acoustic stimulation; areat = additional area of suprathreshold Z-values that overlaps with the region of interest. 


\section{Auditory cortical response during AVH}

In a next step, we descriptively examined whether hallucination parameters were related to the detection of an auditory COI during hallucinations. To this end, we plotted the mean duration of hallucinations, the percentage of self reports during the measurement and the number of reported hallucinations for the six patients (Figure 2.5). The average duration of hallucinations seemed to be most strongly related to the detection of an auditory $C O C$. The scores on this parameter was the lowest for patient $C$, for whom no auditory or sensorimotor COI was detected.

\section{Post-hoc comparisons}

When the three decompositions (number of components: a quarter and a third of number of volumes, and 100 regardless of number of volumes) were compared, a few differences emerged. For the additional decompositions the auditory COI during hallucinations of patient A comprised bilateral Heschl's gyrus. Clusters in bilateral Heschl's gyrus were found for the initial decomposition when the $Z$-threshold for visualization was lowered. The additional decompositions of the hallucination measurement of patient $F$ revealed components with suprathreshold clusters in left Heschl's gyrus, but was not significantly correlated with the reference timecourse. Because of these marked differences between the decompositions it was concluded that the hallucination measurement of patient $F$ does not comprise specific auditory cortex activity during hallucinations. All other results of the initial decompositions were replicated in the additional decompositions. Figure 2.6A shows the amount of overlap of the auditory COIs of hallucinations between the different decompositions of patients A, D and E (see Table 2.5 for spatial correlations between COIs of the different decompositions).

Cortex-based correlation analysis of the hallucination datasets using delayed and convolved (Boynton et al, 1996) reference time courses of the button presses showed that activity increased in primary and secondary auditory cortex during hallucinations for patients $A$ and $\mathbb{E}$ (Figure 2.6B), but not for patient $\mathrm{D}$. Correlation maps were converted to a $\mathrm{Z}$-distribution, and the correlation threshold was set in such a way that the number of significantly active voxels equaled the total number of suprathreshold voxels within the auditory and sensorimotor COI. A region of interest analysis of left and right Heschl's gyrus of patient $D$, based on anatomical landmarks, verified that bilateral putative PAC is active during AVH as well as acoustic stimulation (Figure 2.7).

Finally, estimation of event-related changes in BOLD signal for the suprathreshold clusters in left and right Heschl's gyrus of each auditory COI during hallucinations showed that timecourses from these clusters reflected the average COI-activity timecourse (Figure 2.7). 


\section{Chapter 2}
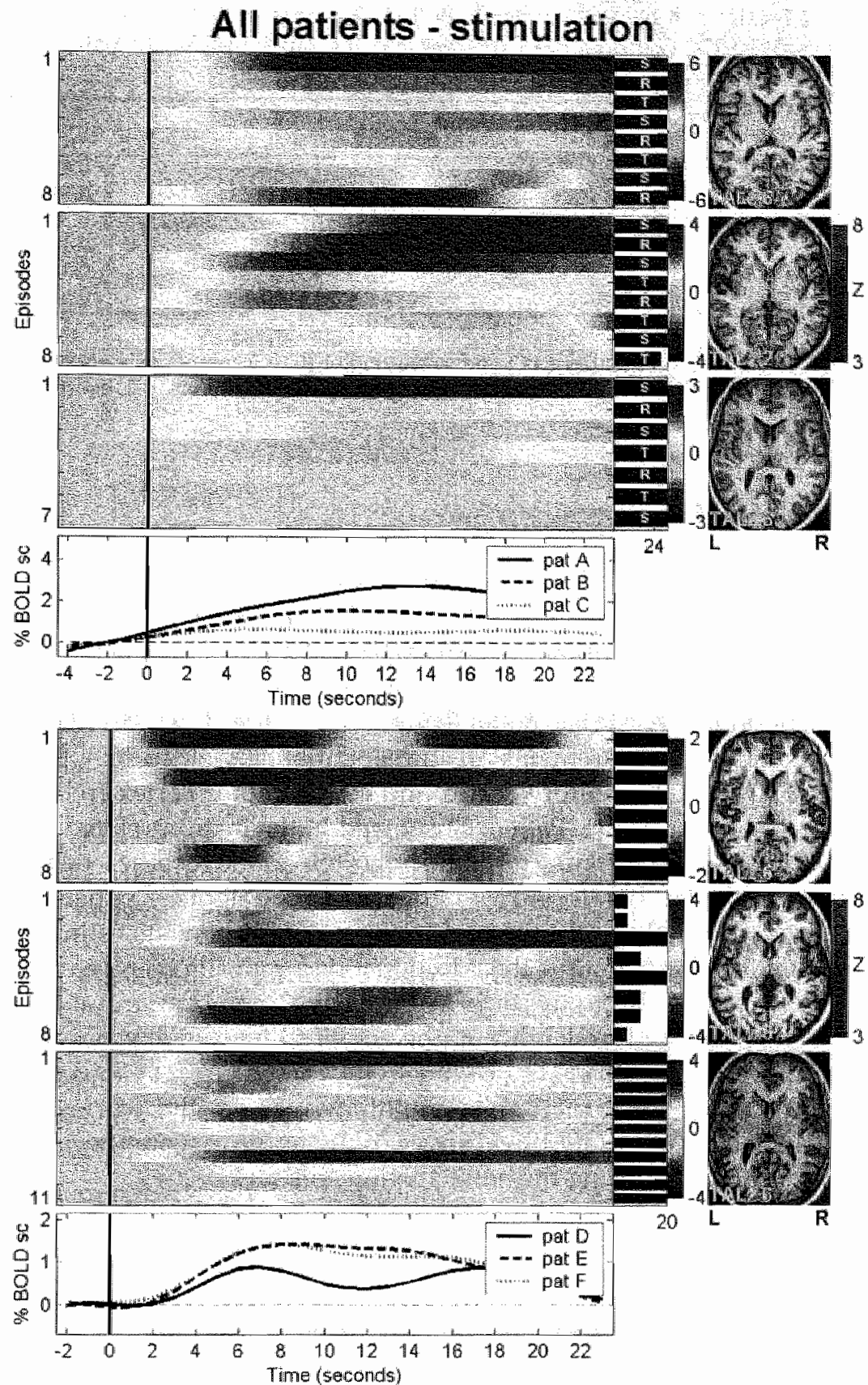

Figure 2.4. BOLD-image plots of the auditory cortex during acoustic stimulation of the six patients. The plots are grouped for study 1 and study 2 (see text). For patients $A, B$ and $C: S=$ speech; $R=$ reversed speech; $T=$ tones. All acoustic stimuli for patients $\mathbb{D}, \mathrm{E}$ and $\mathrm{F}$ comprised tones. For further detail, see figure 2.1. For color version, see page 137. 
Table 2.4. Correlations between average BOLD timecourse obtained from the positive voxels of the component of interest and the button press/acoustic stimulation reference model.

\begin{tabular}{|c|c|c|c|}
\hline \multirow[t]{2}{*}{ Patient } & \multicolumn{2}{|l|}{$\mathrm{AVH}$} & AS \\
\hline & $A C$ & SMC & $\overline{A C}$ \\
\hline A & $0.12^{\mathrm{b}}$ & 0.41 & 0.65 \\
\hline Bi & - & 0.15 & 0.74 \\
\hline 6 & $\therefore$ & - & 0.28 \\
\hline$D$ & 0.20 & 0.31 & 0.46 \\
\hline $\mathrm{E}$ & 0.39 & 0.53 & 0.33 \\
\hline I. & - & 0.11 & 0.55 \\
\hline
\end{tabular}

Components of interest are auditory (AC) and sensorimotor (SMC) components obtained from the hallucination (AVH) and acoustic stimulation (AS) sessions. Notes: all $p$ 's $<0.07$, except " $p<0.05$; to not significant

\section{Discussion}

We analysed data of six hallucinating schizophrenia patients using spatial independent component analysis (sICA), and characterised the activity in the left and right auditory cortex, including Heschl's gyrus, the site of primary auditory cortex (PAC) in humans (Rademacher et al., 2001; Formisano et al., 2003), during auditory verbal hallucinations (AVH) and acoustic stimulation. The hallucination measurements of three patients yielded components of interest (COIs) in both auditory cortex and sensorimotor cortex (related to the self-report by button press). Patient D showed hallucination-related activity in bilateral primary and secondary auditory cortex and two other patients only unilaterally (in the left hemispere for patient $A$, in the right for patient $\mathbb{E}$ ). These findings proved to hold with different number of components estimated, with the exception that for patient $A$ the additional decompositions revealed clusters in bilateral PAC. COI-activity timecourses were extracted from the spatial maps, and the temporal variability of the activity timecourse was investigated using BOLD-image plots. The COL-activity timecourses showed a large variability in time of onset and amplitude across the various epochs. Nevertheless, all COI-activity timecourses of the auditory components showed an increase in activity during the hallucination episodes.

Table 2.5. Spatial correlations ( $r$ ) between initial decomposition (1) of $1 / 4 \times$ number of volumes and additional decompositions ( 2 and 3 ) of $1 / 3 \times$ number of volumes and 100 components regardless of number of volumes.

\begin{tabular}{c|ccc}
\hline & $\mathrm{A}$ & $\mathrm{D}$ & $\mathrm{E}$ \\
\hline $\mathrm{r}_{1,2}$ & 0.53 & 0.66 & 0.79 \\
$\mathrm{r}_{13}$ & $0.32^{*}$ & 0.73 & 0.75 \\
\hline
\end{tabular}

Notes: * component of $3^{\text {nt }}$ decomposition comprises bilateral Heschl's gyrus at threshold of $Z=3$. 


\section{Chapter 2}
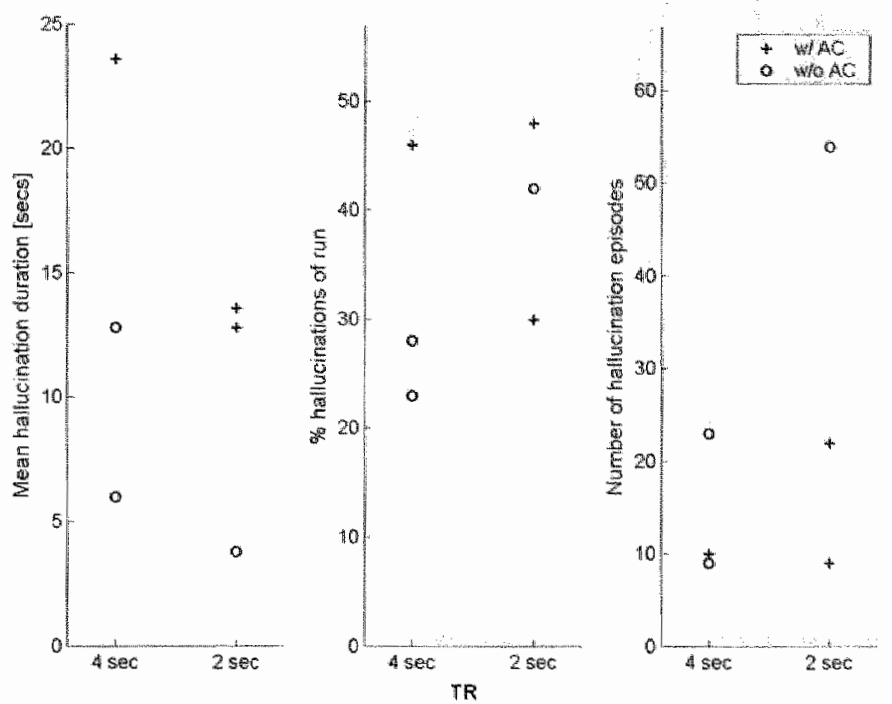

Figure 2.5. Scatter plots. From left to right: scatter plots for mean hallucination duration (in secs), percentage of scanning time during which hallucinations were reported, and total number of hallucination episodes for the six patients. TR = repetition time; $w / \circ \mathrm{AC}=$ without detection of an auditory component.

Activity in bilateral auditory cortex was found for all acoustic stimulation measurements. Although the auditory COIs of both the hallucination and acoustic stimulation included Heschl's gyrus, the COls differed between the sessions in the size of the suprathreshold clusters and the presence of clusters outside of the auditory cortex. Taken together, these results indicate PAC activity during AVH (Bentaleb et al., 2002; Dierks et al., 1999; Lennox et al., 2000) in a number of patients, but do not indicate a specific involvement of the dominant hemisphere (Dierks et al., 1999). Furthermore, the results show that data-driven analysis may complement hypothesis-driven analyses for complex and unpredictable phenomena such as hallucinations.

The hemispheric side of sensorimotor cortex activity due to the button presses did not seem to be related to the hemispheric side of the auditory cortex activity. This suggests that the auditory signal is not in some way influenced by sensorimotor activity, and is supported by the finding that the auditory and sensorimotor cortex activity were contained in two separate components. For patient $\mathrm{C}$ we failed to detect a sensorimotor $\mathrm{COI}$ related to the button presses. Post-hoc correlation analysis revealed small clusters of activity in sensorimotor cortex only at a low threshold ( $\mathrm{p}<0.0017$, uncorrected), which may indicate that the overall functional contrast -to-noise in this subject was too low for activity associated with hallucinations to be detected. 

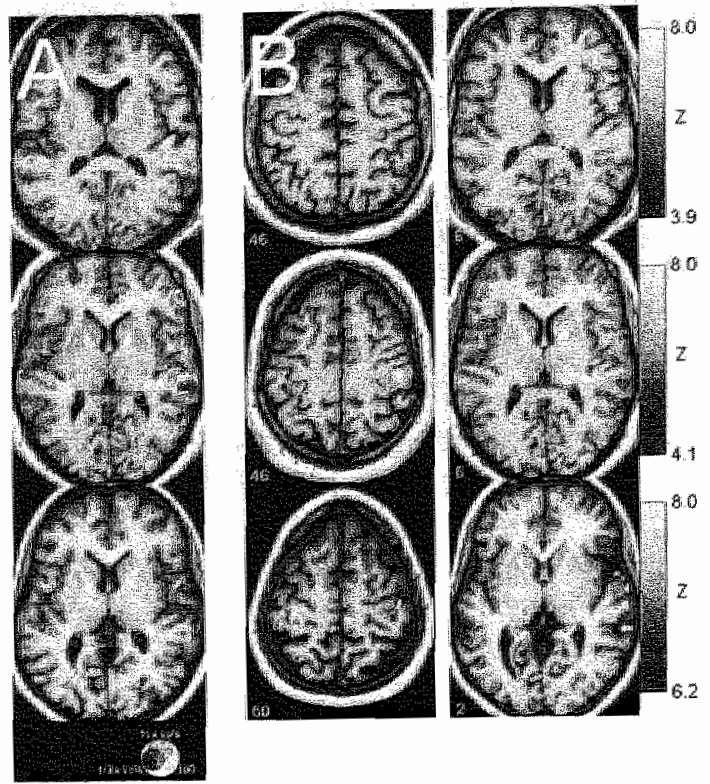

Figure 2.6. A: Degree of spatial overlap between auditory components of interest during hallucinations of patients $A, D$ and $E$. Maps were thresholded at $Z=3$. Initial decomposition $=1 / 4 \times$ number of volumes (blue); additional decompositions = $1 / 3 \times$ number of volumes (red) and 100 independent components regardless of number of volumes (yellow). Orange and purple indicate overlap across decompositions. Left hemisphere is depicted on the left side. For patient $A$, bilateral Heschl's gyrus was found for the decomposition of 100 components. $B$ : Correlation maps of auditory hallucinations of patients $A, D$ and E. Maps are thresholded at the number of correlating voxels $=$ number of suprathreshold component of interest voxels. Only patients $A$ and $\mathbf{E}$ show activity during hallucinations in Heschl's gyrus. For color version, see page 138.

Dierks at al. (1999) suggested that the activity in PAC represents the high degree of vividness of the hallucination that is commonly reported by the patiants. This interpretation would be supported by a lack of activity in PAC during the generation and manipulation of an auditory verbal (Shergill et al., 2001) or musical (Halpern and Zatorre, 1999) mental image in healthy individuals because hallucinations are typically experienced as more vivid and real than mental images. The results of Engelien et al. (2000), who showed that a neurologic patient with lesions that comprised bilateral Heschl's gyrus could report the coarse characteristics of acoustic stimuli (e.g., on- and offset of a stimulus), but not their detailed features, conform to this view. Vivid recall of memorized sounds activated secondary, but not primary auditory cortex in healthy subjects (Wheeler et al., 2000), which may suggest that PAC activity during hallucinations is of a pathological nature. It is currently not known, however, whether similar increased activity in primary sensory cortex during imagery or memory recall in healthy subjects would also induce a 


\section{Chapter 2}

misidentification of the source (internal vs. external) of the imagined or recalled item. However, the activity in auditory cortex during hallucinations may also reflect attentional responses to the presence of a hallucination because activity in PAC can be enhanced through attentional modulation (Jäncke et al. 1999). The saliency of an $A V H$, as well as the task instruction of monitoring the hallucination events, may thus have resulted in the temporary enhancement of cortical activity.

Our study shows that the on- and offset of auditory cortex activity might deviate considerably from the button press report. Sources of this variability may be found in patient compliance, task instruction and complexity of auditory cortex activity. Firstly, the patients' self-reports themselves may have been inaccurate. The inherent difficulty to distinguish a hallucination from a real experience is a prominent characteristic of hallucinations as a clinical phenomenon (American Psychiatric Association, 1994), and hallucinations have been associated with a failure to monitor and identify the source of internally generated images (Blakemore et al., 2000). Although the patients reported that they could reliably detect their hallucinations, we could not verify their reported accuracy, or whether there was a bias towards reporting a hallucination in case of doubt. In addition, the activity of PAC may not be a universal characteristic of hallucination-related brain activity, reflecting the phenomenological variability of AVHs (Stephane et al., 2003). For example, highly detailed and vivid verbal hallucinations may recruit processing capacity of PAC to a larger extent than hallucinations where the voices are distant and the linguistic content is vague.

Secondly, the variability of the neural signal may be related to the explicit instruction to monitor and respond to the hallucination events. Attentional effects within auditory cortex (Jäncke et al., 1999) that are secondary to the hallucination may have altered the BOLD response with respect to the button presses.

Thirdly, the auditory cortical response to stimuli of varying duration has proven to be complex in healthy subjects (Harms \& Melcher, 2003; Seifritz et al., 2002) as well as in the patients in this study. This complexity may increase further in the case of auditory percepts of a pathophysiological nature. The primary and secondary auditory cortex show anatomical (Hirayasu et al.., 2002) and functional (Calhoun et al., 2004) abnormalities in schizophrenia patients, in comparison to healthy subjects, which may add to the complexity of the neural response of AVH.

Many of these difficulties are currently inherent to the study of hallucinations in general. In all of these cases a sequence of self-reports may prove to be an inaccurate model for hallucination-related brain activity. Because sICA does not depend on any predefined model of brain activity, it can complement conventional (hypothesis-driven) approaches in the attempt to identify the neural signal that is associated with AVH. 

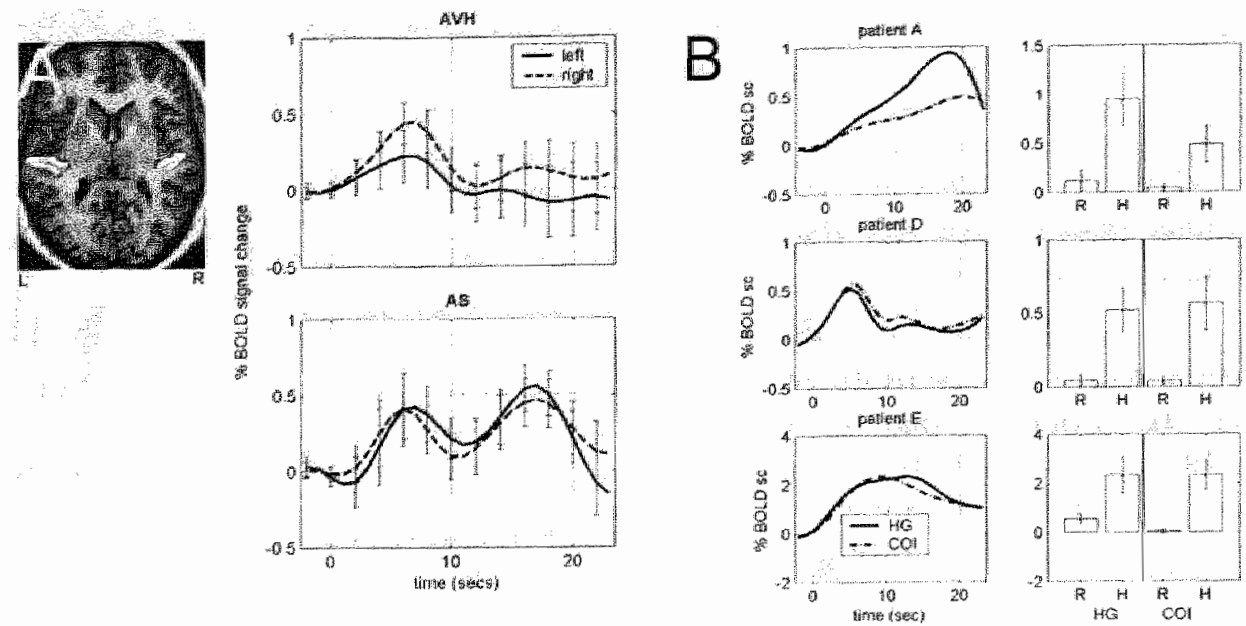

Figure 2.7. A: Event-related averages of activity in left and right Heschl's gyrus (delineated in white in the anatomical image) during hallucinations (AVH) and acoustic stimulation (AS) of patient D. Average timecourses have similar shapes to the BOLD-image averages for AVH (Figure 2.2) and AS (Figure 2.4). B: Event-related plots (left) of the COl-activity timecourse of the whole component (COI) and of clusters in left and right Heschl's gyrus (HO) of patients A, D and E. Bar charts (right) show mean and standard error of the peak amplitude. $H G=$ Heschl $^{s}$ s gyrus; $C O I=$ component of interest; $\mathbb{R}=$ no hallucination; $\mathrm{H}=$ during hallucinations.

The detection of an auditory cortex COI during hallucinations seemed related to the average length of episodes of hallucinations. This finding would suggest that auditory cortex becomes active, or rather that its activity only becomes detectable with standard CMRI, if episodes of hallucinations last

long enough. In line with previous suggestions (Dierks et al , 1999), the average length of hallucination episodes should be at least 10 seconds in order to be reliably detected. A higher sampling rate of the measurements, i.e., a lower TR, in combination with higher magnetic field strengths may be important in order to catch more transient episodes. Furthermore, higher sampling rates allow for the analysis of temporal relations between cortical areas. Further studies are needed to investigate whether faster measurements, perhaps of only the auditory cortex, could improve detection of brain activity during hallucinations.

The current study is limited by several factors. First of all, the sample size was rather small, which is a general problem of fMRI studies with hallucinating psychiatric patients because only few of them are able or willing to report on their hallucinations while in the tomograph. Perhaps the use of data-driven analysis methods may help to alleviate the current necessity for patients to overtly respond to their hallucinations, which may facilitate patient recruitment in the future. The relatively small number of patients, difference in design 
parameters across patients and interindividual variability make the generalization of our findings to the schizophrenia population as a whole impossible. Furthermore, we did not use specialized methods to exactly. identify and analyse tonotopic primary auditory cortical fields (Formisano et al., 2003), and therefore cannot exclude that our findings partly reflect physiological processes unrelated to PAC. Finally, our results may be influenced by limitations of sICA. It is not known if the assumption of ICA, which states that the observed data is a linear combination of sources, is completely accurate for fMRI data, and how the decomposition is affected if this assumption does not hold. Also, the choice of the number of components for a decomposition is of particular importance. Under-estimating the number of sources in the data may result in an erroneous decomposition of the data, while over-estimation may result in artifactual separation of one source across multiple components (Formisano et al., 2004). We decomposed the individual datasets using three different choices for number of components, and found that the COI maps remained largely stable across decompositions. A region of interest analysis of bilateral Heschl's gyrus of patient $D$ confirmed the corresponding COI of bilateral auditory cortex activity during hallucinations. Therefore, it is not likely that our results are compromised by a faulty decomposition of the data structure. It should be noted, however, that any interpretation of sICA maps has to be given with caution because the method, without any further assumptions about the nature of the signals and the noice, does not provide any measure of probability of error, nor does it assess the functional significance of the signal of interest. However, this potential drawback has the benefit of revealing unpredicted patterns in the data, on which basis new hypotheses can be generated.

In conclusion, by applying sICA to FMRI data we showed brain activity in the auditory cortex, including $\mathrm{PAC}$, of both hemispheres during $\mathrm{AVH}$ in a subsample of patients. The activity patterns reveal a large temporal variability, which makes the development of an appropriate pre-defined model of brain activity very complicated. Whether auditory cortex activity can be detected during hallucinations, may be related to the length of the hallucination episodes. SICA has the potential to identify these activity patterns without the necessity of any model of activity, which makes it a very powerful tool for the analysis of neurophysiological signals that are related to the subjective experience of psychiatric patients. 


\section{Acknowledgements}

Prof K. Maurer, head of the Dept. of Psychiatry of Frankffurt University is gratefully acknowledged for constant support. Prof. F. Zanella and Dr. H. Lanfermann of the Dept. of Neuroradiology of Frankfurt University kindly provided access to the MR tomograph. The authors would like to extend their particular thanks to the patients who participated in the study. 
Chapter 3

Exploring connectivity of auditory verbal hallucinations 


\section{Abstract}

A number of neuroimaging studies of auditory verbal hallucinations (AVH) have showed activity during hallucinations in cortical areas associated with language and auditory-sensory processes. In some cases, primary auditory cortex was found active during AVH. These findings have been interpreted as indicating a widespread network of cortical areas that subserves the experience of AVH. However, so far no analysis on the network-level of brain activity during AVH has been conducted. In this study we aimed to analyse brain activity during AVH in terms of functional connectivity, as assessed by spatial independent component analysis, or SICA. SICA decomposes a functional dataset into spatially independent components, which can be interpreted as functional connectivity components. The functional data of five schizophrenia patients who experienced episodes of AVH during functional measurements were decomposed, and spatial components that contained areas of interest inferior frontal gyrus, posterior temporal plane, Heschl's gyrus and middle temporal gyrus - were analysed. Four of five patients revealed spatial connectivity components of left, right or bilateral Heschl's gyrus, the putative cortical site of primary auditory cortex. All patients revealed a connectivity component comprising left lateralized inferior frontal gyrus and posterior temporal plane, as well as inferior parietal and medial frontal areas. The timecourses related to these components showed peak activity during AVH. In addition, a separate component was found for four of five patients that comprised right middle temporal gyrus. In contrast to other single-case reports, no systematic difference in onset of activity was found between these components. In conclusion, several spatiotemporally distinct cortical networks contributed to the experience of AVH, while these networks did not differ from one another in terms of time of onset of activity. 


\section{Introduction}

Auditory verbal hallucinations (AVH) are a core symptom in schizophrenia (American Psychiatric Association, 1994), with a prevalence of over $65 \%$ (Frith, 1999). Functional magnetic resonance imaging (fMRI) and positron emission tomography (PET) studies have associated AVH with increased activity in subcortical and limbic structures (Silbersweig et al., 1995), and language processing and monitoring areas (Shergill et al., 2001), including superior temporal gyrus and inferior frontal gyrus. In addition, a few studies provided evidence of the involvement of primary auditory cortex of the dominant hemisphere in AVH (Dierks et al., 1999; see chapter 2 of this thesis). These functional imaging findings, together with anatomical results (e.g., Hubl et al., 2004; Levitan et al., 1999) suggest specific impairments related to hallucinations in the left hemisphere, which is in concordance with hypothesized cognitive impairments in language processing and monitoring (Cahill \& Frith, 1996; Stephane et al., 2001). However, a small number of studies indicated specific impairments of the early auditory processing system in the right hemisphere (McKay et al., 2000; Wible et al., 2001).

Recently, a small number of case studies suggested that activity in areas that are activated during hallucinations is temporally structured, where right middle temporal gyrus may be active several seconds before a report of hallucinations is made (Shergill et al., 2005; Lennox et al., 1999).

Up to date, functional neuroimaging studies that investigated the functional anatomy of auditory hallucinations used activity-related analysis (Bandettini et al., 1993; Friston, 1996), where localized brain areas that show activity simillar to a predefined model of brain activity are identified. Here, the predefined temporal profile, which constitutes the design matrix, represents a combination of prior knowledge of stimulus presentation and parameters that estimate hemodynamic response properties, such as smoothness and delay of the response (Boynton et al., 1996). In contrast, the connectivity-related aspects of hallucinations measured on-line have not been studied. Networks of areas functionally associated with hallucinations have so far been assessed as the conglomerate of regionally activated (i.e., conforming to a predefined model) areas. This approach does not quantify a degree of shared information between brain areas, which may provide additional insights, such as the presence of a set of networks with different temporal characteristics. In this context the concept of functional connectivity is exploited, which is formally defined as the timepoint-by-timepoint correlation between spatially segragated brain areas (Friston, 1996). Networks are here defined as the cluster of strongly covarying (i.e., highly synchronized) areas. The characteristics of these networks can then be further investigated. 
Functional connectivity can be assessed by using spatial independent component analysis (sICA: McKeown et al., 1998b). The assumption of independence is much stronger than uncorrelatedness (McKeown et al., 1998b) and results in the estimation of components with non-Gaussian probability distributions. A component that consists of multiple, spatially segragated areas can be considered as a functionally connected network (Van de Ven et al., 2004, Yang \& Rapajakse, 2004). The data-driven nature of sICA can be exploited to probe for previously unmodelled spatiotemporal networks that may be related to the experience of AVH. In addition, sICA can be used to probe spatiotemporal brain responses to randomly occurring changes in subjective experiences. Castelo-Branco et al. (2002) found activity in the human visual motion complex (area MT/V5) during subjectively experienced changes in direction of motion, which is not easily modelled using hypothesis-driven analysis.

In this study, we attempted to reveal networks of connectivity that may underlie auditory verball hallucinations, and the temporal relations between these networks. For the analysis of connectivity we used sICA. We focused on networks of perceptual and language-related areas, which included left and right Heschl's gyrus, left inferior frontal gyrus and the posterior temporal plane of the left superior temporal gyrus.

\section{Materials and methods}

\section{Subjects}

Five in- and outpatients diagnosed with paranoid schizophrenia participated in the study (mean [S.D.] age $=31.8$ [5.5] years; one female; four patients were right handed). Four of the five patients experienced AVH that proved resistant against antipsychotic medication. One patient heard voices that commanded him to engage in all kinds of physical activities (e.g., running down the stairs). Another patient experienced insulting and derogatory comments. The other three patients experienced a variety of comments and statements. In all cases, the voices were neyond the patient's control. Four of the patients were taking atypical antipsychotic medication. One patient had temporarilly stopped medication therapy, with the approval of the treating psychiatrist. All patients gave written informed consent before any measurement was conducted.

\section{Imaging parameters}

Patients were required to report on their auditory hallucinations by pressing a button whilst in the scanner. Two patients pressed with the left hand, three patients pressed with the right hand. No visual or auditory stimulus was presented during these sessions. 


\section{Chapter 3}
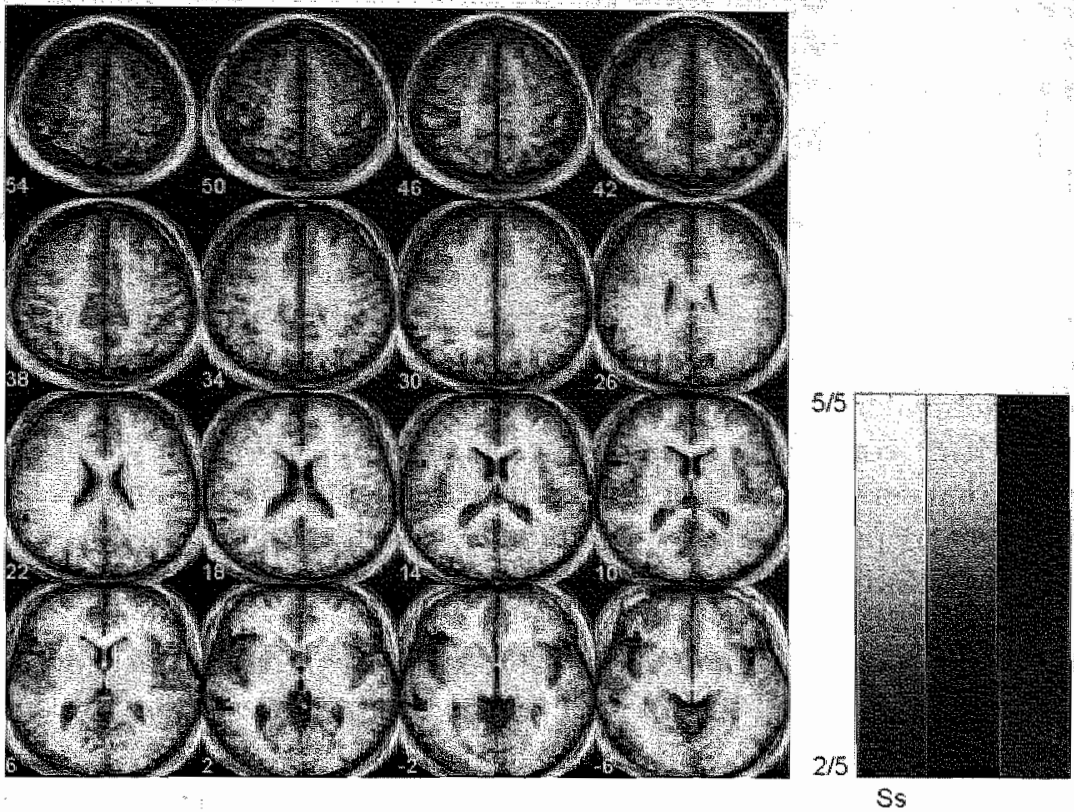

Figure 3.1. Transverse slices of the component maps. Auditory (red-yellow), sensorimotor (dark blue - light blue) and fronto-temporal (pink - blue) component maps are superimposed upon the averaged anatomical image. Voxel values (Ss) indicate the proportion of patients $(\mathrm{n}=5)$ with suprathreshold voxels $(Z \geq 2.6$ ). Left hemisphere is depicted on the left side in the slices. For color version, see page 139 .

Functional and anatomical measurements were conducted using a $1.5 \mathrm{~T}$ Siemens Vision Tomograph (Erlangen, Germany), using an echo-planarimaging [EPI] sequence (time of repetition/echo time [TR/TE] $=2000 / 60 \mathrm{~ms} ; 16$ axial slices; gap thickness $=5 \mathrm{~mm}$; matrix size $\left.=64 \times 64 \mathrm{~mm}^{2}\right)$. The field of view [FoV] ranged from $200 \times 200 \mathrm{~mm}^{2}$ to $240 \times 240 \mathrm{~mm}^{2}$, depending on the head size. For each patient an anatomical three-dimensional (3-D) image was obtained using a magnetization-prepared rapid-acquisition-gradient-echo (MPRAGE) sequence (voxel size $=1 \times 1 \times 1 \mathrm{~mm}^{3}$; matrix size $=256 \times 256 \mathrm{~mm}^{2}$ ).

\section{Data analysis}

The anatomical and functional data were registered, preprocessed and spatially standardized using BrainVoyager 4.8 (Brainlnnovation, Maastricht, The Netherlands). Preprocessing steps for the functional data included slice time correction, standardization into a 3-D space (Talairach \& Tournoux, 1988) with a resampled voxel size of $3 \times 3 \times 3 \mathrm{~mm} 3$, spatial filtering (FWHM $=6 \mathrm{~mm}$ ), and temporal linear trend removal and high-pass filtering (cut-off frequency $=0.007$ $\mathrm{Hz}$ ). The first four volumes were discarded from preprocessing and analysis, 


\section{Functional connectivity of AVH}

resulting in 206 volumes of 16 slices for each dataset. The anatomical images were averaged, and a cortical mask common to all patients was generated, which retained 41,152 voxels for further analysis.

For each patient the subvolume of retained voxels was decomposed using cortex-based spatial ICA (Formisano et al, 2004) into 65 spatial components. Dimension reduction was achieved by using principal component analysis. Spatial templates were created for target areas for each hemisphere, which included auditory cortex (including Heschl's gyrus), sensorimotor cortex and fronto-temporal areas (inferior frontal gyrus and posterior temporal plane, in a single template), based on anatomical landmarks in standardized space (Talairach \& Tournoux, 1988). For each decomposition one component was selected that corresponded best to each spatial template of target areas, and the component maps were then thresholded for visualization at $|Z| \geq 2.6$. Group components were obtained as proportions of overlap of suprathreshold voxels across patients, and were thresholded for visualization at an overlap of two out of five patients. Component activity timecourses were obtained by extracting and averaging the timecourses of positive suprathreshold voxels for each component:

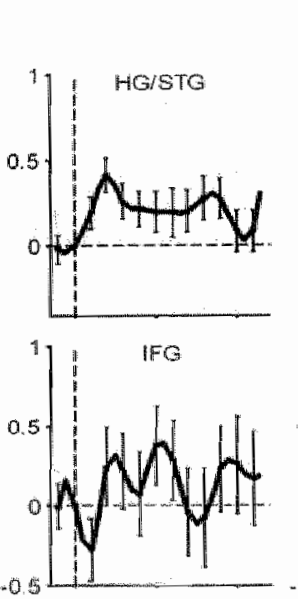

Left
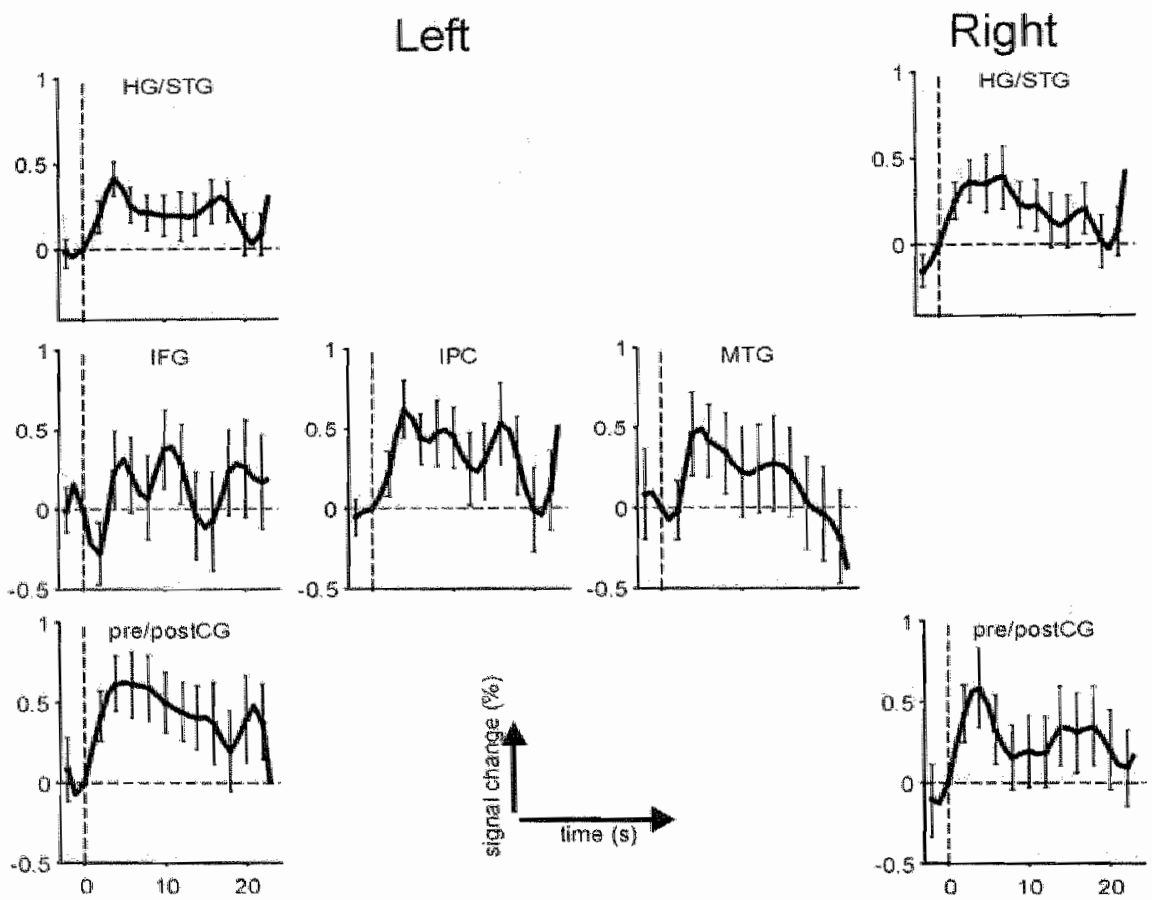

Figure 3.2. Event-related averages and standard error of the mean (S.E.M.) of target areas. Areas are generated by thresholding the amount of overlap at a minimum of 3 patients. For visualization the averages are interpolated to a temporal resolution of $\mathbb{1} \mathrm{s}$. For abbreviations, see Table 3.1 . 


\section{Chapter 3}

Event-related averages for target cortical areas in the component maps were obtained by 1) extracting and averaging voxel timecourses of voxels with a substantial amount of overlap across individual maps (overlap of 3 out of 5 patients), and 2) averaging across hallucination epochs where the minimum allowed distance between the start of an event and the end of the preceeding event was 8 seconds, and maximum percent signal change was 8 percent, in order to avoid large vessel contributions.

To further characterize the spatial pattern of the components of interest the hemispheric laterality index (LD) was calculated as

$$
\mathbf{L I}=(\mathbf{L}-\mathbf{R}) /(\mathbf{L}+\mathbf{R})
$$

where $L$ and $\mathbb{R}$ indicate the amount of suprathreshold voxels in the left $(\mathrm{L})$ and right $(R)$ hemisphere.

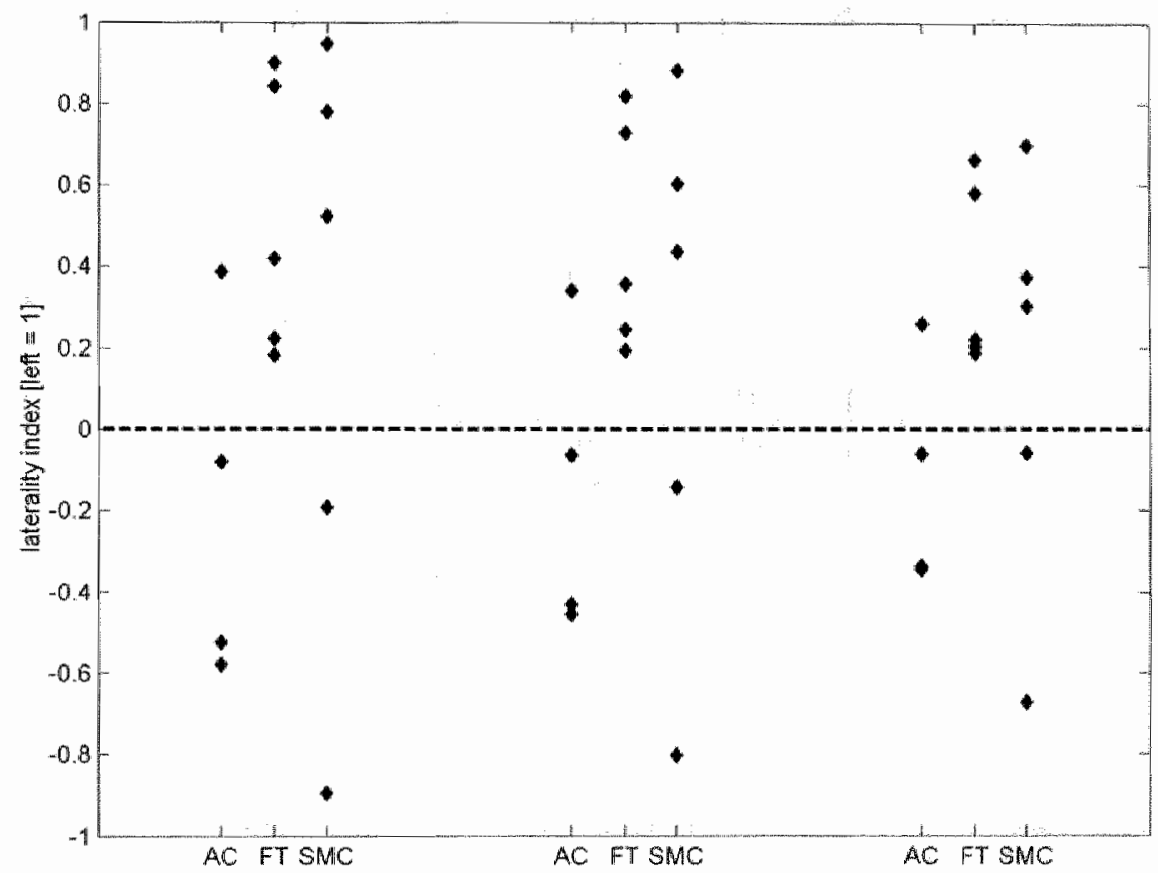

Figure 3.3. Laterality index of the individual spatial maps, at three different thresholds. Laterality index $=(L-R) /(L+R)$, where $L$ and $R$ are the sum of suprathreshold voxels in the left, respectively the right hemisphere, and where the value of 1 indicates complete left-hemisphere lateralization. Left column: spatial threshold of $Z=3$; middle column: $Z=2.6$; right column: $Z=2$. SMC laterality corresponds to the contralateral hand of button press. Despite slightly decreased absolute laterality indices the general pattern of results does not change with changing thresholds. 
Table 3.1. Coordinates $(x, y, z)$ of maximum overlap $(S s)$ and corresponding maximum average $Z$-value of suprathreshold clusters of voxels $(3 \times 3 \times 3 \mathrm{~mm} 3)$ of auditory (AC) sensorimotor (SMC) and fronto-temporal (FT) components of interest (COI) $\mathrm{HG}=\mathrm{Heschl}^{\prime}$ gyrus (a)STG = (anterior) superior temporal gyrus; MTG $=$ middle temporal gyrus, $5 \mathrm{TS}=$ superior temporal sulcus; $\mathrm{INS}=$ insula; $\mathrm{CG}=$ central gyrus; $\mathbb{P G}=$ inferior frontal cortex; MFG = middle frontal gyrus; medFG = medial frontal gyrus, IPL = intra-parietal lobule; (pre)SMA = presupplementary motor area.

\begin{tabular}{|c|c|c|c|c|c|c|c|c|}
\hline \multirow[b]{2}{*}{$\mathrm{COI}$} & \multicolumn{3}{|c|}{ ROI } & \multicolumn{3}{|c|}{ Talairach coordinates } & \multirow[b]{2}{*}{ Ss: } & \multirow[b]{2}{*}{$Z$} \\
\hline & Area & L/R & size. & $x$ & $y$ & $z$ & & \\
\hline \multicolumn{9}{|l|}{$A C$} \\
\hline & aSTG & $\mathrm{R}$ & 31 & 53 & 7 & 6 & 2 & 4.7 \\
\hline & HG/STG & $\mathrm{R}$ & 253 & 59 & -20 & 15 & 3 & 4.6 \\
\hline & HG/PTP & $\mathbb{L}$ & 195 & -40 & -26 & 9 & 3 & 3.3 \\
\hline \multicolumn{9}{|l|}{$\mathrm{FT}$} \\
\hline & aSTG & $R$ & 7 & 47 & 10 & -6 & 2 & 1.5 \\
\hline & IPS & $\mathrm{R}$ & 7 & 44 & -53 & 45 & 2 & 0.7 \\
\hline & $I F G$ & $\mathrm{R}$ & 7 & 47 & 13 & 6 & 2 & 20 \\
\hline & SFG & $\mathrm{R}$ & 30 & 5 & 28 & 57 & 3 & 2.1 \\
\hline & $\begin{array}{l}\text { medFG } \\
\text { medFG/ }\end{array}$ & $\mathrm{R}$ & 23 & 2 & 49 & 21 & 2 & 1.9 \\
\hline & preSMA & $L$ & 35 & -1 & 37 & 39 & 3 & 1.6 \\
\hline & MFG & $\mathrm{L}$ & 6 & -40 & 16 & 45 & 2 & 1.4 \\
\hline & preCG & $\mathrm{L}$ & 20 & -40 & -5 & 48 & 2 & 2.2 \\
\hline & SFG & $L$ & 10 & -40 & 55 & 6 & 2 & 1.1 \\
\hline & SPC & $L$ & 18 & -46 & -47 & 51 & 2 & 3.3 \\
\hline & IFG & $L$ & 165 & -46 & 19 & -6 & 4 & 3.7 \\
\hline & Occ-Cortex & L & 16 & -55 & -65 & 9 & 2 & 1.6 \\
\hline & $\mathbb{A P C}$ & L & 57 & -58 & -47 & 24 & 3 & 2.9 \\
\hline & MTG & $L$ & 71 & -61 & -38 & 0 & 3 & 2.7 \\
\hline & pre/postcG & $\mathbf{R}$ & 281 & 35 & -38 & 48 & 3 & 3.0 \\
\hline & SMA & $\mathrm{R}$ & 17 & 2 & -11 & 54 & 4 & 2.3 \\
\hline & pre/postcG & $\mathrm{L}$ & 614 & -52 & -29 & 45 & 5 & 4.2 \\
\hline
\end{tabular}

\section{Results}

All patients reported that their pattern of hallucinations did not change while lying inside the MR tomograph. The median length of hallucination epochs was 3 volumes (range: $1-19$ volumes; number of events $=90$; mean [S.D.] $=4.5$ [3.8]). In total the patients reported experiencing AVH during $39.5 \%$ of the
measured volumes. 


\section{Chapter 3}

One fronto-temporal (FT) and one sensorimotor (SMC) component (contralateral to the hand of button press) was detected for all patients. One component comprising bilateral auditory cortex component (AC) was detected for three of the five patients. Two ACs were detected for one patient and were averaged to create a single auditory component (temporal correlation $=0.41$ ). For one patient no $\mathrm{AC}$ was detected. Figure 3.1 shows the amount of spatial overlap for each component of interest across the five patients, thresholded at a minimum amount of voxel overlap of two patients ( $S s=2 / 5$ ).

Event-related averages showed increased activity for target cortical areas in these components during hallucination epochs (Figure 3.2; see Table 3.1 for spatial coordinates).

Table 3.2 lists the correllations between the hemodynamically convolved $($ tau $=1.25$, delta $=2.5$ [Boynton et al., 1996]) sequences of button presses and the component of interest-activity timecourses. Only one auditory component and three fronto-temporal components correlated significantly with the button presses.

Computing the LI for each individual map revealed that the four auditory components contained clusters in both hemispheres, but showed a slightly stronger lateralization towards the right hemisphere, while the fronto-temporal components were all strongly left-lateralized (see Figure 3.3 , middle column). Lateralization did not seem to be related to the hand of button press. The pattern of lateralization did not change with different map thresholds (Figure 3.3, left $[Z=3]$ and right columns $[Z=2])$.

Some studies reported activity in cortical areas that preceeded the onset of button presses (Shergill et al., 2005; Lennox et al., 1999). To explore whether a similar pattern was present in our data, we performed a lagged-correlation analysis in steps of $0.1 \mathrm{~s}$, between -10 to 10 seconds around the onset of the button presses (timecourses were interpolated to a resolution of 0.1 second using spline interpolation), using the hemodynamically convolved (Boynton et al., 1996) sequence of button presses. The step associated with the highest correlation varied the most for the $\mathrm{AC}$ and $\mathrm{FT}$ components (see Figure 3.4). However, this variability did not seem systematic. The average lag of the highest correlations for $\mathrm{AC}$ was $-2.3 \mathrm{~s}$, for SMC $-2.42 \mathrm{~s}$, and for FT $-3.46 \mathrm{~s}$. When using the $\mathrm{SMC}$-activity timecourse as reference the highest correlation for $\mathrm{AC}$ was found at an average lag from button press onset of $-0.18 \mathrm{~s}$, and for FT at $0.24 \mathrm{~s}$ (see Figure 3.4). When the AC and FT timecourses were correlated the average lag of the highest correlations was $-0.03 \mathrm{~s}$. 

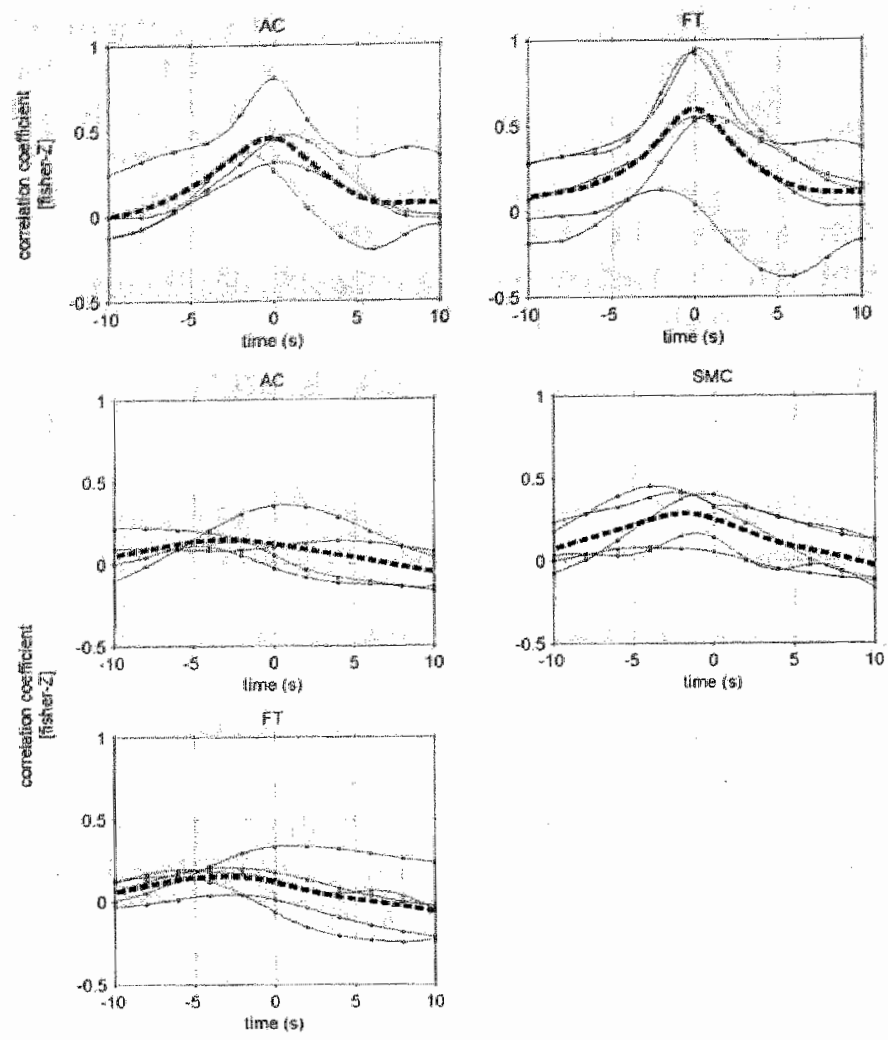

Figure 3.4. Upper row: Lag-correlation values. Correlations for each step (-10 to $10 \mathrm{TRS}$ around onset of button press) are calculated between the sensorimotor component activity timecourse and the auditory $(\mathrm{AC})$, and the fronto-temporal (FT) component. Middle and bottom row: Lag-correlation values. Correlations between the auditory, sensorimotor and fronto-temporal components and the convolved sequence of button presses. Broken line $=$ average of lagcorrelations; 0 correlation coefficient of true data point.

\section{Exploring additional areas}

The sICA approach allows for investigation of other connectivity patterns of areas within a single decomposition. Here, the right middle temporal gyrus (MTG) was investigated, which has been found active during auditory hallucinations in a few case studies (Lennox et al., 1999; Shergill et al., 2005 ). From each decomposition maximally one component was selected based on a spatial template of right MTG, which was generated from anatomical landmarks. Four of the five patients in the present study showed one separate component comprising suprathreshold voxels in right MTG, where the eventrelated average indicated a transient increase of activity only at the onset of the hallucinations. In contrast to other reports, there was no clear indication of a systematic lag difference (figure 3.5 ). 


\section{Chapter 3}

\section{Discussion}

Analysing the functional connectivity of AVH revealed the coactivation of sensory-specific and higher-order systems in the experience of the hallucinations. The sensory-specific network comprises primary auditory cortex, of which Heschl's gyrus is an anatomical landmark (Rademacher et al, 2001). A small number of previous studies has reported activity in primary auditory cortex during AVH in the dominant hemisphere (Dierks et al., 1999; Lennox et al., 2000). Our findings extend previous reports with activity during AVH in bilateral primary and secondary auditory cortex. Primary auditory cortex is not activated during auditory imagery (Shergill et al., 2000b; Halpern \& Zatorre, 1999). Therefore, activity in primary auditory cortex during hallucinations may be of a pathological nature, which may reflect impairments in left as well as right early auditory processing (McKay et al., 2000; Wible et al., 2001).

The higher-order network comprises areas in inferior frontal, superior and middle temporal gyrus, and medial frontal and inferior parietal areas, mainly but not solely in the left hemisphere. These findings are consistent with other reports (Dierks et al., 1999; Shergill et al, 2000a), and confirm that SICA is able to extract hallucination-related networks of activity. These areas are associated with language processing (Hickok \& Poeppel, 2000), which is presumed to be strongly lateralized to the dominant hemisphere in most right-handed healthy subjects, as well as with the monitoring of internally generated speech events (McGuire et al., 1996a).. Language processing and monitoring have been proposed to be associated with AVH in schizophrenia (Cahill \& Frith, 1996; Hoffman \& McGlashan, 1993; Stephane et al., 2001). The strong left lateralization of the fronto-temporal components contradicts the proposition that psychotic symptoms may arise from a decreased lateralization of language areas (Crow, 1997). At the same time, the finding that frontal and temporal areas were represented within a single component indicates a strong functional coupling between these areas, which seems to contradict findings of decreased fronto-temporal connectivity in schizophrenia (Lawrie et al., 2002). However, the strong fronto-temporal coupling during the experience of hallucinations may be state-related, whereas the associated decreased fronto-temporal connectivity may represent more stable, trait-related aspects of schizophrenia, such as abnormalities in fronto-temporal white matter connectivity (Hubl et al,, 2004).

The right MTG has been associated with detecting changes in the sensory environment (Downar et al., 2000), and was found to be activated at the onset of the hallucination events in the current study. When using the component of interest-activity timecourse of SMC and $\mathrm{AC}$ as a reference for computing lagdifferences no systematic difference in time shifts between any of these components were found. These findings are in contrast with case-reports of increased activity in right MTG several seconds prior to the onset of button 


\section{Functional connectivity of AVH}

Table 3.2. Correlation coefficients between hemodynamically conwolved sequence of button presses and component of interest-activity timecourses. COI = component of interest; $\mathrm{AC}=$ auditory component; $\mathrm{FT}=$ fronto-temporal component: $\mathrm{SMC}=$ sensorimotor component.

\begin{tabular}{cccc}
\hline Patient & \multicolumn{4}{c}{ COI } \\
\cline { 2 - 4 } & $\mathrm{AC}$ & $\mathrm{FT}$ & $\mathrm{SMC}$ \\
\hline 1 & 0.06 & -0.06 & $0.38^{*}$ \\
2 & $0.35^{*}$ & $0.18^{*}$ & $0.33^{*}$ \\
3 & 0.10 & $0.33^{*}$ & $0.39^{*}$ \\
4 & & $0.14^{*}$ & $0.13^{*}$ \\
5 & -0.02 & 0.02 & 0.06 \\
\hline
\end{tabular}

Notes: " $p<0.05$
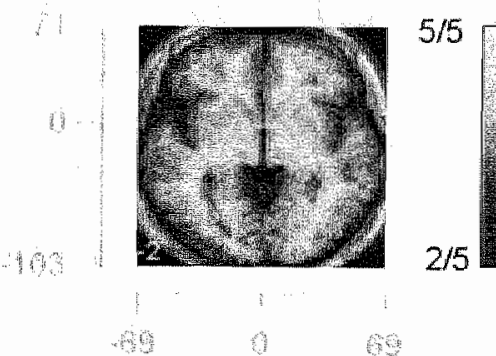

S5

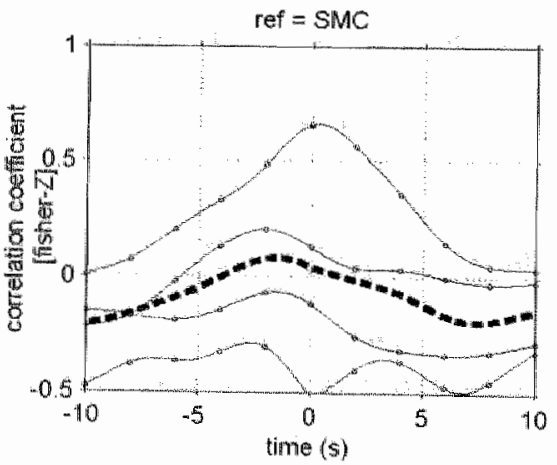

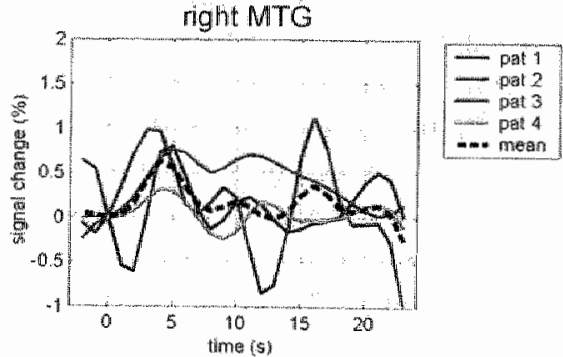

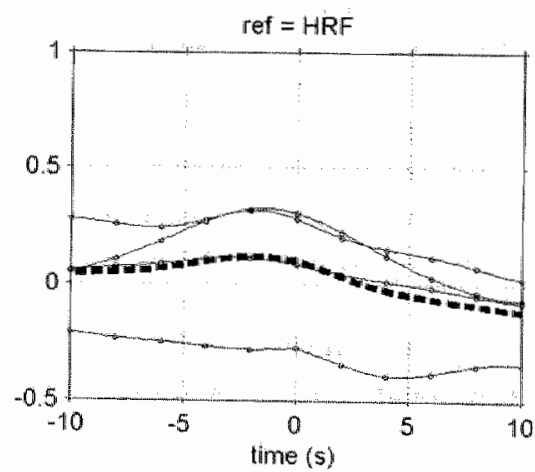

Figure 3.5. Right middle temporal gyrus (MTG). Upper row: Spatial map and eventrelated average of right $\mathrm{MTG}$ during hallucinations. Bottom row: lagcorrelations between right MTG and the sequence of button presses (ref = HRF) and the sensorimotor component ( $r e f=S M C$ ). For color version of upper row, see page 139. 


\section{Chapter 3}

Table 3.3. Correlation coefficients between hemodynamically convolved sequence of button presses and component of interest-activity himecourses. rMiG $=$ right middle tenporal gyrus; $\mathrm{PCC}=$ posterior cingulate component.

\begin{tabular}{c|cccc}
\hline Patient & 1 & 2 & 3 & 4 \\
\hline rMTG & $-0.29^{*}$ & $0.30^{*}$ & 0.08 & $0.27^{*}$ \\
\hline
\end{tabular}

Notes: $\mathrm{p}<0.05$

presses (Lennox et al., 1999; Shergill et al., 2005), and suggest that, on average, the neural activity related to the generation and experience of hallucinations occurs synchronously in all areas. However, it should be noted that we did not use specialized methods for the detection of differences in onset latencies in sub-TR resolution (e.g., Henson et al., 2002), which need to be investigated in future studies. In addition, sICA was restricted to cortical areas, which thereby ignores potential infuences from subcortical nuclei such as the thalamus, which may play an important role in schizophrenia (Andreasen, 1999).

Spatial ICA has been used to analyse functional data from healthy subjects when they monitored and responded to short sentences (Gu et al., 2001). The resulting decompositions revealed bilateral auditory cortex and language areas including inferior frontal and superior temporal gyrus in a single component. The separation of auditory and language areas into separate components during hallucinations may indicate a different functional coupling of these areas, reflecting a qualitative as well as neurophysiological difference in perceiving hallucinations, with respect to normally perceived sentences. While the functionally specialized areas in schizophrenia may still perform their associated functions, the communication between these areas, or networks thereof, may be impaired (Andreasen, 1999; Friston, 1998).

It should be noted that these findings do not verify the functional roles of these networks of areas in the experience of AVH. At the same time, anatomical differences in temporal (including Heschl's gyrus) and frontal gray matter (Gaser et al., 2004; Hirayasu et al., 2002) may affect the functional localization of specific neural networks, in comparison to healthy controls. Using connectivity analyses that incorporate knowledge of functional specialization within the same subjects (Hampson et al., 2002) could perhaps reveal more insight into this issue.

Our findings indicate that the functional anatomy underlying $\mathrm{AVH}$ can be parcellated into at least two different neural networks, which can be functionally distinguished as an early sensory and a language or monitoring network. The use of independent component analysis allows for the exploration of additional networks of areas, even when the temporal profile of these areas deviates from the ideal response model, as indicated by the sequence of button presses. 
Chapter 4

Components of target and distractor processing using self-organizing group-level ICA 


\section{Abstract}

Brain mechanisms of target and distractor detection are commonly investigated using oddball tasks, where participants identify a rare target stimulus from a train of equally rare but task-irrelevant distractor and frequent standard stimuli. We applied a data-driven analysis based on self-organizing group independent component analysis (sogICA) to fMRI data from oddball paradigm. This method is particularly suited to the investigation of the underlying functional connectivity and does not rely on a predefined model of the experiment, which helps to find unexpected activity pattern.

We found clusters of components for anterior and posterior cingulate and inferior parietal cortex, insula, and left and right posterior parietal cortex and frontal eye fields consistently across all participants. Independent components of parietal-temporal and ventral prefrontal areas for distractor processing, as well as primary visual cortex and of sensorimotor cortex exhibited more intersubject variability and were distributed across multiple clusters. Using smaller cluster sizes ( 8 and 7 ) the clustering of these components improved (smaller intra-cluster distances). Event-related averages indicated a categorization of the clusters into four groups: 1) target, but not distractor-related activity (insula, left parietal and frontal and sensorimotor cortex), 2) target and distractor-related activity (right parietal and frontal), 3) distractor but not target-related activity (cingulate and inferior parietal, and parieto-temporal and ventral prefrontal areas), and 4) no preference for either target or distractor (primary visual cortex). Our results show a parcellation of the target detection network, which is supported by other studies. At the same time, our results complement conventional results by showing activity in cingulate and inferior parietal areas within the same component related to distractors. SogICA can thus be used to probe functional networks of target detection at the group-level. 


\section{Introduction}

The detection of rare, meaningful events relies on the orchestration of several cognitive processes, including visual attention, working memory and stimulus categorization. Target detection is often investigated using the oddball task, where a target with low probability of accurrence is randomly presented within a train of standard, non-meaningful stimuli. The subject is required to detect the target by pressing a button or silently counting the amount of detected targets. In addition to the classical oddball task a three-stimulus oddball task has been

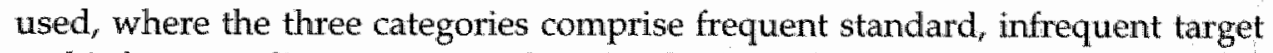
and infrequent distactor or novelty stimuli. The subject is instructed to respond only to target stimuli. The oddball paradigm has been often used in eventrelated potential (ERP) studies, where target and distractor stimuli elicit spatiotemporally different responses, termed the fronto-central $\mathrm{P} 3 \mathrm{a}$ and posterior $\mathrm{P} 3 \mathrm{~b}$ response respectively. The ERP amplitudes are also differentially modulated, with contextual sallience enhancing the frontal and task-relevance enhancing the posterior component (Goldstein et al., 2002; Gaeta et al., 2003).

Functional magnetic resonance imaging (fMRI) studies have demonstrated that target detection activates a network of areas around the Sylvian fissure, including the supramarginal gyrus, inferior and middle frontal gyrus and the insula, and midline areas, including the anterior and posterior cingulate and the supplementary motor area (Linden et al., 1999). Brain activity related to distractor stimuli, however, has been less consistent across studies. Using simple visual stimuli for all event types Bledowski et al. (2004) reported increased activity for distractor, compared to target stimuli in left prefrontal cortex, superior parietal cortex and precentral gyrus.

Many fMRI studies that used the oddball task investigated localized brain responses to the task. In this study, we attempted to probe components of functional connectivity related to target detection and distractor processing. Functional connectivity is operationally defined as the correlation between the timecourses of two spatially segregated areas (Friston, 1996), and is conceptually based on the notion that the brain comprises functionally specialized neural networks that may communicate during task performance. Spatial independent component analysis (sICA: Mckeown et al., 1998b) can be used to investigate whole-brain functional connectivity and probe multiple distinct networks simultaneously. SICA models the observed data (the voxel timecourses) as a linear combination of spatially independent sources. Components are decomposed without prior knowledge about the temporal. profile of brain activity. The assumption of spatial independence requires that the spatial components have non-normal probability distributions, while the component timecourses can be correlated to one another. This approach may be suitable for analysing functional. connectivity patterns in a cognitive task like the addball paradigm, because the associated cognitive systems are assumed to 


\section{Components of target processing using group ICA}

have spatial localization, albeit possessing a distributed pattern (e.g., anteriorposterior or left-right), while task performance requires the cooperation of these cognitive systems, which means that the associated timecourses are bound to be correlated to some degree. The degree of connectivity is reflected by the component values of each map.

SICA has been used to probe and characterize components of functional connectivity in a variety of situations, including complex naturalistic settings (Bartels \& Zeki, 2004), auditory responses in schizophrenia (Calhoun et al., 2004), and during rest (Van de Ven et al., 2004; see chapter one of this thesis). White many ICA applications were inherently based on single-subject analyses, there has been a recent increase in the development of multi-subject applications. Most of these developments focused on some combination of (preprocessed) functional data prior to ICA decomposition, where the concatenation of the data across subjects seems to provide the most reliable results (Calhoun et al., 2001a), even when a component is not present in all subjects (Schmithorst \& Holland, 2004). However, an alternative approach would consist in combining components from single-subject decompositions a posteriori, but in a way that reduces investigator subjectivity in selecting and grouping the components. A candidate for this approach is some form of cluster analysis that searches for similarity of components in the subject space. Clustering of independent components can be done using componentdescriptive parameters (Van de Ven et al., 2002), such as spatial structure or distributions (Formisano et al., 2002), or task frequency (Moritz et al., 2003). Esposito et al. (2005) suggested a different approach, where independent components of single-subject decompositions are grouped according to spatial or temporal information using a self-organizing grouping approach that is based on hierarchical cluster analysis (Himberg et al., 2004). This approach retains the individual decompositions and thereby circumvents potential errors in the decompositions that may be due to the combination of individual datasets into a preprocessed, aggregate dataset.

In this study, we probed the functional connectivity components of target detection and the encounter of rare, irrelevant stimuli, using self-organizing grouping ICA (soglCA: Esposito et al, 2005) in an attempt to unravel commonalities and differences between neural networks that underlie the processing of these two classes of stimuli. The association of a component to either of the two stimulus categories was investigated using event-related averages of the averaged functional timecourses of each subject.

\section{Methods}

\section{Subjects}

Functional imaging datasets of nine subjects were selected from an earlier study ([Bledowski et al., 2004] 6 males; mean age (S.D.) $=29.4$ (5.7) years; all were 


\section{Chapter 4}

right-handed). None of the subjects had a history of neurological or psychiatric disorders. After the study was explained all subjects gave written informed consent to participate in the study. The study was approved by the local ethics committee.

\section{Design}

A three-stimulus visual oddball paradigm was used, where the stimulus categories comprised standard, target and distractor stimuli. The standard stimulus (blue circle, $1.53^{\circ}$ ) had a probability of occurrence of 0.9 , while the target stimulus (blue circle, $1.38^{\circ}$ ), as well as the distractor stimulus (blue square, $1.36^{\circ}$ ), each had a probability of 0.05 to occur. The stimuli were presented to the subject in a random order, once every $2 \mathrm{~s}$ for $75 \mathrm{~ms}$. The subject was required to respond via button press to the target stimulus only. Within each functional run 350 stimuli were presented. A schematic display of the design is shown in figure 4.1 .

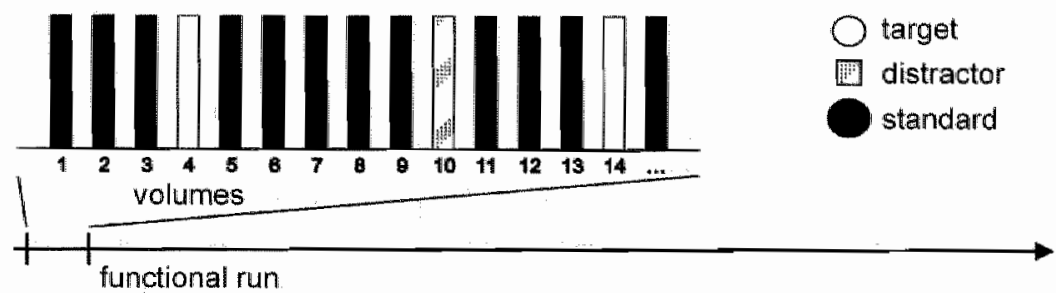

Figure 4.1. Schematic representation of the three-stimulus oddball task. During each volume a stimulus (standard, target or distractor) is presented.

\section{Imaging parameters}

All functional and anatomical images were acquired using a $1.5 \mathrm{~T}$ Siemens Vision MR Tomograph (Erlangen, Germany). The blood-oxygen-level dependent (BOLD) signal was measured using a gradient-echo echo-planarimaging (EPI) sequence. For the functional images, 16 axial slices were obtained for each volume (repetition time[TR]/echo time[TE] $=2000 / 60 \mathrm{~ms}$; voxel size $=$ $3.6 \times 3.6 \times 5.0 \mathrm{~mm}^{3}$; flip angle $=90^{\circ}$; field of view $[\mathrm{FoV}]=230 \times 230 \mathrm{~mm}^{2}$ ), and each functional run contained 360 volumes. During the first ten volumes no stimulus was presented, the onset of each proceeding volume triggered the onset of a stimulus. A three-dimensional anatomical image was acquired in the same session for each subject (matrix size $=256 \times 256 \mathrm{~mm}^{2}$; voxel size $=2.0 \times 1.0$ $\left.\times 1.0 \mathrm{~mm}^{3}\right)$, which lasted about five minutes.

\section{Preprocessing}

The first 4 volumes of each functional dataset were discarded because of saturation effects. The functional datasets were then corrected for inter-slice timing differences and scaled and resampled to Talairach space (Talairach and 
Tournoux, 1988) with a voxel size of $3 \times 3 \times 3 \mathrm{~mm}^{3}$ using the BrainVoyager 4.8 analysis software ( $w$ ww brainvoyager.com). The rescaled functional datasets were spatially smoothed using a Gaussian kernel of full-width-at-halfmaximum [FWHM] of $6 \mathrm{~mm}$, and temporally filtered using linear trend removal and high-pass filtering of 5 cycles per session $(\approx 0.007 \mathrm{~Hz})$. The anatomical images of all nine subjects were averaged, and a white matter mask was created from this average image. The white matter mask was used to exclude the marked voxels from further analysis. This procedure was done to attenuate computational load, while keeping the subspaces of selected voxels for analysis the same across subjects. In addition, restricting the decomposition to grey matter voxels improves the decomposition significantly (Formisano et al, 2004).

\section{Self-organizing grouping ICA (sogICA)}

The preprocessed functional datasets were imported and analyzed in Matlab 6 (MathWorks Inc., Mass.), using software described in Himberg et al. (2004) and Esposito et al. (2005). The sogICA framework analyses individually decomposed datasets on a group level by clustering independent components in the subject space, using spatial correlation as similarity measure. This approach preserves subject specific information, and provides the investigator with a measure of spatial similarity of components of interest. Regardless of the cluster size, all subjects are considered for clustering and each subject contributes maximally one component to a cluster. Infomax, an ICA algorithm that is based on a gradient-descent learning algorithm (Bell \& Sejnowski, 1995), was used to decompose each functional dataset into a set of 60 independent components. The initial learning rate was set to 0.0001 , while the batch size was set to 10. Prior to the ICA decomposition, the initial dimensions (i.e., number of timepoints) of the functional dataset were reduced to 60 using principal component analysis. After decomposition, the map values of each component map were Z-scored (McKeown et al., 1998a,b). Afterwards, a spatial correlation matrix (Pearson's product-moment correlation: $\mathrm{CC}$ ) was generated from all components, so that $\mathrm{CC}$ is of size $\mathrm{m} \times \mathrm{m}(\mathrm{m}=$ subjects $\times$ size of decomposition, in this case, $m=9 \times 60=540$ ), and $\mathrm{cc}_{i j}$ is the spatial correlation between the $Z$ scored components $I C_{1}$ and $I C_{j}\left(I C_{k,}, v=1,2, \ldots V ; V\right.$ is the number of voxels). The correlation matrix $\mathrm{CC}$ thus indicated the spatial similarity of the distribution of voxel values between component maps, and was used as the similarity matrix for hierarchical clustering. It should be noted that the sogICA framework allows the similarity matrix to be a combination of spatial and temporal information from the ICA decompositions (Esposito et al., 2005). We chose to use only spatial information because the timing of presentation and correct detection of target stimuli varied across subjects. The similarity matrix was then transformed into a dissimilarity matrix (DM) by

$$
\mathrm{DM}=\sqrt{1-\|C C\|}
$$




\section{Chapter 4}

which was used as a "spatial distance" matrix for the input of the hierarchical clustering step (Himberg et al., 2004). Then, clusters of components were generated as follows. In the initial step, the threshold for the intra-cluster distance was set to zero, and was subsequently increased following a step-wise change from 0 to 1 . The size of the step-wise change was set to 0.001 , but can be set as fine as the minimum distance between different components in the whole space of components (Esposito et al., 2005). At each step, a new cluster was generated when the intra-cluster distances were smaller than the current threshold. Each subject could contribute a component to a cluster maximally once. After a cluster was generated, the clustered components were excluded from successive steps.

The sogICA results are visualized in two ways. The dissimilarity matrix can be visualized by a 2-dimensional (2-D) projection of the intra-cluster distances, using multidimensional scaling-related methods (Torgerson, 1952). The plotted Euclidean distances of the cluster members will be slightly distorted with respect to the dissimilarities, but the general distribution of the plot will tend to capture the main structure of the comparative similarities (Esposito et al., 2005; see figure 4.3). In other words, a cluster with high spatial correspondence between the cluster members (i.e., small intra-cluster distances) will be closely positioned to each other within the 2-D space. Clusters with larger intra-cluster distances will show a more dispersed pattern in the 2-D space.

Cluster "group" components were calculated as random effects maps. The random effects statistic for each voxel was calculated as the mean ICA Z-value of that voxel across the individual maps divided by its standard error, resulting in a t-statistic, which was converted to a $\mathrm{z}$-statistic. The resulting map of $\mathrm{z}$ values was visualized using a threshold of $z=3.1$ ( $p<0.001$, one-sided), and a minimum number of 6 touching suprathreshold voxels. A lower-case $z$ is used for reference to the random effect, or cluster "group" map results, while an upper-case $Z$ is used for reference to the ICA voxel values for the individual component maps.

The cluster size was set to nine components. The clusters can be inspected using a visual display of the dispersion of each item within a cluster, and by examining the mean, minimum and maximum intra-cluster distances of each cluster (Esposito et al., 2005). Clusters of spatially similar components show small intra-cluster distances between the cluster mean and the components.

After sogICA was performed, we chose components and clusters of interest according to 1$)$ the 10 clusters with the lowest mean intra-cluster distances, and 2) spatial templates of areas of interest, which included bilateral insula, inferior parietal cortex, dorsolateral prefrontal cortex, middle/inferior temporal cortex, posterior cingulate cortex and primary visual cortex. The search strategies were used independently in order to complement each other.

For each cluster, group event-related averages (and standard errors of the mean) were generated from the event-related averages obtained from the raw 


\section{Components of target processing using group ICA}

timecourse dato of the individual components for the target and distractor stimuli. For the farget stimulus only correctly identified trials were used for event-related averaging. For visualization the group event-related averages were interpolated to a resolution of 1 second.

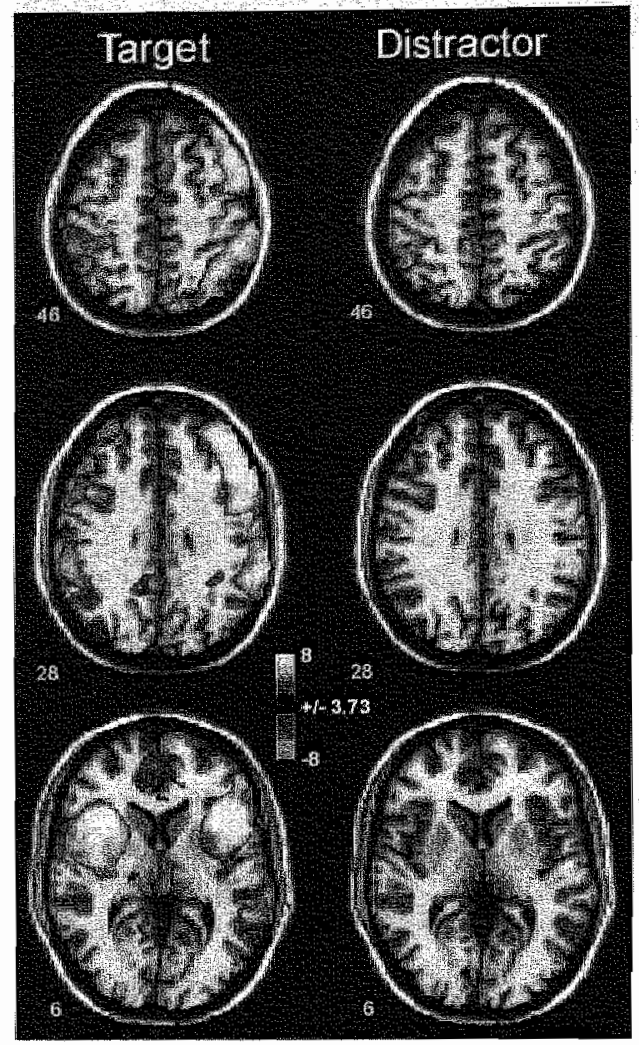

Fingure 42. Transwerse maps of the main effects of target and distractor processing. Maps are thresholded at $p<0.0002$ (falsediscovery rate of $0.001 ; \mathrm{t}(3202)=3.73)$. For target processing, activity occurred in many areas in parietal, frontal and temporal cortex. For distractor processing, activity was mostly lateralized to the left hemisphere. In images, left hemisphere is presented on the left. For color version, see page 140.

\section{Results}

\section{Behavioural results}

The nine subjects correctly identified $85.9 \%$ of the targets $($ S.D. $=9.6 \%)$. Figure 4.2 shows the main effect results of the multiple regression analysis of target and distractor processing thresholded using a false discovery rate $\mathrm{q}=0.001$ (Genovese et al., 2002). The main purpose of this figure is to serve as a reference for the sogICA results.

\section{SogICA results}

Using the search strategies of spatial templates we found seven clusters, of which three ranked within the 10 lowest intra-cluster distances. Table 4.1 lists 


\section{Chapter 4}

the mean and range of the intra-cluster distances for these seven clusters. Figure 4.3 depicts the dispersion of the cluster members for four of the seven chosen clusters. The random effect maps, or cluster "group" maps, of four of the seven chosen cllusters are shown in Figure 4.4. Table 4.2 lists the peak $\mathbb{Z}$-values of the group maps.

\section{Spatial maps}

All nine components of the chosen cluster with the smallest mean intra-cluster distance comprised suprathreshold $z$-values mostly in posterior cingulate cortex, but also in anterior cingulate and bilateral inferior parietal cortex ( $\mathrm{pCC}$ cluster). Figure 4.2 indicated a potential outlier within the pCC cluster, i.e. one cluster member (S7) is spatially displaced with respect to the other cluster members. Close inspection of this particular member's spatial layout revealed that, in addition to posterior cingulate and bilateral inferior parietal cortex, a large area of suprathreshold Z-values was located near the anterior cingulate cortex. The second cluster contained areas of high $z$-values (random effects map as well as individual component maps) in right posterior parietal cortex and frontal eye field (rPPC-FEF cluster). All nine individual components showed a right lateralization of high Z-values. The third cluster contained suprathreshold voxels in bilateral insular areas, frontal operculum and inferior frontal gyri (billNS cluster). Inspection of individual maps revealed additional areas of suprathreshold Z-values in inferior frontal and frontal medial areas in some, but not all, maps. The fourth cluster contained high Z-values in mostly bilateral middle frontal gyrus, but also in parietal areas in the random effects map (bilPFC). Inspection of the individual maps indicated some variability in spatial location of suprathreshold voxel values. Two spatial components contained suprathreshold voxel values in right posterior and dorsal frontal cortical areas. In the other components areas of high functional connectivity were found in both the left and right hemisphere. For one component, connectivity clusters were found in bilateral dorsal frontal areas, including frontal eye fields, but not in parietal areas. The fifth cluster comprised areas in left parietal and temporal cortex, as well as dorsal and ventral prefrontal regions (PT-VPF).

\section{Group event-related averages}

Group event-related averages for the four clusters are calculated from the event-related averages of the individual components within each cluster. For each component map the voxel timecourses were extracted from voxels with positive suprathreshold Z-values. Figure 4.4 depicts the group event-related averages and standard error of the mean for the target and distractor stimuli.

The billNS and bilPFC clusters showed event-related BOLD signal increases for the target stimulus, but not for the distractor stimulus. Right PPCFEF showed a large increase of BOLD signal for the target stimulus and a small initial increase for the distractor stimulus. The PCC cluster showed a decrease of the BOLD signal for the target stimulus, and an increase of the BOLD signal 
Components of target processing using group ICA

for the distractor stimulus. The PT-VPF cluster showed increased activity for the distractor, but not the target stimulus.

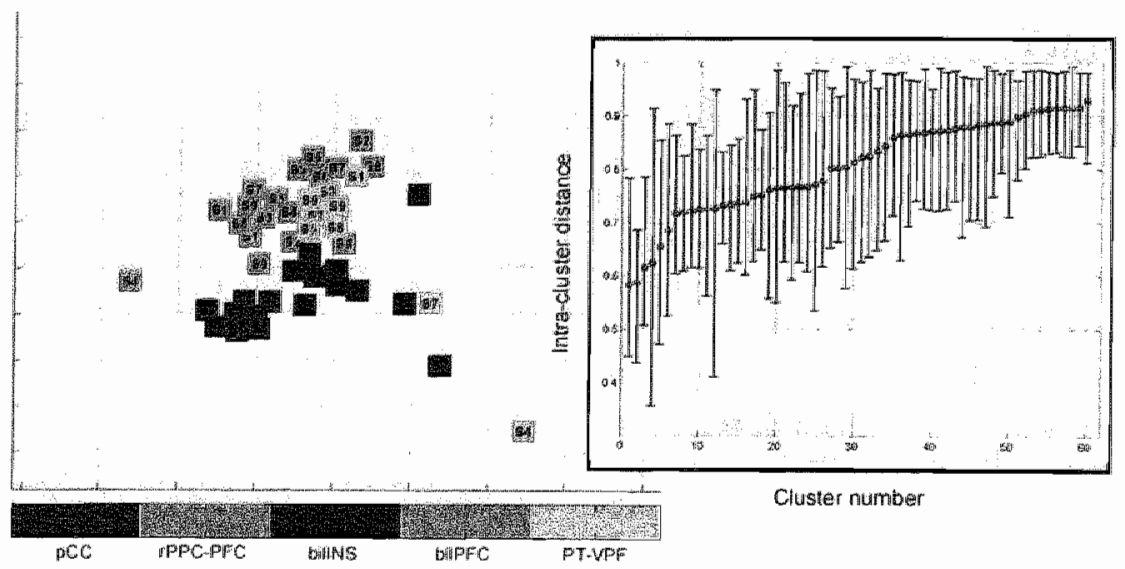

Figure 4.3.

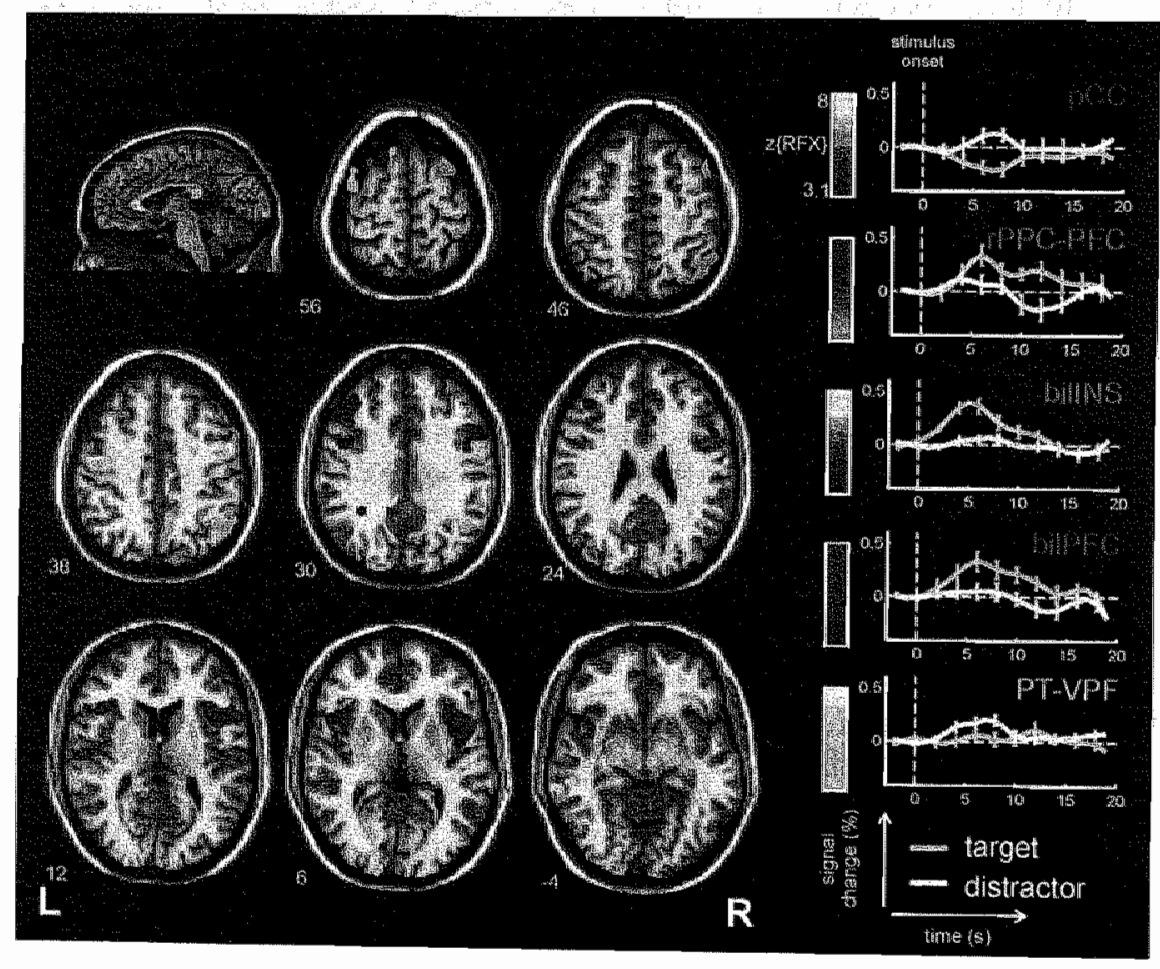

Figure 4.4. 


\section{Chapter 4}

Figure 4.3. Intra-cluster distances of $\mathrm{pCC}, \mathrm{PPC}-\mathrm{PFC}$, biliNS, bilPFC and PT-VPF Left Scatter plot shows projected intra-cluster distances for the clusters of posterior cingulate cortex ( $\mathrm{pCC}$ : red), right posterior parietal cortex and frontal eye field (rightPPC-FEF: green), bilateral insula (billNS: dark blue), bilateral prefrontal cortex (biliPFC: purple), and parietal-temporal and ventral prefrontal cortex (PT-VPF: light blue). Coding of cluster member: $\mathrm{S1}=$ subject 1 . Right: minimum, mean and maximum intra-cluster distance for all clusters. The portrayed clusters in the scatter plot are marked in red. For color version, see page 140 .

Figure 4.4. Spatial group maps and event-related averages of 5 clusters of interest. Transverse views ( $Z$-coordinate in yellow) of the random effects maps (thresholded at $z=3.1, p<0.001$, one-sided). Cluster size is nine components per cluster. Left-upper inset: Z-planes of presented images on coronal view of template brain (blue lines). Right column: event-related averages $(t /-1$ standard error), obtained by averaging the component averages, and interpolated to $1 \mathrm{~s}$. For color version, see page 141.

\section{Additional clusters}

We also searched for clusters of sensorimotor (SMC) and primary visual cortex (PVC) (see Table 4.3 for Talairach coordinates). Components for these areas did not cluster well, which is represented by relatively large intra-cluster distances. Components of SMC were clustered into two different clusters, together with spatial maps that contained high $Z$-values in dorsal areas, but which were not likely related to the neurophysiological processes of button presses. We therefore performed two additional clustering runs with cluster sizes of respectively eight and seven subjects per cluster. Note that in the two additional runs the complete datasets were used (60 components $\times 9$ subjects), and that each subject contributed maximally one component to each cluster. Clustering for these areas improved with decreasing cluster size (decrease in minimum, mean and maximum intra-cluster distances, see figure 4.5 and table 4.3). Spatial maps and event-related averages of SMC and PVC (cluster size $=7$ ) are shown in figure 4.6. Left SMC revealed increased activity for the target, but not for the distractor stimulus, which is expected because it reflects the metabolic activity related to pressing the button when a target is detected. PVC showed no change from baseline for either of the stimulus categories.

While right middle temporal gyrus (rMTG) was clearly found activated during target processing using multiple linear regression (see figure 4.2), sogICA did not find an rMTG cluster using cluster sizes of 9,8 or 7 components. To verify whether rMTG was present in the individual decompositions, we tried to find a spatial component with suprathreshold voxel values in the right MTG for each subject separately. The decompositions of six subjects comprised one rMTG component that was not part of any of the reported clusters of the clustering size of 9 components, which indicates that rMTG is considered as a separate spatial source (using a decomposition size of 60 components). 


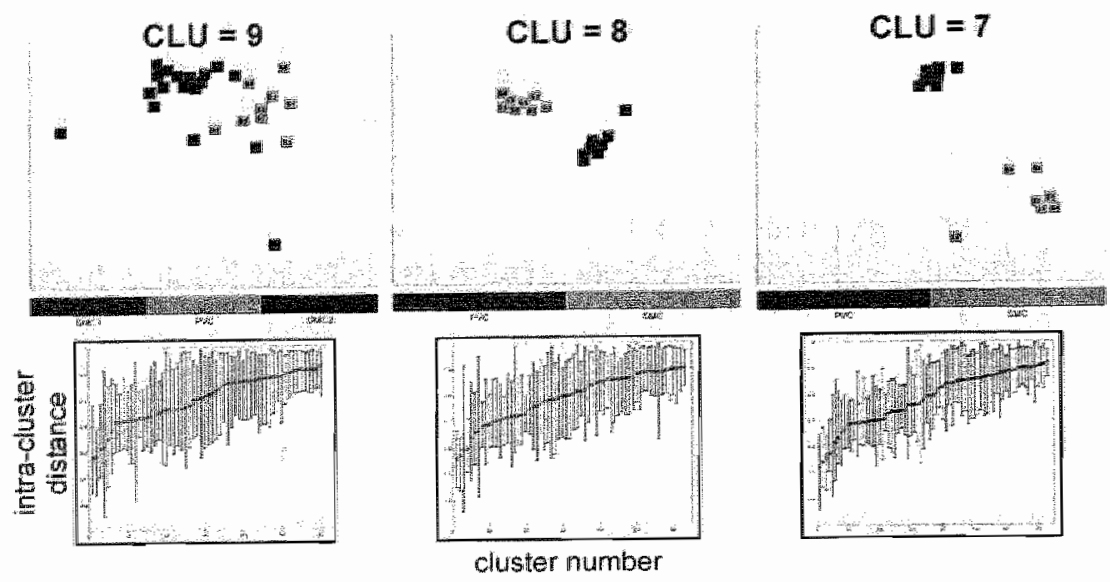

Figure 4.5. Intra-cluster distances of SMC and PVC across three different cluster sizes. For cluster size of 9, SMC components were grouped in two separate clusters, together with components that contained high $Z$-values in similar areas of the brain, but that were not related to the button press. Decreasing the cluster sizes increased the spatial similarity within the clusters (smaller cluster distances, as represented by less dispersion within the plot). For color version, see page 141.

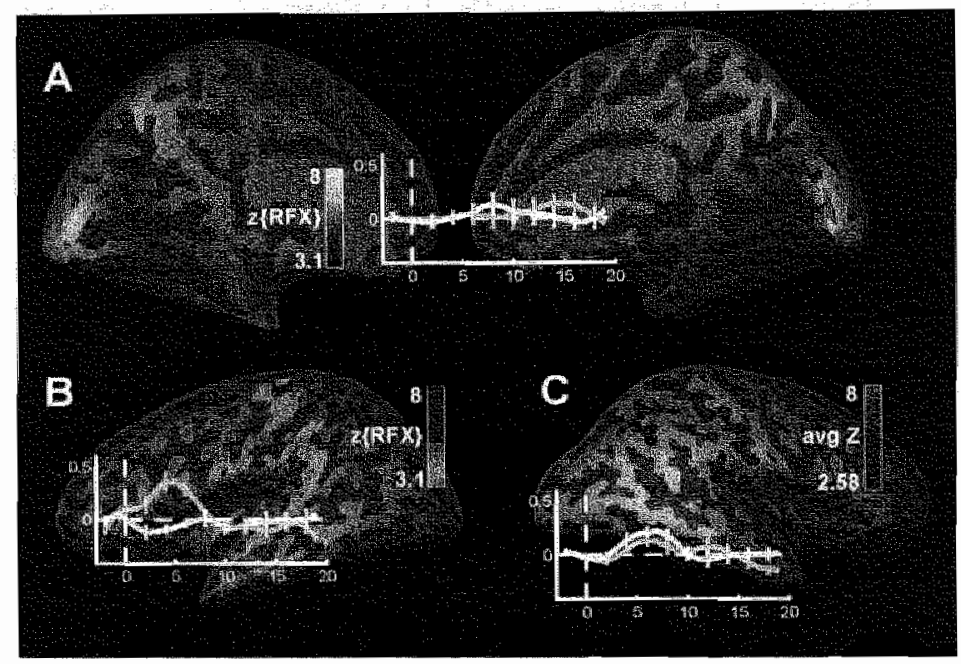

Figure 4.6. Additional spatial maps and event-related averages of target and distractor stimuli. A: Primary visual cortex (PVC) cluster of cluster size $=7 . \mathrm{B}$ : Sensorimotor cortex (SMC) cluster of cluster size $=7 . \mathrm{SMC}$ shows increased activity for the target stimulus, related to the button presses. PVC and SMC are "random effects" group maps, thresholded at $\mathrm{z}=3.1$. $\mathrm{C}$ : right middle temporal gyrus (rMTG). Average spatial Z-map (thresholded at $Z=2.58$ ) of 6 subjects. Event-related averages show increases for the target and distractor stimulus. rMTG components were selected manually, not using sogICA. For color version, see page 142 . 


\section{Chapter 4}

Table 4.1. Intra-cluster distances. Mean and range of the intra-cluster distances of the seven selected clusters (see insets of figure 43 and 4.5). For cluster abbreviations, see text. Size $=$ number of cluster members (components).

\begin{tabular}{ccccc}
\hline Cluster & Size & Mean & \multicolumn{2}{c}{ Range } \\
\cline { 4 - 5 } & & & Min & Max \\
\hline PCC & 9 & 0.62 & 0.51 & 0.79 \\
rPPC-FEF & 9 & 0.72 & 0.61 & 0.86 \\
billNS & 9 & 0.73 & 0.61 & 0.85 \\
bIIPFC & 9 & 0.77 & 0.63 & 0.96 \\
PTI-VPF & 9 & 0.77 & 0.63 & 0.94 \\
\hline PYC & 7 & 0.69 & 0.58 & 0.78 \\
SMC & 7 & 0.72 & 0.66 & 0.80 \\
\hline
\end{tabular}

Examination of the individual components revealed some variability across the spatial maps, where three components comprised bilateral MTG. The group event-related average indicated increased activity for the target, as well as the distractor stimulus (figure 4.6.C). In the remaining three subjects no rMTG component was found.

\section{Discussion}

We analysed the functional imaging data of a three-stimulus oddball task of nine subjects using the sogICA framework to interpret the results on a group level. SogICA grouped the decompositions of individual datasets into clusters of nine subjects, where each subject contributed maximally one component to each cluster. Seven clusters of interest of spatial component maps were found, of which five had low to moderate intra-cluster distances, which indicated a high spatial correspondence across the cluster members.

Components comprising areas in billateral prefrontal cortex (bilPFC), insula and bilateral inferior frontal sulcus (bilINS), right parietal and frontal cortex (rPPC- PFC) and left sensorimotor cortex (SMC) showed increased BOLD signal activity for the target. These areas have been reported in many fMRI studies of target detection in the oddball task (Ardekani et al., 2002; Clark et al., 2000; Linden et al., 1999; Kiehl et al., 2001). Bilateral ventro-lateral PFC (including inferior frontal gyrus and frontal operculum) has been proposed to be involved in active memory retrieval (Cadoret et al., 2001). Right parietal and frontal areas showed an initial increase in activity for the target as well as distractor stimulus, but the signal dropped to baseline for the distractor stimulus, while the signal increased further for the target stimulus. These regions are part of a parietal-frontal network that has been associated with stimulus-driven and voluntary attentional shifts which are modulated by task relevance (Corbetta and Shulman, 2002; Hopfinger et al., 2002). Right parietal and frontal areas have 


\section{Components of target processing using group ICA}

Table 4,2. Talairach coordinates $(x, y, z)$ of peak $z$-values (random effects [RFX]) of cortical areas of the four clusters comprising nine components. Maps were thresholded at $2[R F X)=3.1$ ( $p \leq 0.001$ ) and a minimum cluster size of 10 voxels $(3 \times 3 \times 3$ min3). Size of clusters is reported in number of voxels. $\mathrm{ACC}=$ anterior cingulate cortex; $\mathrm{PCC} / \mathrm{PreC}=$ posterior cingulated cortex/precuneus; $\mathrm{PreCG}=$ precentral gyrus; $S F G=$ superior frontal gyrus; $M F G=$ middle frontal gyrus; IFG = inferior frontal gyrus; IPL = inferior parietal lobule; IPS = intra parietal sulcus; (a)MTG = (anterior) middle temporal gyrus; INS = insula; FO = frontal operculum; $\mathrm{MedFG}=$ medial frontal gyrus; $\mathrm{SMA}=$ supplementary motor area; $\mathrm{OG}=$ accipital gyrus.

\begin{tabular}{|c|c|c|c|c|c|c|c|}
\hline Cluster & Area & $L / R$ & $x$ & $y$ & $z$ & $z[\mathrm{RFX}\}$ & size \\
\hline \multicolumn{8}{|l|}{ pcc } \\
\hline & $\mathrm{ACC}$ & $L / R$ & -1. & 46 & 3 & 3.8 & 10 \\
\hline & PCC/PreC & $L / R$ & -10 & -56 & 24 & 5.8 & 753 \\
\hline & $\mathrm{PP} / \mathrm{MTG}$ & L & -46 & -68 & 24 & 5 & 101 \\
\hline & aMTG & $\mathrm{R}$ & 59 & -8 & -12 & 4.5 & 13 \\
\hline & IPL & $R$ & 47 & -62 & 24 & 3.5 & 13 \\
\hline \multicolumn{8}{|l|}{$\mathrm{MP} P C-\mathrm{HAS}^{\mathrm{B}}$} \\
\hline & PreCG & R & 53 & 10 & 39 & 3.8 & 35 \\
\hline & $\mathrm{SFG}$ & R & 26 & 49 & 21 & 3.8 & 14 \\
\hline & MFG & $\mathrm{R}$ & 26 & 7 & 57 & 4.6 & 35 \\
\hline & MedFG & $R$ & 5 & 19 & 48 & 3.6 & 11. \\
\hline 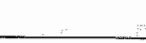 & $\mathrm{IPL}$ & $\mathrm{R}$ & 41 & -53 & 33 & 4.3 & 188 \\
\hline \multicolumn{8}{|l|}{ billNS } \\
\hline & IFG & $\mathrm{L}$ & -34 & 19 & 15 & 4.2 & 203 \\
\hline & $5 F G$ & $\mathrm{R}$ & 5 & 19 & 66 & 3.9 & 15 \\
\hline & INS/RO & L. & -34 & -17 & -3 & 3.8 & 13 \\
\hline & & $\mathrm{R}$ & 38 & 10 & 9 & 4.5 & 271 \\
\hline & MedFG/SMA & $\mathrm{L}_{4}$ & -4 & 13 & 36 & 3.6 & 12 \\
\hline \multicolumn{8}{|l|}{ bilPFC } \\
\hline & $\mathrm{MHG}$ & $L$ & -37 & 31 & 30 & 4.1 & 164 \\
\hline & & $\mathrm{R}$ & 44 & 31 & 21 & 3.3 & 12 \\
\hline & $\mathrm{IFG} / \mathrm{MFG}$ & $\mathrm{R}$ & 53 & 10 & 33 & 3.7 & 71 \\
\hline & IPS & L & -31 & -47 & 30 & 4.2 & 10 \\
\hline & OG & $\mathbb{L}_{3}$ & -28 & -80 & 24 & 3.8 & 10 \\
\hline \multicolumn{8}{|l|}{ PT-VPF } \\
\hline & STG & $\mathbb{L}$ & -10 & 22 & 54 & 3.7 & 11 \\
\hline & $\mathrm{MTG}$ & L & -40 & -5 & 57 & 4.1 & 36 \\
\hline & $\mathbb{I P C}$ & L. & -52 & -59 & 24 & 3.4 & 13 \\
\hline & $1 F G$ & L & -46 & 31 & 6 & 3.7 & 35 \\
\hline & & L. & -52 & 10 & 24 & 4.2 & 20 \\
\hline & MTG & $\mathrm{L}$ & -52 & -32 & -3 & 3.9 & 33 \\
\hline
\end{tabular}




\section{Chapter 4}

also been associated with sustained attention (Coull et al., 1998; Lawrence et al, 2003), showing initial increase of activity for infrequent stimuli (i.e., both target and distractor stimuli in the current study). Further processing, however, may then only be allocated to those stimuli identified as targets, resulting in a further increase of activity. Support for such an interpretation comes from single-cell recording studies of the frontal eye field (FEF), where spiking activity to target selection showed a similar two-step spiking activity pattern. After an initial increase of activity, regardless of stimulus relevance, spiking activity increased for target stimuli, and decreased for distractor stimuli (Bichot et al., 2001), which suggests the mapping of stimulus salience in FEF.

The cluster that contained anterior and posterior cingullate and bilateral inferior parietal cortex showed increased activity for the distractor, and decreased activity for the target stimulus. Increased activity in anterior cingulate cortex for the distractor stimulus was also reported by Clark et al. (2000). Posterior cingulate cortex and inferior parietal cortex may be associated with monitoring ongoing events that do not require the intentional allocation of processing resources (Shulman et al., 1997). The ongoing monitoring is inhibited or disrupted when resources are allocated for effortful, intentional processing, which results in a decrease of activity in the associated brain areas (Raichle et al., 2001; Greicius et al., 2003). Distractor stimuli, although drawing attentional resources reflexively, do not demand intentional processing, and therefore do not disrupt monitoring to a large extent. This is not the case for the target stimulus, which demands further processing, which is reflected by the inhibition of the ongoing monitoring system.

Activity specifically related to the distractor stimulus was found in a cluster of components that comprised areas of left parietal, medial and middle/inferior frontal and middle temporal gyrus. Left middlle/inferior frontal and parietal areas have been associated with retention of object-related ('what') information in working memory (Munk et al., 2002). Because the distractor stimulus differed geometrically from the standard and target stimuli, it is conceivable that a decision was reached by matching the distractor object to the stored target template. The target and standard stimuli belonged to the same object category and differed only marginally, which may have resulted in the recruitment of additional, or different comparison strategies for stimulus categorization.

The remaining two clusters (SMC and PVC) showed a high degree of spatial variability across the cluster members, which was reflected by the relatively high intra-cluster distances. When the cluster runs were redone with smaller cluster sizes the clustering for SMC and PVC improved markedly. The initial failure of the sogICA framework to cluster the SMC components may have been due to the presence of components with high Z-values in similar dorsal areas, but that were not related to the neurophysiological process associated with the button presses. 


\section{Components of target processing using group ICA}

Table 4.3. Talairach coordinates $(x, y, z)$ of peak $z$-values (random effects) of cortical areas of SMC and PVC dusters comprising. Cluster size $=7$ components. $\mathrm{LG}=$ lingual gyrus; $\mathrm{Cu} / \mathrm{CalcF}=\mathrm{Cuneus} / \mathrm{Calcarine}$ fissure; PostCG $=$ postcentral byrus.

\begin{tabular}{|c|c|c|c|c|c|c|c|}
\hline Cluster & Area & $\mathbb{L} / \mathrm{R}$ & $x$ & $y$ & $z$ & $\mathbb{Z} \mathbb{R P X}$ & Size \\
\hline \multicolumn{8}{|l|}{ PVE } \\
\hline \multirow[t]{3}{*}{. } & $\mathrm{LG}$ & L & -16 & -65 & -15 & 3.5 & 17 \\
\hline & Cu/CaleF & $\mathbb{R}$ & 5 & -77 & 18 & 4.4 & 266 \\
\hline & parahippocampal $\mathrm{G}$ & $\mathrm{R}$ & 23 & -47 & -6 & 3.7 & 11 \\
\hline \multicolumn{8}{|l|}{$S M C$} \\
\hline & PostcG & L & -55 & -23 & 48 & 4.8 & 233 \\
\hline & & R & 56 & -23 & 48 & 4 & 23 \\
\hline & Cerebellum & $\mathrm{R}$ & 23 & -41 & -21 & 4 & 16 \\
\hline
\end{tabular}

SogICA was not able to replicate the complete results of the multiple regression results, failing to identify a cluster of right middle temporal gyrus (rMTG). Systematic manual search for rMTG components within each subject revealed a large variability $\mathrm{rMTG}$ across subjects, where in three cases no such component was found. Thus, the null-result of sogICA indicated that rMTG was be a problematic component to obtain and cluster across subjects, rather than a shortcoming of the sogICA framework. Downar et al. (2000) showed that right middle temporal gyrus may be part of a network underlying the involuntary orienting of attention to changes in the sensory environment, which is in correspondence to our finding that rMTG responded to the target, as well as the distractor stimulus.

In all subjects the decompositions resulted in separate components for the right $\mathrm{PPC}$, with mostly right superior frontal gyrus also falling within the same component. This lateralization may indicate a differential contribution of right parietal-frontal areas to visual attention, as compared to the left hemisphere (Coull et al., 1998; Lawrence et al., 2003; Muggleton et al., 2001; O'Shea et al., 2004). The decomposition of left and right parietal-frontal areas into separate lateralized components was also observed for a functional connectivity study on the resting brain (i.e., when not performing any specific task) (Van de Ven et al., 2004). However, it should be noted that the separation may represent a faulty decomposition due to incorrect decomposition parameters (e.g., number of components, assumption of spatial independence), or a hemispheric difference in neuro-vascular coupling, rather than in functional anatomy (Mitra et al., 1997).

Previously, the sogICA framework was used to investigate the hemodynamic response to the presentation of a standard visual stimulus (Esposito et al., 2005). In the current study, we investigated hemodynamic responses to a complex visual task presented in an event-related design where multiple cognitive components are at play. While sogICA replicated to a large extent the findings of the conventional, hypothesis-driven analysis, the 
framework revealed additional information about the brain's responses to the task. First of all, the two analyses provided different kinds of information. The hypothesis-driven analysis indicated where in the brain the differential activity for either target or distractor stimuli was significant. ICA, on the other hand, indicated which brain areas showed the highest degree of shared information amongst one another regardless of the differential effects. While inherently different, these two approaches can be used complementary. Both analyses revealed areas for either the target stimulus (in sogICA cluster rPPC-PFC) or the distractor stimulus (cluster PT-VPF). However, sogICA also revealed components of right parietal and frontal areas that showed initial increases for both stimulus categories, which is not found with the conventional analyses. Also, a network of anterior and posterior cingulate, as well as bilateral inferior parietal cortex, was found, where activity for the distractor stimulus increased and activity for the target stimulus reversed sign. Hypothesis-driven analysis showed the target-related decrease of activity, but not the distractor-related increase.

The visualization techniques employed by the sogICA framework allow for the investigation of grouping of components across different subjects. The spatial similarity of components within a cluster can be inspected visually using multidimensional scaling plots, and quantitatively by comparing the intracluster distances. Cluster members of clusters that portray relatively large and variable intra-cluster distances (dispersion within the plot) can be viewed critically with respect to their spatial layout (and their temporal profile, if sogICA has clustered components according to some combination of spatial and temporal information), and potential outliers can be identified. To improve grouping, the clustering can be repeated with a smaller cluster size. Although this may lead to an improved clustering of more homogeneous components across subjects, this may not always be the case if similar spatial sources have different spatial configurations, perhaps due to profound inter-subject anatomical variability. This issue is of a more general concern, and techniques that can reduce the anatomical variability, for example by morphing the individual anatomical images to an anatomical template may prove to facilitate clustering in the sogICA framework.

The spatial independent components can be interpreted as maps of functional connectivity (Van de Ven et al., 2004; Yang \& Rajapakse, 2004), because the maps indicate a high amount of shared information on a timepointby-timepoint basis. Moreover, it may be possible to interpret these connectivity components in terms of functional specialization (Friston, 1996), where different specialized networks can interact given a specific task, which is reflected in the correlations between component timecourses.

In conclusion, application of the sogICA framework to oddball task functional data yielded connectivity patterns that were related to 1) either the detection of target or 2) the processing of distractor stimulus, 3) the processing of both target and distractor stimuli, and 4) neither target nor distractor. 
SogICA could replicate most, but not all, hypothesis-driven results across nine subjects, while at the same time reveal new insights into the hemodynamic responses related to target and distractor processing, which can be used to generate new hypotheses for further study.

\section{Acknowledgements}

This study was financially supported by a grant from the Alzheimer Research Initiative to D.P. a grant from the German Academic Exchange Service (D.A.A.D.), to V.G.V. and C.B. We thank Adriana Agrari for helpful discussion. 


\section{Chapter 5}

\section{Enhanced vividness of mental imagery as a trait marker of schizophrenia?}

Based on publication:

Sack, A.T., Van de Ven, V.G, Etschenberg, S., Schatz, D. \& Linden, D.E.J. (2005). Enhanced vividness of mental imagery as a trait marker of schizophrenia? Schizophrenia Bulletin, 1, 97-104. 


\section{Enhanced imagery vividness and hallucinations}

\section{Abstract}

We assessed the vividness of mental imagery in schizophrenic patients in the context of psychopathology and cognitive abilities. A questionnaire on the vividness of mental imagery (QMI) and a hallucination scale were administered to fifty patients with paranoid schizophrenia. The related perceptual and cognitive skills, general intelligence level, and psychomotor speed were measured as covariates with a battery of performance tests. All measures were statistically compared to a group of fifty age- and sex-matched healthy controls. The schizophrenic group obtained higher values both for vividness of imagery and occurrence of hallucinations. These differences were independent of general intelligence and psychomotor speed and did not correlate with individual psychopathology. The correlation between the hallucination and imagery scales themselves was very low. These results suggest that patients with schizophrenia experience a significantly higher vividness of mental imagery than healthy controls, which does not seem to be an effect of other group differences or individual psychopathology (such as frequency of hallucinations). Vividness of mental imagery might thus prove to be an independent trait marker of schizophrenia. 


\section{Chapter 5}

\section{Introduction}

Haliucinations have been widely investigated but still remain among the most puzzling psychopathological phenomena. Mental imagery resembles hallucinations in that mental images also have perceptual qualities and occur in the absence of appropriate stimuli. The difference between hallucinations and mental images lies in the possibility to control the perceptual experience. While hallucinations typically occur beyond intention and control (Bentall, 1990), mental images are actively generated by the subject and can thus be intentionally controlled. Subjects often describe their imagined experiences as echoes or reconstructions of former perceptual experiences. Imagery might aid cognitive functions in the respective sensory domain. It has, for example, been claimed that visual imagery ability correlates with visuospatial memory span (Kail, 1997).

Some authors suggested that hallucinations and vivid imagery are in some way related. Mintz and Alpert (1972) claimed that individuals who hallucinate also have very vivid images and a weak ability to distinguish real perception from imagery. Theoretical models suggested that hallucinations are misinterpreted mental images derived from internal sources of information (Cahill \& Frith, 1996; Horowitz, 1975). These images are incorrectly evaluated as arising from external sources, and appear as intrusions in the perceptual process. Accordingly, hallucinations would represent a failure of a reality monitoring system, which implies that internally generated experiences are erroneously ascribed to an external source. In addition, it has been proposed that hallucinations are not confined to psychiatric illness but that they might exist on a continuum between normalcy and psychopathology (Bentall, 1990).

Barrett (1993) and Barrett and Etheridge (1992) carried out several studies on the incidence of hallucinations in non-clinical populations. They revealed that nearly $50 \%$ of their subjects had hallucinatory experiences once a month. They observed that people with hallucinations had a more vivid imagery, but a worsened control of images in comparison to people who did not experience hallucinations. These and other reports suggest that non-clinical and pathological hallucinations share at least some features, and that mental imagery abilities might contribute to the experience and report of hallucinations in a variety of settings or populations.

However, the altered evaluation of mental images is not the only psychological mechanism that has been adduced to explain hallucinations. Fantasy proneness (Van de Ven \& Merckelbach, 2003) and other types of response bias (Bentall \& Slade, 1985a) have to be taken into account as well.

The link between hallucinations and mental imagery is even more controversial for clinical populations. For example, Brett and Starker (1977) and Starker and Jolin (1982) did not find more vivid imagery in hallucinating 


\section{Enhanced imagery vividness and hallucinations}

schizophrenic patients than controls. Chandiramani and Varma (1987) likewise found no significant differences in the vividness of imagery between hallucinating schizophrenic patients, non-hallucinating schizophrenic patients and normal controls. Böcker et al. (2000) tested the hypothesis that hallucinations result from a confusion of external and internal stimulus sources. They found no group differences in the mental imagery ability between the schizophrenic group and normal controls. However, for the hallucinating schizophrenic patients, the relative level of vividness of mental images was higher in the auditory modality, which was the modality in which most of the patients experienced hallucinations.

Several factors may have contributed to the inconsistency of results. Aleman et al. (1999) pointed out that objective and subjective mental imagery measures may yield contradictory results within the normal population. This distinction may hold true in some cases for the clinical population, as well. For example, Mintz and Alpert (1972) based their finding of an association between increased mental imagery and hallucinations on suggestion paradigms or selfreport measures, while Böcker et al. (2000) used a more objective mental imagery task to show that mental imagery performance did not differ between patients and controls. In addition, most studies did not incorporate measures sensitive to cognitive differences between or within patient and control groups.

In this study we investigated the vividness of mental imagery and hallucinations in a group of fifty schizophrenic patients compared to a group of fifty age- and sex-matched controls. Bett's Questionnaire upon mental imagery (QMI) was used as an operationalization of mental imagery. This questionnaire involves the assessment of vividness of mental imagery in seven different sensory modalities. Tendency towards hallucinations was assessed with another standard questionnaire, the Launay-Slade Hallucination Scale (LSHS). We combined the information of self-administered questionnaires with performance measures of standardized objective psychometric tests which were assumed to share cognitive characteristics with mental imagery (Kosslyn et al., 1994). Moreover, by statistically controlling for potentially confounding factors such as general intelligence, psychomotor speed and individual psychopathology, we aimed to elucidate further the imagery abilities in schizophrenia.

\section{Methods}

\section{Subjects}

For the patient group we recruited fifty in- and outpatients of our department ( 31 males and 19 females; mean age 36.4 years; S.D. $=9.8$; range $=19-57$ ) diagnosed with paranoid schizophrenia (ICD 10: F20.0) for at least 5 years. All patients received antipsychotic medication (20 treated with atypical, 13 with typical and 17 with both). In the healthy subject group, fifty age and sex- 
matched healthy controls ( 31 males and 19 females; mean age 36.4 years; S.D. = 9.7; range $=19-57$ ), were tested. All subjects were provided with a complete description of the study and gave written informed consent before participation. Control subjects were compensated for participation.

\section{Procedure and psychometric measurements}

A test battery consisting of two questionnaires and five standardized cognitive tests (see Table 4.1 for examples and overview) were assessed in both groups. The assessment was done for every subject individually and lasted approximately one hour. The study programme furthermore included clinical ratings on the Scale for the Assessment of Positive Symptoms (SAPS) and the Scale for the Assessment of Negative Symptoms (SANS) but only 31 of the patients were willing to participate in this.

\section{Self-administered questionnaires}

The predisposition toward hallucinations was measured with the LSHS (Launay \& Slade, 1981). The LSHS consists of 12 descriptions of hallucinatory experiences. Some of the items are related to daydreams ('in my daydreams $I$ can hear the sound of a tune almost as clearly as if I were actually listening to it"), while others refer to psychotic experiences ('I have heard the voice of the devil ${ }^{\prime}$ ). For each of the 12 statements, the subjects have to score along a 5-point Likert scale (Bentall \& Slade 1985b: $0=$ certainly does not apply [to me]; $4=$ certainly applies). A high score on the LSHS indicates an increased predisposition to hallucinations. Vividness of mental imagery was assessed using the short version of Bett's Questionnaire upon mental imagery (QMI; Sheehan, 1967). The 35 items of the QMI represent statements regarding the imagery ability in seven different sensory modalities (visual, auditory, olfactory, cutaneous, kinaesthetic, gustatory, and organic). Subjects are asked to rate their imagery vividness on a 7 -point scale $(1=$ as perfectly clear as true; $7=$ I think about it, but I cannot imagine it). A low score on the QMI indicates more vivid imagery (Table 5.1).

\section{Objective psychometric test battery}

As an objective measure of different perceptual and cognitive skills, three subscales of the "General Performance Test" (Leistungsprüfsystem: LPS; Horn 1962) were assessed. The LPS represents a standardized, valid and highly reliable (total retest reliability $r_{t}=0.95$ ) measurement of fourteen different cognitive skills, which are related to Thurstone's primary mental abilities (Thurstone, 1938). We assessed the LPS subscale 10 (flexibility of closure; $r_{i 1}=$ 0.69 ), 11 (object-based speed of closure; $r_{i t}=0.71$ ) and 12 (verball-based speed of closure; $\mathrm{r}_{\mathrm{tt}}=0.88 \mathrm{j}$. Subscale 10 consists of 40 complex geometrical forms. One of five predefined target figures is embedded and thus "hidden" within these forms. The respective target figure has to be detected. Performance is subdued 
Enhanced imagery vividness and hallucinations

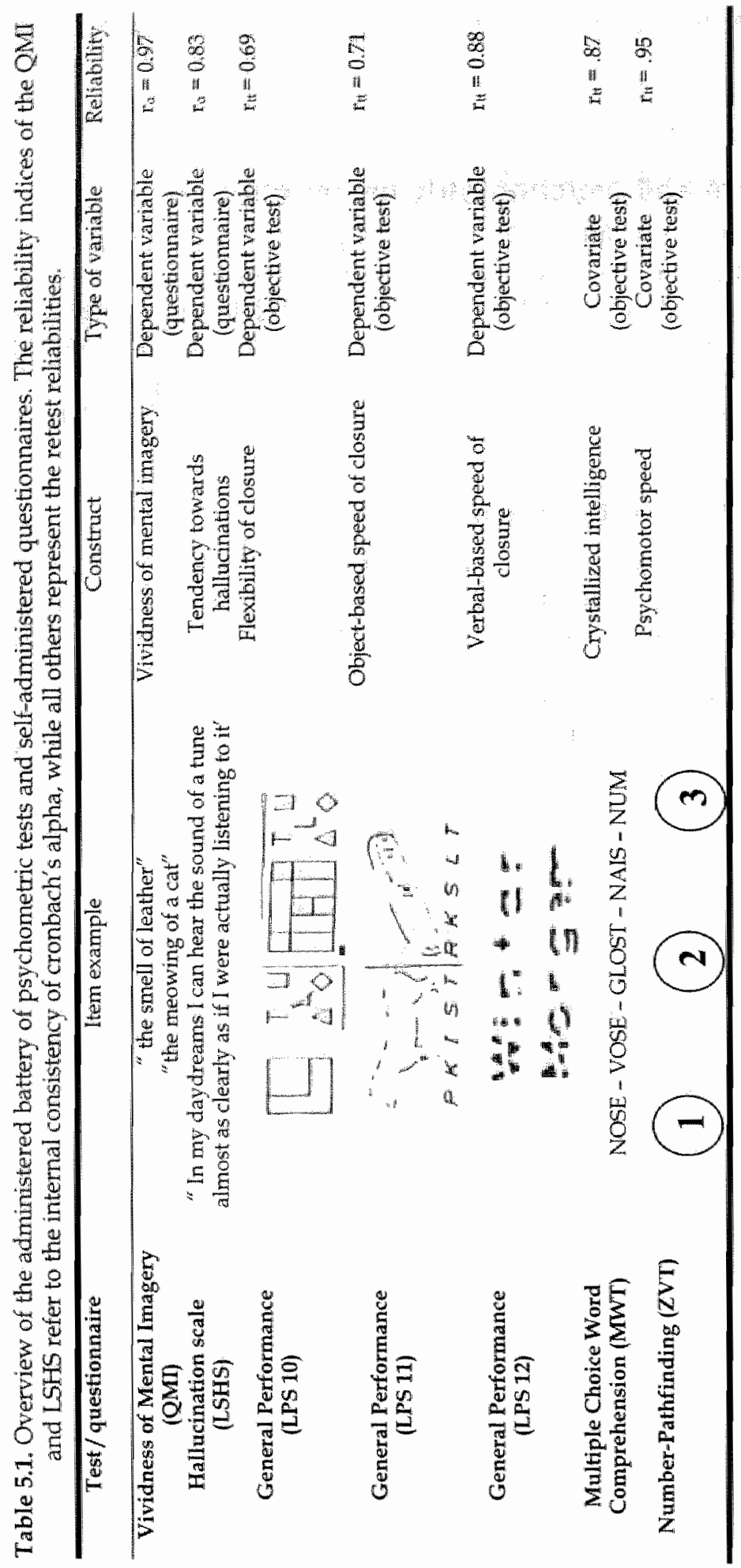




\section{Chapter 5}

to a time constraint of $3 \mathrm{~min}$. Subscale 11 requires fast object recognition. Subjects are asked to recognize 40 sketches of common objects (e.g. car apple, house), in which some pictorial parts are erased. Subjects have to recognize and name the respective objects. Subscale 12 demands a fast recognition of 40 visually degraded words. Each word contains one false letter, which has to be identified and crossed out. Performance for each of the last two tests is subdued to a time constraint of $1 \mathrm{~min}$ (Table 5.1).

\section{Psychometric covariates}

Measures of the verbal intelligence (Multiple Choice Word Comprehension test [Mehrfachwahl-Wortschatztest: MWT]; $\mathrm{r}_{\mathrm{Ht}}=.87$; Lehrl, 1989; Brüne, 2003) and of psychomotor speed (Number-Pathfinding test [Zahlenverbindungstest: ZVT]; $\mathbf{r}_{i t}=.95 ;$ Oswald \& Roth, 1987 ) were included in the test battery.

The MWT represents a widely used German test for the assessment of crystallized intelligence, which represents the semantic knowledge- and language-related factor of intelligence (Horn \& Cattell, 1966). The average correlation coefficient between the MWT (median from 32 investigations) and other global intelligence tests is relatively high at $r=0.72$, which makes the MWT a good screening instrument for general intelligence. The MWT can be considered as the German equivalent to the Spot-the-Word-Test (Baddeley et $\mathrm{al}_{.,}$1993).

The ZVT is a short, non-verbal test for examining cognitive speed. The task material consists of four numerical matrices, each comprising numerals from 1 to 90 in changing arrangements. Subjects were asked to connect these numerals in ascending order by drawing lines as quickly as possible. The mean speed in seconds for each matrix is calculated as the score for psychomotor speed. Retest-reliability for the individual administration is $r_{t t}=0.95$, the internal consistency ranges from $\alpha=0.95$ to $\alpha=0.97$ (Table 5.1). The ZVT is the equivalent to the Trail Making Test A (Reitan, 1956).

\section{Individual psychopathology}

In order to account for differences in the psychopathological symptoms of the schizophrenic patients, a subsample of thirty-one patients were interviewed and rated on the basis of the SAPS (Andreasen, 1984) and SANS (Andreasen, 1981).

\section{Evaluating the psychometric properties of the German versions of QMI and LSHS}

Prior to the psychometric assessment, the QMI and LSHS were translated into German by three independent translators. These versions were administered to a sample of 100 healthy controls in order to analyze the respective psychometric properties of the German version of the QMI and the LSHS. 


\section{Statistical analyses}

\section{Psychometric properties}

The psychometric properties of both questionnaires were analyzed. In addition, the factor structure of the QMI was assessed.

\section{Multivariate correlation analyses}

We computed multivariate correlation analyses between all tests and questionnaires of the test battery independently for both groups.

\section{Statistical group comparisons and Analyses of Covariance}

The performances of patients and controls in the different questionnaires and tests were compared and group differences were tested for statistical significance using Analysis of Variance (ANOVA). In order to statistically control for potential differences in crystallized intelligence and psychomotor speed between both groups, two Analyses of Covariance (ANCOVAs) were computed, comparing the differences in both questionnaires and the three subscales of the LPS with general crystallized intelligence and psychomotor speed as covariates.

\section{Analysis of individual psychopathology and medication}

Within the subsample $(\mathrm{N}=31)$ of patients that was assessed by standardized clinical interviews a potential effect of positive and negative symptoms on the questionnaires was investigated with a correlation analysis. In addition, a oneway ANOVA with medication as fixed factor was used to investigate the influence of different antipsychotics upon the scores on the different questionnaires.

\section{Results}

\section{Psychometric properties}

The psychometric properties of the German translation of the QMI were analyzed on the basis of 100 healthy controls. The computed exploratory factor analysis (principal components analysis, extraction criterion: eigenvalues $>1$, rotated solution) revealed seven factors that corresponded to the seven sensory modalities of imagery described by the items. The total reliability of the German QMI version proved to be very high with a cronbach's alpha of $\alpha=0.97$. The total reliability of the German LSHS (cronbach's alpha $=0.83$ ) was also satisfactory. 
Chapter 5

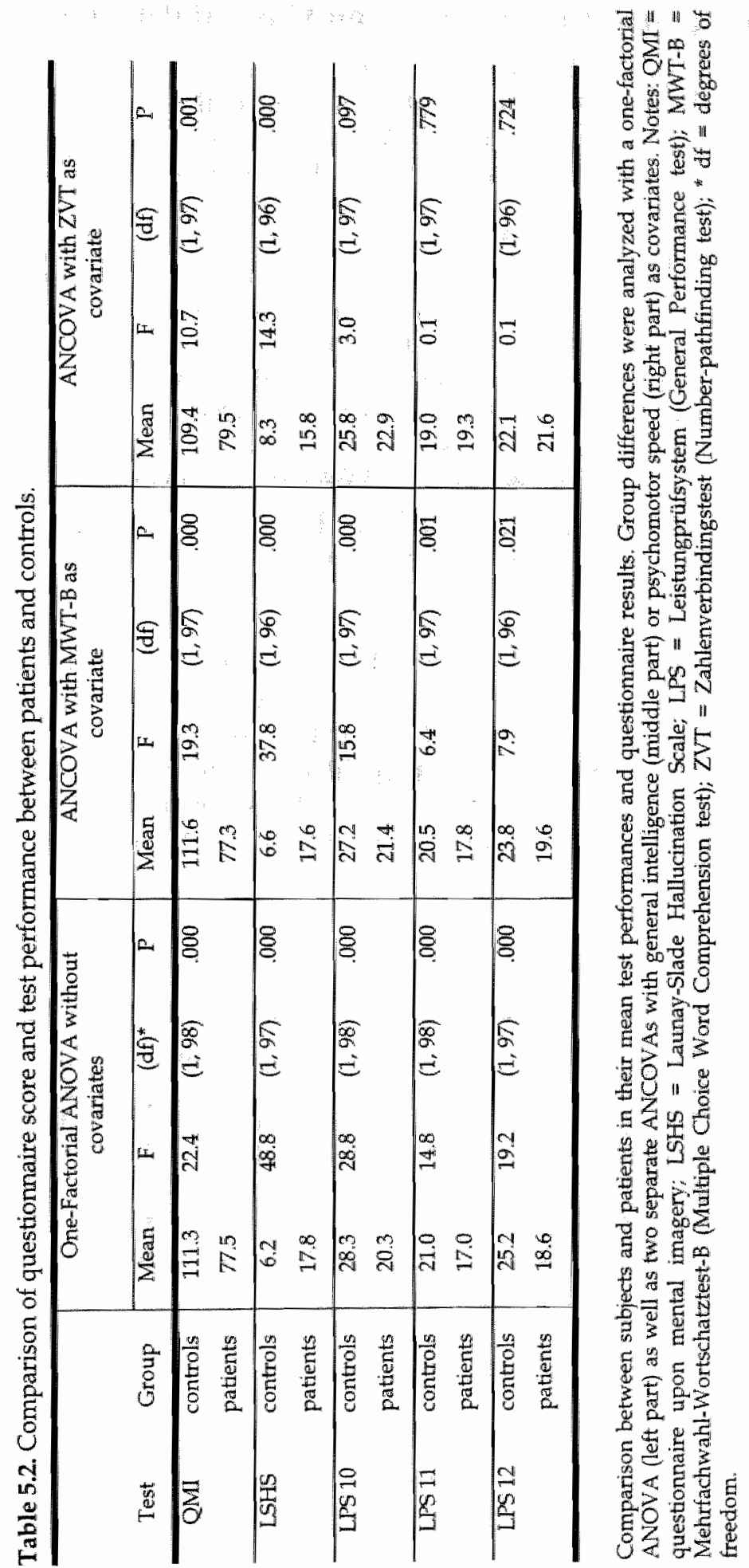




\section{Multivariate correlation analyses and factorial structures}

The principal components analysis (extraction criterion: eigenvalue $>1$; rotated solution) identified a five factor solution for the patient group's LSHS that accounted for $76 \%$ of the total variance. Within this factorial structure we were able to identify one factor (explaining $25 \%$ of the variance), which best reflected the hallucinatory experiences of the patients. For the control group the computed principal components analysis of the LSHS revealed a three factor solution which accounted for $82 \%$ of the total variance. The first factor alone accounted for $52 \%$ of the variance and could be characterized as the general hallucinatory tendency with a similar loading pattern as found for the patient group. For the following statistical analyses regarding the correlation between hallucination tendency and vividness of mental imagery we used both, the total score in the LSHS (LSHS-T) as well as the factor value representing the "cleaned" hallucinatory tendency ratings (LSHS-H) of both groups.

The exploratory factor analysis of the QMI (extraction criterion: eigenvalue $>1$; rotated solution) revealed that for the patient group, like for the sample of 100 healthy controls, a seven factor solution could be found. The loading pattern of this factorial structure clearly corresponded to the seven different sensory modalities of imagery represented by the items of the QMI.

The correlation analyses revealed no correlation between QMI and LSHS-T in either of the two groups (patients: $r=-0.205 ; \mathrm{p}=0.157$; controls: $\mathbf{r}=-0.100 ; \mathrm{p}$ $=0.491$ ), nor between QMI and LSHS-H (patients: $r=-0.108 ; p=0.556$; controls: $r=-0.005 ; p=0.980$ ). The correlation between the LSHS-T or LSHS-H and the seven subscales of the QMI representing the different sensory modality of mental imagery was not significant either.
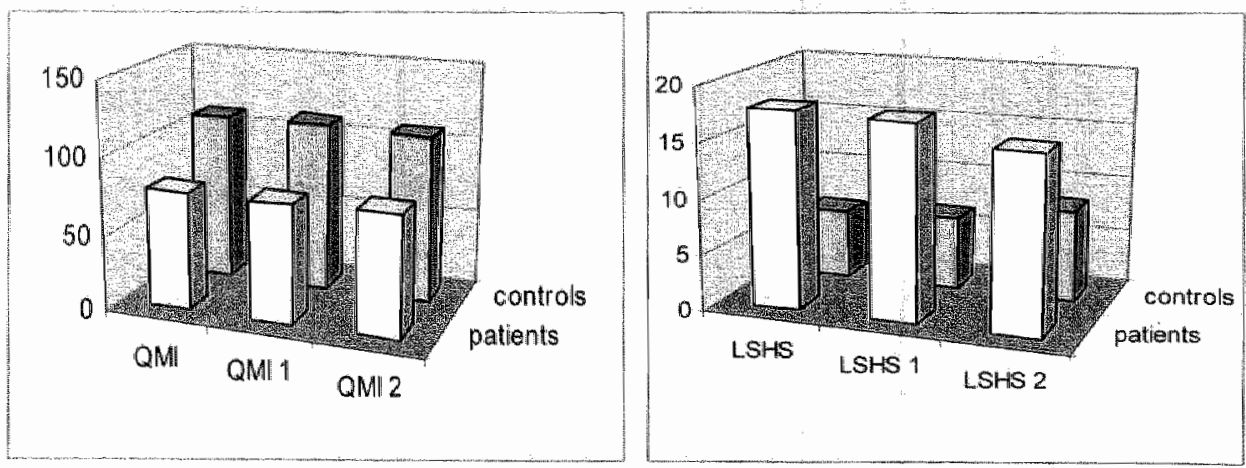

Figure 5.1. Comparison of imagery and hallucination scores for patients and controls. Mean scores on the QMI (left histogram) and LSHS (right histogram) for patients and controls as computed from the raw data (QMI. LSHS), as well as the means adjusted for crystallized intelligence (QMI 1, LSHS 1) and adjusted for psychomotor speed (QMI 2, LSHS 2). 


\section{Chapter 5}

Furthermore, no correlations could be found between the QMI and the three objective measures of perceptual closure, including flexibility of closure (patients: $\mathrm{r}=-0.085 ; \mathrm{p}=0.558$; controls: $\mathrm{x}=0.124 ; \mathrm{p}=0.391$ ), object-based speed of closure (patients: $r=-0.242 ; p=0.090$; controls: $r=0.261 ; p=0.067$ ) and verbal-based speed of closure (patients: $\mathrm{x}=-0.033 ; \mathrm{p}=0.821$; controls: $\mathrm{r}=-0.019$; $\mathrm{p}=0.896)$.

\section{Statistical group comparisons and Analyses of Covariance}

The calculated mean of the QMI was significantly lower in the patient group (patients $=77.54$ : controls $=111.30$ ). Since a lower score on the QMI indicates a more vivid imagery (see methods), the patients scored significantly higher on the imagery questionnaire $\left(\mathrm{F}_{(1,98)}=22.36: \mathrm{p}<0.001\right)$ in comparison to the controls (Figure 5.1). The separate analyses of the seven subscales of the QMI revealed that this difference in vividness of mental imagery between patients and controls was also significant when each modality of the QMI was considered separately. Hence, the schizophrenic patients showed a significantly higher vividness of mental imagery in all seven sensory modalities in comparison to the control group.

Conversely, the patients performed significantly worse in all three subscales of the LPS including flexibility of closure $\left(\mathrm{F}_{(1,98)}=28.78: \mathrm{p}<0.001\right)$, object-based speed of closure $\left(\mathrm{F}_{(1,98)}=14.83: \mathrm{p}<0.001\right)$ and verbal-based speed of closure $\left(\mathrm{F}_{(1,97)}=19.24: \mathrm{p}<0.001\right)$, while scoring significantly higher on the hallucination scale $\left(\mathrm{F}_{(1,97)}=48.81: \mathrm{p}<0.001\right)$ in comparison to the controls (Table 5.2, ANOVA).

In order to control for possible confounding effects of the differences between both groups in general crystallized intelligence and psychomotor speed on these results, two independent ANCOVAs were computed, using MWT and ZVT as statistical covariates. These analyses revealed that even after statistically controlling for the variance that can be explained by the group differences in general crystallized intelligence, the observed differences between both groups in fluid intelligence (LPS scales), vividness of mental imagery (QMI; Figure 5.1, left histogram) and tendency towards hallucinations (LSHS; Figure 5.1, right histogram) remained significant (Table 5.2, middle part).

The statistical adjustment for the influence of psychomotor speed on the group differences resulted in a non-significant difference between both groups in the three measures of fluid intelligence (LPS 10,11, 12), but left the significant differences between both groups in the QMI and the LSHS unchanged (Table 5.2 , right part).

\section{Analyses of individual psychopathology and medication}

The correlation analyses for the 31 patients who were rated with the SAPS and SANS revealed no correlation between the vividness of mental imagery or 
hallucinations (LSHS-T and LSHS-H), and positive or negative symptoms as measured by the total score in the SAPS and SANS (Figure 4.2). Additionally, we computed three different subsets of psychotic symptoms from the SAPS ratings, representing hallucinations (item 7), delusions (item 20), and passivity (item 15). The correlation analyses with these three items of the SAPS again revealed no correlation with the vividness of mental imagery (SAPS hallucinations: $\mathrm{r}=136, \mathrm{p}=.480$; SAPS delusions: $\mathrm{r}=-.066, \mathrm{p}=.733$; SAPS passivity: $\mathrm{r}=056, \mathrm{p}=.772$ ) or the tendency towards hallucinations as measured with the LSHS-H (SAPS hallucinations: $r=360, p=.130$; SAPS delusions: $r=-$ $.148, p=.454$; SAPS passivity: $\mathrm{r}=-.023, \mathrm{p}=.925$ ).

A statistical comparison of this subsample with 31 age- and sex-matched controls confirmed our result of a higher vividness of mental imagery in schizophrenic patients. Hence, there was no confounding effect of the differences in psychopathology, which is illustrated by the lack of direction within the scatterplots of Figure 4.2. The comparison between the different types of medication (three levels: typical, atypical, both) within a one-factorial ANOVA revealed no significant differences between these medication groups in the questionnaires.

\section{Discussion}

In this study we were able to show that patients with paranoid schizophrenia report more vivid mental imagery in comparison to age- and sex-matched healthy controls. This difference in vividness of mental imagery between patients and controls was also significant when each modality of the QMI was considered separately. Furthermore, the higher imagery vividness of the patients was revealed to be statistically independent of the differences in general intelligence and psychomotor speed and unrelated to the individual psychopathology of the patients, including the presence of hallucinations.

The exploratory factor analysis revealed that the factorial structure of the QMI was unchanged in the patient group, resulting in a seven factor solution corresponding to the seven modalities targeted by the questionnaire (Sheehan, 1967). The patients thus not only showed a higher vividness of mental imagery, but maintained a differentiation of this imagery ability.

The exploratory factor analysis of the LSHS revealed a five factor solution for the patient and a three factor solution for the control group. These results conform to former studies on the factorial structure of the LSHS. The loading pattern of the factor solution in the patient group was similar to the results of Levitan et al. (1996), while the loading pattern of the three factor solution in the control group largely replicated the findings by Aleman et al. (2001). 


\section{Chapter 5}
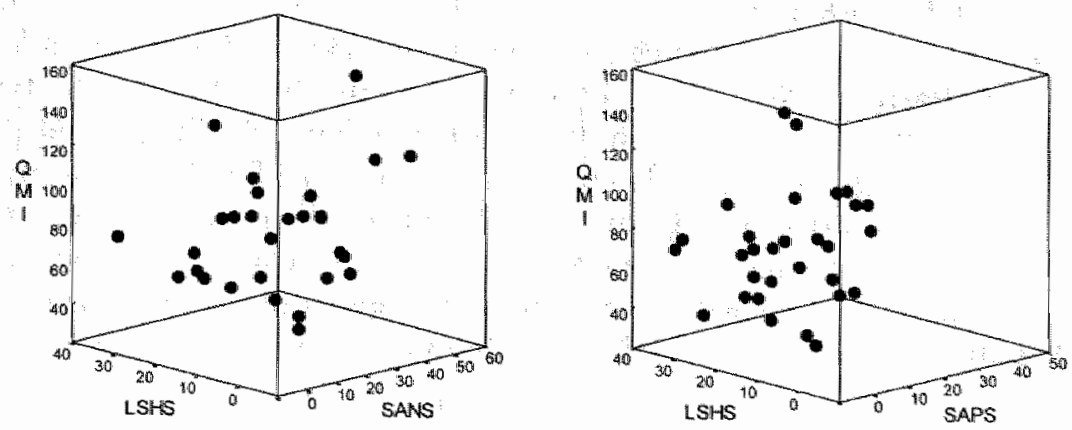

Figure 5.2. Correlation between imagery and hallucination scores and individual psychopathology. Scatterplots showing the correlations between scores on the QMI, LSHS and negative (left plot) and positive (right plot) symptoms. Table 5.3 shows the respective correlation matrix, including the correlations with the LSHS-H.

No significant correlation was found between mental imagery and tendency towards hallucinations in the patient group, which suggests that these two constructs might be independent. Nor does the higher imagery vividness of the patients seem to be an effect of the individual psychopathology. Especially the lack of correlation between the SAPS (and even the hallucination subscale) and the LSHS was unexpected. For an explanation we may turn to the difference of the time interval addressed by the two measures. While the LSHS probes hallucinatory experiences that occurred at some point in the past, the SAPS only covers a very restricted time period of one week. .

Our finding of a higher imagery vividness in schizophrenic patients is consistent with the results of Mintz and Alpert (1972), who also found a significantly higher vividness of mental imagery in schizophrenic patients. In addition, strong correlations between increased vividness of mental imagery and the presence of positive schizotypal traits have been reported (Van de Ven \& Merckelbach, 2003). However, the majority of studies did not find a significant difference in global mental imagery between schizophrenic patients

Table 5.3. Correlation matrix between imagery and hallucination scores and individual psychopathology.

\begin{tabular}{ccccc}
\hline & LSHS-T & LSHS-H & SANS & SAPS \\
\hline QMI & -0.205 & -0.108 & +0.200 & +0.036 \\
LSHS-T & & $+0.972^{*}$ & -0.067 & +0.238 \\
LSHS-H & & & -0.093 & +0.211 \\
SANS & & & & -0.118 \\
\hline
\end{tabular}

Notes: ${ }^{*}$ significant at $p<0.001$ 


\section{Enhanced imagery vividness and hallucinations}

and controls (Brett \& Starker, 1977; Starker \& Jolin, 1982; Bocker et al., 2000). even when scores on questionnaires of mental imagery were compared (Chandiramani \& Varma, 1987). This discrepancy in the literature could be brought about by differences between the studies regarding the methods employed and extent to which possible covariates were considered. Our study revealed that when information from self-administered questionnaires is combined with that from objective cognitive tests and when confounding factors such as general intelligence and psychomotor speed are controlled for, schizophrenic patients do show a much higher vividness of mental imagery.

Even so, our patient group performed worse on tasks that are supposed to recruit cognitive processes related to mental imagery (the LPS subscales). This result is compatible with theories that attribute the group difference in vividness of mental imagery not to an enhancement of mental imagery abilities as such (Aleman et al, 1999; Bentall, 1990) but to altered information processing as a result of stable beliefs (Lobban et al., 2002) or a deficient source monitoring system (McGuire et al, 1996b). However, the impaired performance on the LPS was partly explained by deficits in psychomotor speed, which further complicates the interpretation of the results.

A recent study on differences in perceptual closure between schizophrenic patients and healthy controls avoided this confound (Doninger et al., 2001). In this study a different perceptual closure task was used that did not utilize time constraints. Instead, performance was assessed by the level of picture degradation that could be manipulated, and patients were found to perform significantly worse than healthy controls. Further research will thus be needed to investigate whether patients with schizophrenia are at all able to utilize their enhanced vividness of imagery for cognitive tasks that require good mental imagery abilities. It will also be worthwhile to conduct functional imaging studies of mental imagery in schizophrenia in other modalities than the auditory (Shergill et al., 2000b) in order to reveal potential neural correlates of the enhanced vividness of the perceptual experience.

It is interesting to note that the enhanced vividness of mental imagery is independent of the severity of positive and negative symptoms, which indicates that it is not merely an effect of the current psychopathological state of the patient but related to the presence of the disease as such. In this context it could be speculated that subjectively reported enhanced vividness of mental imagery might prove to be a new trait marker of schizophrenia. However, such a claim needs to be based on further replication, including appropriate family and genetic studies. 


\section{Chapter 5}

\section{Acknowledgements}

The authors are grateful to Prof. Koruad Maurer, M.D. Head of the Dept. of Psychiatry and Psychotherapy I of Frankfurt University, and Barbara Schneider, M.D. Consultant Psychiatrist, for help with recruiting patients. Special thanks are due to Bemadette Jansma, Ph.D. Robert Bitther and Christian Roeder, M.D. provided important comments on the manuscript. Financial support by the Medical Faculty of the Johann Wolfgang Goethe University (research grant to D.L.) and the Max Planck Society ("Tandemprojekt" grant to D.L.) is gratefully acknowledged. 
Chapter 6

\section{Crystallized intelligence, perceptual abilities and symptoms of schizophrenia: An exploratory approach}




\section{Abstract}

Schizophrenia patients generally perform worse than healthy controls on many neuropsychological tests. It has been argued that test performance in schizophrenia is driven by a single fundamental neurocognitive impairment. We reanalysed performance scores of schizophrenia and control subjects on cognitive ability tests for perceptual closure abilities, crystallized intelligence, and psychomotor speed. In addition, we acquired individual psychopathology ratings via standardized interviews. Data were analysed for group differences, as well as patterns of correlations between tests for each group. Schizophrenia patients performed worse on all the cognitive ability tests. In addition, correlations between test performances within the schizophrenia group revealed a strong association between the various tests, including crystallized intelligence, in comparison to healthy controls. This finding was illustrated using exploratory principal component analyses, which yielded a single component for the patient group, and two components for the control group. However, psychopathology ratings showed a moderate dissociation, where negative symptoms correlated more strongly with perceptual ability test scores, and positive symptoms with crystallized intelligence. Our findings seem to favour the notion of a generalized cognitive deficit in schizophrenia, where negative symptoms are more strongly associated with impairments than positive symptoms. The implications of these findings are discussed. 


\section{Introduction}

Schizophrenia is a mental disorder that is characterized by a substantial heterogeneity in symptoms and behaviours. The current diagnostic manuals of clinical psychiatry acknowledge this heterogeneity by referring to a group of schizophrenic syndromes, each with different core symptoms. Besides the differences between patients, there is also a considerable intra-individual variability, with more cognitive basic symptoms in the early (pre-admission) phase of the disorder positive symptoms around the time of the first admission, and pronounced negative symptoms in a residual phase (An der Heiden \& Häfner, 2000). This heterogeneity poses a potential problem for classification of the disorder, and has motivated alternative models that emphasize underlying biological or psychological commonalities. Bleuler (1950), for example, referred to the disorder as a "group of schizophrenias" with a common cognitive pathological factor. It is generally assumed that such a factor, if present, could not be described in terms of a structural brain lesion or otherwise localized neurobiological impairment. Rather, it has been proposed that a disruption of anatomical or functional connectivity would result in the impairment of a core cognitive process (Friston, 1998; Andreasen, 1999), which would then manifest itself in a wide range of clinical symptoms. In schizophrenia, disruptions of connectivity may indeed be present at synaptic (Lewis \& Levitt, 2002) or white matter levels (Hubl et al., 2004). These attempts at unifying the variable clinical presentations of schizophrenia at the phenomenological or biological level are complemented by the search for endophenotypes (Gottesman \& Gould, 2003), many of which are determined at an intermediate level of analysis between the clinical presentation and the pathological mechanisms.

A number of studies have investigated whether a common factor contributed to performance on batteries of neuropsychological tests in schizophrenia. It is commonly observed that overall performance is worse in schizophrenia patients than healthy controls (Allen et al., 1998; Heinrich \& Zakzanis, 1998). However, further analyses of the scores of schizophrenia patients on individual subtests revealed that impaired performance could not be fully attributed to a set of specific and mutually independent cognitive deficits (Dickinson et al., 2004; Mohamed et al., 1999; Weickert et al., 2000). In a group of first-episode schizophrenia patients, the largest impairments were found on tests that tap into executive skills and that rely heavily on cognitive processing speed. However, , this core deficit resulted in impaired performance on a wide range of cognitive tests (Binder et al., 1998; Mohamed et al., 1999). Because many of these patients were neuroleptic naïve, these cognitive impairments could be attributed to the disorder independently of medication, institutionalization and chronic effects of the disorder. 
Although these results indicated that a core cognitive deficit may underlie impaired cognitive performance of schizophrenia patients, only few studies have attempted to explain the variation of test scores using a model that explicitly assumed a common factor. Using a multivariate analysis approach, Dickinson et al. (2004) examined whether a statistical model that contained a common factor, in addition to a diagnostic factor, could explain the variation between the test scores of schizophrenia patients and healthy controls. These authors found that, for the schizophrenia group, the common factor alone explained about two-thirds of the difference in test scores. This finding indicated that for patients, but not healthy participants, the general impairments across a wide variety of cognitive tests could be driven by a single pathological factor. However, the nature of this factor remains to be elucidated.

In a previous study we found that a group of schizophrenia patients could be characterized uniquely by reports of introspectively judged increased vividness of mental imagery (Sack et al., 2005; see chapter 5 of this thesis). This finding proved to be independent of individual psychopathology, including hallucinations, and was not restricted to only the visual or auditory domain. In addition, we used a small set of cognitive ability tests to further characterize the cognitive profiles of the patient group. Here, we report on a follow-up analysis of the data. We investigated, in an exploratory fashion, whether the pattern of cognitive abilities would be different between the schizophrenia and control groups, and if so, what this meant for the relation between cognitive abilities and symptoms.

\section{Methods}

\section{Subjects}

Fifty in- and outpatients ( 31 males and 19 females; mean age 36.4 years; S.D. = 9.8; range $=19-57$, diagnosed with paranoid schizophrenia (ICD 10: F20.0; World Health Organization 1992) for at least 5 years, participated in this study. At the time of participation, all patients received antipsychotic medication (20 treated with atypical antipsychotics, 13 with typical and 17 with both classes). As a control group, fifty age- and gender-matched healthy subjects ( 31 males and 19 females; mean age 36.4 years; S.D. $=9.7$; range $=19-57$ ), were tested. All participants were provided with a complete description of the study and gave written informed consent before participation. Control subjects were compensated for participation.

\section{Materials and procedures}

In this study we reanalyzed a small test battery, which is fully described in Sack et al. (2005). Here, the design and application of the battery is briefly considered. 


\section{Chapter 6}

We assessed performance on three tests of perceptual and cognitive skills, one test of general crystallized intelligence, and one test for psychomotor speed. For the perceptual and cognitive skills, three subscales of the "General Performance Test ${ }^{\prime \prime}$ (Leistungsprüsystem: LPS) (Horn, 1962) were assessed (subscales 10, 11 and 12). The LPS represents a standardized, valid and highly reliable (total retest reliability $\mathrm{r}_{\mathrm{tt}}=0.95$ ) measurement of fourteen different cognitive skills, which are related to Thurstone's primary mental abilities (Thurstone, 1938). Subscale 10 measures flexibility of closure, and contains 40 test-items that consist of complex geometrical forms. One of five predefined target figures is embedded within the test-item, ${ }_{i}$ and has to be defected as quickly as possible. Subscale 11 measures object-based speed of closure, and contains 40 black-and-white fragmented line drawings. Participants are required to judge the identity of the object that is represented as quickly as possible. Subscale 12 measures verbal-based speed of closure, and contains 40 nouns, which are visually degraded. Every word contains one false letter, which has to be identified and crossed out. The time constraints on the performance of subscale 11 is 3 minutes, while for subscales 11 and 12 it is 1 minute.

General crystallized intelligence, which represents the semantic knowledge- and language-related factor of intelligence (Horn \& Cattell, 1966), was assessed using the Multiple-Word-Choice test ([MehrfachwahlWortschatztest: MWT]; $r_{t t}=0.87$; Lehrl, 1989). The test is independent from psychic and mental illnesses. Requirements of fluid intelligence respectively mental functions are propositionally small. The average correlation coefficient between the MWT (median from 32 investigations) and other global intelligence-tests is relatively high $(r=0.72)$. The test is comparable to the Spotthe-word-test (Baddeley et al., 1993).

Psychomotor speed was assessed using the Number-Pathfinding test ([Zahlen-verbindungs-test: ZVT]; $\mathrm{r}_{\mathrm{tt}}=.95 ;$ Oswald \& Roth, 1987). The ZVT is a short, non-verbal test for examining a person's cognitive speed. Task materials consist of four numerical matrices, each comprising numerals from 1 to 90 in differing arrangements. Subjects were asked to connect these numerals in ascending order by means of lines as quickly as possible. The mean speed for each matrix is calculated as the score. The ZVT is equivalent to the Trail-Making Test (Reitan, 1956).

For the schizophrenia patients, individual psychopathology was assessed using the Scale for the Assessment of Positive Symptoms (SAPS: Andreasen, 1984), and the Scale for the Assessment of Negative Symptoms (SANS: Andreasen, 1981). Only a subsample of 31 patients was willing to participate in these interviews.

Assessment of the tests was done for all participants individually. Data registration and statistical analyses were done using SPSS 10 for Windows. The scores of the questionnaire, cognitive ability tests and psychopathology ratings 
were standardized by subtracting the variable means and dividing the scores by the variable standard deviation ( $Z$-scored).

\section{Results}

A multivariate analysis of variance (MANOVA) of the three categories of medication (typical, atypical, both) upon the test and symptom interview scores did not reveal any significant differences (all $\mathrm{p}^{\prime} s<0.05$ ). Therefore, the factor of medication was excluded from further analyses, and the patient group was treated as one group. Initial analysis of the probability distributions of the tests

(Kolmogorov-Smimoff [K-S]) showed that the distributions for MWT and ZVT were significantly different from normal (MWT: $\mathrm{K}-\mathrm{S} \mathrm{Z}=1.91, \mathrm{p}=0.001$; $Z V T$ : $K-S Z=1.79, p=0.003$ ). Group differences were assessed using a parametric test where appropriate (T-tests), while the variables with nonnormal distributions were analyzed using the non-parametric Mann-Whitney-U test. For the parametric two-sample T-tests, Levene's test for equality of variance across groups revealed highly significant differences (all $\mathrm{p}^{\prime} \mathrm{s}<0.01$ ), where the variances for the patient group were consistently larger in comparison to the control group. Therefore, the corrected degrees of freedom were used to assess the appropriate t-values. All group differences proved to be highly significant at an alpha of 0.05 (see Table 6.1). The patient group had higher scores on the ZVT, indicating a slowed psychomotor performance, and lower scores on the LPS subscales and the MWT, indicating lower fluid and general crystallized intelligence.

Table 6.1. Means for the schizophrenia (SCZ) and control groups on the Z-scored LPS subscales, the MWT and the ZVT:.

\begin{tabular}{|c|c|c|c|c|c|c|c|c|}
\hline \multirow[b]{2}{*}{ Test } & \multirow[b]{2}{*}{ Measure } & \multicolumn{2}{|c|}{ SCZ } & \multicolumn{2}{|c|}{ Control } & \multirow[b]{2}{*}{$d f$} & \multirow[b]{2}{*}{ Stat: } & \multirow[b]{2}{*}{ P } \\
\hline & & $\begin{array}{l}\text { Mean } \\
\text { (SD) }\end{array}$ & $N$ & $\begin{array}{c}\text { Mean } \\
\text { (SD) }\end{array}$ & $\mathrm{N}$ & & & \\
\hline \multirow[t]{3}{*}{ Par } & LPS10 & $\begin{array}{r}-0.47 \\
(1.00)\end{array}$ & 50 & $\begin{array}{c}0.47 \\
(0.47)\end{array}$ & 50 & 91.03 & 5.37 & $<0.001$ \\
\hline & LPSII & $\begin{array}{r}-0.36 \\
(1.18)\end{array}$ & 50 & $\begin{array}{c}0.36 \\
(0.60)\end{array}$ & 50 & 72.35 & 3.83 & $<0.001$ \\
\hline & LPS12 & $\begin{array}{r}-0.40 \\
(1.13)\end{array}$ & 49 & $\begin{array}{c}0.40 \\
(0.65)\end{array}$ & 50 & 76.42 & 4.36 & $<0.001$ \\
\hline \multirow[t]{2}{*}{$\begin{array}{c}\mathrm{N} \\
\text { par }\end{array}$} & MWT & 39.45 & 49 & 60.34 & 50 & & 708.0 & $<0.001$ \\
\hline & $Z \mathrm{VT}$ & 70.10 & 50 & 30.90 & 50 & & 270.0 & $<0.001$ \\
\hline
\end{tabular}

For the parametric two-sample T-tests (Par), means and standard deviations (SD) of the Z-scores are given. For the non-parametric Mann-Whitney-U Test (Npar), mean ranks are presented. Notes: LSHS = Launay-Slade Hallucination Scale; LPS = Leistungsprüfsystem; MWT = MehrfachwahlWortschatz-Intelligenztest; $Z \mathrm{VT}$ = Zahlen-Verbindungs-Test; a degrees of freedom (df) are corrected for unequal variances between groups. 
Table 6.2. Correlation coefficients between the cognitive ability hests for whe schizophrevia ( $S C Z$ ) and control groups.

\begin{tabular}{|c|c|c|c|c|c|c|c|c|}
\hline \multirow[t]{2}{*}{ 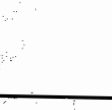 } & \multirow[b]{2}{*}{ LPS10 } & \multicolumn{2}{|c|}{$S C Z$} & \multirow[b]{2}{*}{ MWT } & \multirow[b]{2}{*}{ LPS10 } & \multicolumn{2}{|c|}{ Controls } & \multirow[b]{2}{*}{ MWT } \\
\hline & & LPS11 & LPS12 & & & LPSII & LPS12 & \\
\hline LPSII & 0.73 & & & & 0.54 & & & \\
\hline LPSI2 & $0.78^{c}$ & 0.67 & & & 0.54 & 0.25 & & \\
\hline $\mathrm{MWT}$ & 0.57 & $0.35^{\mathrm{a}}$ & $0.60^{\circ}$ & & 0.02 & 0.08 & 0.21 & \\
\hline ZVT & -0.55 * & $-0.56^{k_{\mathrm{e}}}$ & $-0.61^{k_{2}}$ & $-0.33^{*_{\mathrm{a}}}$ & -0.32 & -0.24 & $-0.32 \pi$ & -0.1 \\
\hline
\end{tabular}

Correlation coefficients are Pearson product-moment correlations, except where denoted $\left.{ }^{*}\right)$. Notes: a $\mathrm{p}<0.05 ; \mathrm{c} p<0.001$.

One-sample K-S tests on the probability that the underlying distributions approached normality were done for the cognitive tests (LPS 10, 11, 12, MWT and ZVT) for both groups separately, as well as the psychopathology ratings (SAPS and SANS) for the patient group. For the control groups, none of the distributions deviated significantly from normality $(\alpha=0.05)$. For the patient group, the distributions of the ZVT and the SAPS differed significantly from normal (ZVT: $K-S Z=1.6, p=0.011$; SAPS: $K-S Z=1.5, p=0.022$ ). All correlation tests that involved these variables were performed using nonparametrical versions (Spearman rank), while in the other cases parametric tests were used (Pearson's product-moment).

Table 6.2 lists the correlations for the tests of cognitive abilities for the patient and the control group. Correlations between the LPS subscales were stronger in the patient group. While in the control group subscales 11 and 12 were not significantly correlated, all subscales of the patient group showed very high correlations $(\mathrm{r}>0.6)$, with p-values smaller than 0.001 . At the same time, the LPS subscales correlated very strongly and positively with general crystallized intelligence, and negatively with psychomotor speed. These patterns were much less strong, or even absent in the control group.

Exploratory principal component analyses (PCAs) of the scores of the schizophrenia group and the control group corroborated our findings. With an extraction criterion of eigenvalue $>1$, PCA for the patient group revealed only 1 principal component, while PCA for the control group revealed 2 components. The single factor for the patient group represented both flexible cognitive abilities and semantic knowledge, and explained $66.13 \%$ of the total variance. The first factor for the controls represented flexible cognitive abilities (explained variance $=43.13 \%$ ), and the second factor represented semantic knowledge (explained variance $=20.51 \%$ ) (Table 6.3).

Correlations between individual psychopathology and the cognitive ability tests indicated that impaired performance on these tests was primarily associated with negative symptoms (see Table 6.4). More specifically, performance on flexibility and object-based speed of closure were negatively correlated with severity of negative symptoms, while worse performance on 
psychomotor speed (i.e., a lengthening of performance time) was positively correlated to the severity of negative symptoms. Severity of positive symptoms, as assessed by SAPS, showed a negative correlation with performance on the crystallized intelligence scale, as well as on object-based speed of closure. The pattern of correlations thus provides some indication of a moderate dissociation of the relationships of symptomatology and impairment in certain cognitive domains.

To evaluate the contribution of psychopathology ratings to the first principal component of the test scores, we performed a multiple regression analysis with the SAPS and SANS scores as regressors uon the principal component. At a significance level of $a=0.05$, SANS proved to be highly significant $(F(1,28)=8.65, p<0.01)$, while SAPS showed a weak trend $(F(1,28)=$ $3.21, \mathrm{p}=0.085$ ). Figure 6.1 shows the scatter plots for these variables. The explained variance of the whole model was moderate $\left(R^{2}\right.$ adjusted $=0.24$; $\mathrm{F}(1,28)=5.39, \mathrm{p}=0.011)$, indicating that symptom classification and severity leave a substantial proportion of the variation within the principal component unexplained.

Table 6.3. Factor loadings of the cognitive ability tests unto the principal components (unrotated) of the schizophrenia (SCZ) and the control group.

\begin{tabular}{ccccc}
\hline & SCZ & \multicolumn{2}{c}{ Controls } \\
\cline { 2 - 4 } \cline { 3 - 4 } & 1 & 1 & 2 \\
\hline LPS 10 & 0.904 & 0.837 & -0.269 \\
LPS 11 & 0.834 & 0.693 & -0.279 \\
LPS 12 & 0.913 & 0.747 & 0.194 \\
MWT & 0.652 & 0.246 & 0.911 \\
ZVT & -0.732 & -0.568 & -0.082 \\
\hline
\end{tabular}

\section{Discussion}

In this study, we reanalyzed a dataset that consisted of scores on a test battery of cognitive abilities and individual psychopathology in a group of schizophrenia patients and a healthy control group (Sack et al., 2005). The battery of cognitive ability tests included three tests for perceptual and cognitive abilities, one test for general crystallized intelligence, and one psychomotor speed test. We focused on the pattern of relations between these measures across the two groups. Patients performed worse on all tests and revealed a larger variability of performance scores, in comparison to healthy controls. The correlation matrix of the patient group revealed increased and more pronounced correlations between all cognitive ability tests than in the healthy control group. More specifically, the perceptual and cognitive abilities 


\section{Chapter 6}

showed a strong correlation amongst the three subscales, and with the verbal task that probed general crystallized intelligence. These findings were further supported by PCA, which revealed a single component for the patient group and two components for the control group. The individual psychopathology scores of the patient group revealed a dissociation of the links between symptomatology and the cognitive ability tests. While severity of negative symptoms was rellated to impaired performance on perceptual closure abilities and psychomotor speed, severity of positive symptoms, including haliucinations, was related to impaired performance on crystallized intelligence and object-based speed of closure. The regression of symptom severity upon the principal component of the test scores showed that negative symptoms explained most of the variance, while positive symptoms did so only weakly, suggesting that this dissociation may be moderate.

Schizophrenia patients commonly have shown impaired performance on a wide variety of neuropsychological tests, with the least impairment in semantic knowledge and the largest effects on tests that measure executive skills and cognitive speed (Allen et al., 1998; Heinrich \& Zakzanis, 1998; Mohamed et al., 1999; Weickert et al., 2000). The pattern of correlations in the control group, where performance of perceptual closure abilities and semantic knowledge are separated, is in accordance with the well established differentiation of cognitive abilities into fluid and crystallized intelligence (Horn \& Cattell, 1966). However, the increased coherence of scores on these measures within the patient group can be better described by a general intelligence factor sensu Spearman (1923).

Our finding is in support of a study by Dickinson et al. (2004), who investigated the performance of schizophrenia patients on a large intelligence test battery using structural equation modeling. In their analysis, the overall differences of test scores between the schizophrenia and healthy control groups were divided into a common factor that accounted for covarying differences between groups, and test-independent differences, which are the test differences across groups that did not covary with group differences on other tests. The common factor explained two-thirds of the variance within the schizophrenia group, suggesting that test score patterns of schizophrenia patients were different from healthy controls in that correlation between subtests was considerably higher in the patient group. In our study, we also found that the single principal factor solution for the test scores of the schizophrenia group explained about two-thirds of the variance. In light of these findings, the neuropsychological profile of schizophrenia may be more accurately described through a generalized impairment, rather than through a number of isolated cognitive deficits.

Our study also revealed a moderate dissociation of positive and negative symptoms with test scores, which may contradict the notion of a generalized deficit in schizophrenia. The negative correlation between severity of negative symptoms and the different measures of perceptual closure indicates that patients with less severe negative symptoms performed better on these tasks. 
This finding is consistent with the results of Doniger et al. (2001), who also reported worse performance on a perceptual closure task with increased severity of negative symptoms. Furthermore, in our study, severity of negative symptoms also correlated with speed of completion of the ZVT, but not with crystallized intelligence (MWT). The ZVT measures speed of cognitive processing (Oswald \& Roth, 1987), and has been found to explain a large part of the performance on perceptual closure tasks (Sack et al., 2005). Several other studies have found correlations between negative symptoms and completion time on a psychomotor speed task (Berman et all, 1997; Jogems-Kosterman et al., 2001), where the effect was attributed to impairment of a cognitive, rather than a motor component. Severity of negative symptoms may impair performance of tests that rely more on cognitive flexibility than on more hardwired cognitive abilities like semantic memory. Our pattern of correlations is in support of this notion.
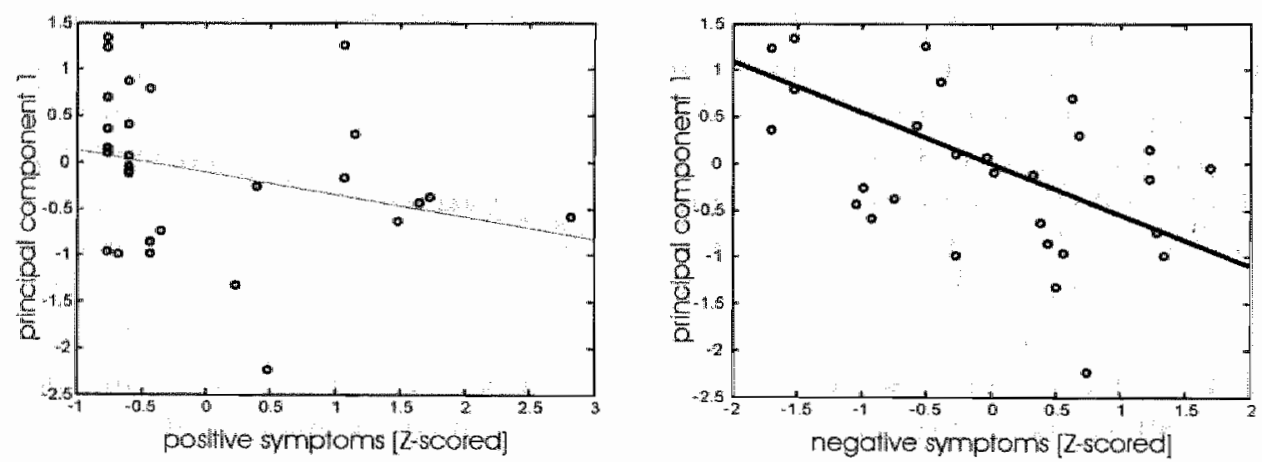

Figure 6.1. Scatter plots for the positive (left) and negative symptoms (right) vs, the first principal component for the schizophrenia patients. Thick line indicates significance $(\mathrm{p}<0.05$ ). Notice the non-normal distribution of the positive symptom scores.

Table 6.4. Correlation coefficients between psychopathology ratings and the cognitive ability tests (Npatients $=30$ ).

\begin{tabular}{|c|c|c|}
\hline & SAPS & SANS \\
\hline SANS & $-0.01^{*}$ & \\
\hline LPSIO & $-0.32^{k}$ & $-0.57^{b}$ \\
\hline LPSI1 & $-0.38^{w_{1}}$ & $-0.40^{a x}$ \\
\hline LPSI2 & $-0.28^{*}$ & -0.26 \\
\hline MWT & $-0.43^{\text {* } \text { in }}$ & -0.04 \\
\hline $\mathrm{ZVT}$ & $0.07^{*}$ & $0.43^{\text {*at }}$ \\
\hline
\end{tabular}

Correlation coefficients are Pearson product-moment correlations, except where denoted $\left({ }^{\star}\right)$. Notes: " $p<0.05 ;, p<0.01 ;$ SAPS = Scale for assessment of positive symptoms; SANS = scale for assessment of negative symptoms. 


\section{Chapter 6}

Severity of positive symptoms correlated most strongly with semantic knowledge, but also with object-based speed of closure. Both auditory verbal hallucinations and formal thought disorder have been related to impairments of language components, but with different contributions. While auditory hallucinations have been related to word production and perception problems (Kerns et al., 1999; Stephane et al., 2001), formal thought disorder may be more related to impairments of semantic memory (Aloia et al., 1998). Thus, auditory hallucinations may have a larger impact on more flexible, cognitively demanding aspects, while thought disorder may affect to a larger extent semantic knowledge. To verify whether our data reflected these findings in the literature, a post-hoc multivariate regression analysis was performed on the tests for object-based speed of closure (LPS 11) and semantic knowledge (MWT), with the summed scores of the hallucination and thought disorder subscales of the SAPS as regressors. Severity of thought disorder was significantly related to impairments of semantic knowledge $(\mathrm{F}[1,28]=7.6, \mathrm{p}=$ 0.01 , while hallucinations showed a moderate trend for object-based speed of closure $(F[1,28]=3.0, p=0.097)$. These results thus support an association between thought disorder and impaired semantic knowledge in schizophrenia, while leaving the role of hallucinations unanswered.

What do we know about the nature of the putative common pathological factor, as represented by the principal component solution? It is possible that the increased correlation between the test scores was driven by medication. However, a generalized cognitive deficit was also found in a large sample of neuroleptic naîve patients (Mohamed et al., 1999), making medication or institutionalization unlikely candidates. In line with their findings is that class of medication did not significantly explain the variation of test scores in our study. Alternatively, it may be the case that the cognitive impairments are better interpreted in terms of a bifurcation of symptoms, rather than a single underlying factor. But in this case, it should be noted that a substantial portion of the patients (about $25 \%$ ) of this study did not suffer from positive symptoms at all at the time of measurement. At the same time, theoretical models of disrupted connectivity as a common factor in schizophrenia allow for some variation in the constellation of symptoms and cognitive impairments (Andreasen, 1999). A cognitive candidate for the common factor could be psychomotor speed, as it covaries substantially with the scores on the perceptual ability tests (Sack et al., 2005). This would mean that test performances are impaired in schizophrenia because of a general, unspecific slowed information processing. However, the finding that the single principal component in the patient group contributed largely to semantic knowledge, which did not correlate with psychomotor speed, suggests that a cognitive factor other than psychomotor speed must play a role. A working memory dysfunction has been suggested to be this core cognitive deficit (Silver et al,, 2003). Impairments of the centrall executive as well as maintenance components were indeed found for schizophrenia patients on verbal and visual working memory tests (Kim et al., 2004), with the central executive component showing 
the largest impairment. Interestingly, working memory deficits were found to correlate primarily with the severity of negative symptoms (Kim et al., 2004; Silver et al., 2003). Further studies will thus be needed in order to determine the extent to which working memory deficits contribute to impairments of other cognitive domains, as well as to the pattern of symptoms.

In this study we did not utilize the complete intelligence scale, which constraints our inferences with respect to intelligence and schizophrenia. Replications of this study using a wider range of intelligence tests are needed to further validate our findings, while longitudinal assessments could shed light on the nature of the relationship between symptoms and cognitive impairments. To circumvent the potential confounds of medication on test performances, neuroleptic naïve patients, as well as schizophrenia-related participants could be studied. This could be extended further into non-clinical populations, in line with the hypothesis that schizophrenic symptoms exist on a continuum between non-pathological and clinical populations (Bentall, 1990).

In conclusion, using an exploratory approach, we found evidence for a general common factor that may underlie the performance of a variety of cognitive abilities in schizophrenia. In addition, individual psychopathology and cognitive performance in schizophrenia seemed to suggest a moderate dissociation, with negative symptoms being more closely associated with impaired "fluid" perceptual and cognitive abilities, and positive symptoms with impaired semantic knowledge. However, severity of negative symptoms was more strongly associated with impaired cognitive performance in schizophrenia, which indicated that the putative generalized cognitive impairment may be more associated with negative rather than positive symptoms. 


\section{Summary}

In this thesis the investigation of the neural and cognitive underpinnings of auditory verbal hallucinations (AVH) in schizophrenia is framed as a multivariate problem. The functional-anatomical substrates are investigated using functional magnetic resonance imaging ( $(\mathrm{MRI})$, which allows for the noninvasive recording of brain activity when patients experience their auditory hallucinations. The functional data is then analysed using recently developed multivariate statisticall tools, where a first attempt is made to consider the functional data independent from patients' self-reports.

\section{Hallucinations and connectivity}

Chapter 1 introduces spatial ICA (or SICA) as a tool to assess functional connectivity in fMRI data in the case where no model of brain activity is present a priori. Functional connectivity is defined as the correlation of activity between a set of spatially separate brain areas. SICA was applied to functional data obtained while healthy subjects did not engage in any predefined task, which is referred to as a resting state. This state plays an important role in functional imaging studies in general, because the resting state is often used as a baseline, or control measurement to which experimentally manipulated measurements are contrasted, thus assuming that during rest the brain shows an absence of activity. At the same time, such data are not easily analysed because there is no a priori model of brain activity. SICA is well suited for the analysis of such data because it does not require the formulation of an a priori model of brain activity, and can be applied to the whole brain at once. The study in chapter 1 shows that during rest the brain shows activity that is structured in space as well as in time. Within functionally specific sensory and motor areas, such as the visual, auditory and motor cortex, activity is coupled to a large degree in the absence of an explicit task, revealing the large contribution of low to very low frequencies. The spatio-temporal structure for these areas crosses hemispheres, where functionally and anatomically homologue areas in the two hemispheres shows a high degree of dependence. In addition, such patterns of spatio-temporal structure were also found for supramodal cortical areas in the frontal and parietal lobes that have been related to attentional and monitoring processes. These results indicate that the resting state contains a high amount of 


\section{Summary}

spatial and temporal structure, which not only has implications for the study of the "resting brain", but also should be considered when using the resting state as baseline for task activity comparison.

Chapter 2 presents a first application of sICA to functional data of AVH. In this study the pattern of activity within the primary auditory cortex during AVH is investigated. The functional data were analysed in a way that loosens the restrictions of a predefined model of brain activity that is based on patients' self-reports. After using sICA to decompose the functional data into a set of spatial components, the timecourses of the data that were related to auditory components were visualized using BOLD-image plots, which allow the experimenter to visually assess the amount of variability of single-trial limecourses. The study shows that, for both hallucinations as well as acoustic stimulation, auditory components show a high amount of variability in onset timing, amplitude and width of the hemodynamic responses. These findings indicate that, for at least some patients, auditory cortex - including primary auditory cortex - may be active during the experience of AVH.

Chapter 3 presents an extended application of sICA to AVH. The study reveals increased activity of bilateral, though somewhat right-lateralized, primary and secondary auditory cortex, which is a replication of the findings in the previous chapter. Furthermore, a network of frontotemporal areas, which included inferior frontal gyrus, superior temporal gyrus, medial frontal areas and inferior parietal cortex, was found that shows increased activation during hallucinations, and was shown to be left lateralized in all patients. Right middle temporal gyrus was represented in a single component, separately from the other components of interest. These networks do not reveal a timing difference with respect to the hemodynamic signal of the button presses.

In chapter 4 the issue of conducting and interpreting SICA decomposition in the subject space (i.e., sICA across subjects) is investigated using an approach that preserves the spatio-temporal information of single subject decompositions. The approach is to identify components of functional connectivity that may be associated with different attentional systems. In this study, subjects had to respond when they detected randomly presented, infrequent target stimuli amongst a train of frequent standard (task-irrelevant) stimuli. In addition, subjects were shown infrequent, task-irrelevant stimuli to which they did not need to respond. SICA is used to decompose the individual datasets into sets of components of connectivity. The individual results are then clustered across subjects, using a hierarchical clustering approach that the spatial correlations between the components as clustering criterion. The results show different connectivity networks that are related to 1) targets but not distractors, 2) distractors but not targets, and 3) targets and distractors across all subjects. 


\section{Hallucinations and cognition}

Chapter 5 and 6 present the analyses of a set of cognitive and perceptual tasks, and mental imagery and hallucination questionnaires that were presented to paranoid schizophrenia patients and healthy individuals. The cognitive tasks tapped into psychomotor speed and crystallized intelligence. The perceptual tasks measured aspects of perceptual closure, where subjects had to visually complete objects or written words, and had to extract geometric targets from a complex constellation of figures. Subjects were also given self-report questionnaires about the vividness of their mental imagery and the predisposition to hallucinate.

The scientific literature does not show an agreement on whether or not hallucinations are related to an increased vividness of imagined percepts. In the study described in chapter 5 the relation between the presence of hallucinations and mental imagery is assessed. A small set of perceptual and cognitive ability tests was used as covariates in order to investigate the nature of the relation between hallucinations and mental imagery. The results showed that schizophrenia patients as a group reported increased vividness of mental imagery. However, no evidence was found for a linear relationship between vividness of mental imagery and predisposition to hallucinate. Self-reported imagery vividness and predisposition to hallucinate did not depend on psychomotor speed or crystallized intelligence. In addition, individual psychopathology ratings did not correlate significantly with the mental imagery scores. These findings suggest that increased (self-reported) vividness of imagery is strongly related to schizophrenia, regardless of the degree and severity of hallucinations.

In chapter 6 the data obtained in the previous study is reanalysed using principal component analysis. More specifically, the differences in the relations between perceptual and cognitive ability test scores between groups is investigated. The perceptual tests comprised three tests of perceptual closure, the cognitive tests comprised a test for crystallized intelligence and psychomotor speed. From psychometric intelligence models it was assumed that perceptual closure scores - which can be referred to as an aspect of fluid intelligence - should be uncorrelated to scores of crystallized intelligence. For the schizophrenia group the test scores and their relations were correlated with indlividual psychopathology ratings. The results indicated that while the test scores within the healthy group reflected the crystallized vs. fluid intelligence dichotomy (two principal components), the test scores for the schizophrenia group proved to be highly correlated (one principal component). It was unlikely that the increased associations were due to medication. While some of the test scores correlated moderately with positive symptomatology, the principal component in general correlated most strongly with negative symptoms. Thus, the difference in component structure suggest that the variation in test scores within the schizophrenia group represents the presence 
of a single (cognitive) pathological factor, rather than a multitude of widespread but independent cognitive impairments. This putative factor may be largely expressed via the negative symptoms.

\section{Conclusions and future directions}

The main findings of this thesis can be summarized as follows. The application of spatial ICA to functional imaging data of hallucinations provides additional insight into the neural activity related to AVH. Primary auditory cortex may not be a general characteristic of auditory hallucinations in schizophrenia patients, but in the cases that it does, the temporal profile is not easily modelled by self-reported onsets and durations of the experience.

At the same time, increased vividness of mental imagery is not a unique characteristic for hallucinations, but extends to the (paranoid) schizophrenia group as a whole. The associated cognitive impairments may be the expression of a single pathological factor, which is phenotypically expressed largely via negative symptoms.

There are currently no available means to study hallucinations separate from the subjectivity of the patients that experience them. However, the application of data-driven analyses may offer an alleviation of the need to rely on self-reports of these aberrant experiences. It may perhaps be possible to investigate such subjective and pathological phenomena in the absence of overt self-reports, which may allow for a less confounded study of hallucinations, and simultaneously improve patient recruitment. Concurrently, such approaches could reveal new insights about the neural underpinnings of hallucinations, as well as the state- or disease-related functional processes in which such hallucinations may reside. Recent studies have applied data-driven techniques to the schizophrenic default brain state, and have revealed that auditory cortex shows a functional pattern that is different from the healthy default state.

These results suggest several lines of future directions in research. Why do not all hallucinating patients show primary sensory cortex activity? Is it possible that such activity is associated with the phenomenological content of the hallucinations? Several studies have shown that higher-order, but not primary auditory cortex, is active during auditory mental imagery in healthy controls and non-hallucinating patients. What, indeed, is the role of the primary auditory cortex in the experience of hallucinations? On a different level, it is also not well understood in what sense functional and anatomical connections between sensory cortex and higher-order areas play a role in hallucinations in schizophrenia. Combining analysis tools with methods for measuring functional and anatomical connectivity (e.g., diffusion tensor imaging) will likely be the path to take in order to answer these questions. Individual differences in psychopathology, as well as neuropsychological and psychosocial 


\section{Summary}

functioning may provide further means in order to understand the complex and highly dynamic aspects of hallucinations, specifically, and schizophrenia in general. At the same time, many of these questions require the recruitment of schizophrenia patients, especially in the context of cognitive or neural diseaserelated changes. However, other questions, such as the association between primary sensory cortex activity and phenomenological content can be studied in healthy and non-clinical, schizophrenia-related subjects, for example nonclinical subjects that show a high loading of schizotypal traits, or close relatives of schizophrenia patients. 


\section{Samenvatting}

In dit proefschrift is de vraagstelling van de neurale en cognitieve substraten van auditieve verbale hallucinaties (AVH) in schizofrenie gesteld als een multivariaat probleem. De functioneel-anatomische substraten worden onderzocht met behulp van functionele magnetische resonantie beeldvorming (fMRI), waarbij de hersenactiviteit non-invasief kan worden gemeten wanneer patiënten hallucinaties ervaren. De functionele data is daarna geanalyseerd met behulp van recentelijk ontwikkelde multivariate statistische technieken, en waar een eerste poging is gemaakt om de functionele data onafhankelijk te analyseren van de hallucinatie zelf rapportages van de patiënten.

\section{Hallucinaties en connectiviteit}

Hoofdstuk 1 introduceert spatiële ICA (sICA) als een techniek om functionele connectiviteit te schatten in fMRI data in het geval dat er geen a priori model voor hersenactiviteit voorhanden is. Functionele connectiviteit is gedefinieerd als de correlatie van de activiteit tussen een set van spatieel gescheiden hersengebieden. SICA werd toegepast op functionele data dat is vergaard terwijl proefpersonen geen enkele vooraf opgegeven taak uitvoerden, vaak aangeduid als de rust status. Deze status speelt een belangrijke rol in functionele beeldvorming studies in het algemeen, omdat de rust status vaak als baseline of controle conditie wordt gebruikt, die dan met de experimentele condities wordt gecontrasteerd, waarbij wordt aangenomen dat tijdens rust het brein geen activiteit vertoond. Tegelijkertijd is het moeilijk dergelijke data te analyseren, ondat er geen vooraf opgesteld model voor hersenactiviteit voorhanden is. SICA is prima geschikt voor de analyse van dergelijke data, omdat het niet noodzakelijk is een model voor hersenactiviteit te specificeren, en omdat het kan worden toegepast op het gehele brein tegelijkertijd. De studie in hoofdstuk 1 laat zien dat tijdens rust het brein activiteit vertoont met een structuur in zowel ruimte als tijd. Binnen functioneel specifieke sensorische en motorische gebieden, zoals de visuele, auditieve en motor cortex, is activiteit in hoge mate gekoppeld terwijl er geen taak wordt uitgevoerd, en is er een sterke contributie van lage tot zeer lage frequenties aan de activiteit. De ruimtelijke en tijdsstructuur van deze gebieden kruist de hemisferen, waar functioneel en anatomisch homologe gebieden van de twee hemisferen in hoge mate 


\section{Samenvatting}

afhankelijk van elkaar zijn. Daarnaast werden dergelijke structuren ook gevonden voor supramodale corticale gebieden in de frontale en temporele kwabben, die zijn gerelateerd aan aandachts en "monitoring" (toezichthoudende of controlerende) processen. Deze resultaten geven aan dat de rust status een hoge mate van ruimtelijke en temporele structuur bezit, wat niet alleen consequenties heeft voor onderzoek naar het "brein in rust", maar ook voor het gebruik van de rust status als contrast voor taak-gerelateerde activiteit.

Hoofdstuk 2 presenteert een eerste applicatie van sICA op functionele data van AVH. In deze studie is het patroon van activiteit tijdens AVH binnen de primaire auditieve cortex onderzocht. De functionele data werd geanalyseerd op een manier die de restricties vermindert van een model voor hersenactiviteit dat is gebaseerd op zelf rapportages van patiënten. Nadat sICA de functionele data in een set van spatiële componenten had ontleed, werden de tijdssieries van de data die geassocieerd waren met de auditieve componenten gevisualiseerd met behulp van BOLD-image grafieken. BOLD-image grafieken maken het voor de onderzoeker mogelijk om de hoeveelheid variabiliteit van single-trial tijdsverlopen visueel te bepalen. De studie toont aan dat, voor zowel hallucinaties als voor akoestische stimulatie, de auditieve componenten een hoge mate van variabiliteit vertonen in het begin, de amplitude en de breedte van de hemodynamische respons. Deze bevindingen geven aan dat, voor tenminste een aantal patiënten, de auditieve cortex - inclusief de primaire auditieve cortex, actief kan zijn tijdens de ervaring van AVH.

Hoofdstuk 3 presenteert een uitbreiding van de applicatie van sICA op data van AVH. De studie laat een verhoogde activiteit zien van bilaterale, doch enigszins rechts-gelateraliseerde primaire en secundaire auditieve cortex, wat geldt als een replicatie van de bevindingen van het vorige hoofdstuk. Voorts is er een netwerk gevonden van frontaal-temporaal gebieden, inclusief de inferieur frontale gyrus, superieur temporale gyrus, mediaal frontale gebieden en inferieur parietale cortex, dat verhoogde activiteit vertoonde tijdens hallucinaties en dat links-gelateraliseerd was bij alle patiënten. De rechter middel temporale gyrus was gerepresenteerd in één enkele component, los van de andere componenten van interesse. Deze netwerken laten geen verschil zien in timing van het hemodynamische signaal behorende bij het drukken op de knop.

In hoofdstuk 4 is de kwestie behandeld van uitvoering en interpretatie van. sICA ontleding over proefpersonen, waarbij een methode is gebruikt dat de spatieel-temporele informatie van individuele proefpersonen bewaard blijft. De toepassing is gericht op de identificatie van componenten van functionele connectiviteit die mogelijk geassocieerd kunnen worden met verschillende anndachts systemen. In deze studie moesten proefpersonen reageren wanneer ze een willekeurig en zeldzaam aangeboden stimulus detecteerden, te midden van een reeks vaak gepresenteerde (taak-irrelevante) stimuli. Proefpersonen zagen ook een zeldzaam gepresenteerde maar taak-irrelevante stimulus, waarbij geen respons nodig was. SICA is gebruikt om de individuele data te 
ontleden in een set van connectiviteits componenten. De individuele resultaten zijn dan samengevoegd (geclusterd) over proefpersonen, waarbij gebruik werd gemaakt van een hierarchische clustering methode, die de spatiele correlaties tussen componenten gebruikt als cluster criterium. De resultaten bevatten verschillende connectiviteits netwerken die gerelateerd zijn aan 1) de "target" maar niet de afleidende stimulus, 2) de afleidende maar niet de target stimulus, en 3) de target en afleidende stimulli in alle proefpersonen.

\section{Hallucinaties en cognitie}

Hoofdstukken 5 en 6 presenteren de analyse van een set van cognitieve en perceptuele taken, en vragenlijsten over mentale voorstelling en hallucinaties, die afgenomen waren bij paranoïde schizofrenie patiènten en gezonde proefpersonen. De cognitieve taken meten psychomotorische snelheid en gecrystaliseerde intelligentie. De perceptuele taken meten aspecten van perceptuele closure, waarbij proefpersonen visueel objecten of geschreven worden moesten completeren, of geometrische vormen moesten onderscheiden van complexe constellaties van figuren. Proefpersonen moesten ook zelfrapportage vragenlijsten over de levendigheid van hun mentale voorstelling en de predispositie tot hallucineren invullen.

De wetenschappelijke literatuur geeft geen overeenstemming aan over de vraag of hallucinaties een verband houden met toegenomen levendigheid van voorgestelde perceptuele ervaringen. In de studie van hoofdstuk 5 is de relatie tussen hallucinaties en mentale voorstelling onderzocht. Een klein aantal testen voor perceptuele en cognitieve vaardigheden werd gebruikt als covariaten om de relatie verder te specificeren. De resultaten tomen aan dat schizofrenie patiënten in het algemeen een hogere levendigheid van mentale voorstelling rapporteren. Echter, er was geen bewijs voor een lineaire relatie tussen levendigheid van mentale voorstelling en predispositie tot hallucineren. De zelf rapportages van voorstelling en hallucinaties waren niet afhankelijk van psychomotorische snelheid of gecrystalliseerde intelligentie. Ook correleerde de mentale voorstelling scores niet met individuele psychopathologische scores. Deze bevindingen suggereren dat (op zelf-rapportage gebaseerde) toegenomen levendigheid van mentale voorstelling een sterk verband houdt met schizofrenie, onafhankelijk van de mate van hallucinaties.

In hoofdstuk 6 is de data van de studie van hoofdstuk 5 heranalyseerd met behulp van principale componenten analyse. In het bijzonder werden de verschillen in de relaties tussen de test scores van perceptuele en cognitieve vaardigheden tussen de groupen onderzocht. De perceptuele testen bevatten drie testen voor perceptuelle closure, de cognitieve testen bevatten een test voor gecrystalliseerde intelligentie en een test voor psychomotorische snelheid. Van psychometrische modellen voor intelligentie werd de hypothese opgesteld dat de perceptuele closure scores - die ook beschouwd kunnen worden alls aspecten van fluide intelligentie - ongecorreleerd zouden moeten zijn met scores van 


\section{Samenvatting}

gecrystalliseerde intelligentie. De test scores en relaties tussen deze scores van de schizofrenie patièten correleerden met individuele scores voor psychopathologie. De resultaten gaven aan dat terwijl de test scores binnen de gezonde groep de dichotomie van gecrystalliseerde vs. fluîde intelligentie reflecteerden (twee principale componenten), de test scores voor de schizofrenie groep in zijn geheel sterk correleerden (één principale component). Het was onwaarschijnlijk dat medicatie zorgde voor deze verhoogde correlaties. Terwijl enkele van de testen gematigd correleerden met positieve symptomen, correleerde de principale component score het sterkst met negatieve symptomen. Het verschil in de componenten structuur suggereert, dat de variatie in test scores binnen de schizofrenie groep het resultaat is van een enkele (cognitieve) pathologische factor, in plaats van een veelheid van wijd verspreide maar onafhankelijke cognitieve stoornissen. Deze vermeende pathologische factor kan dan voomamelijk zijn gerepresenteerd in negatieve symptomen.

\section{Conclusies en ideeën voor de toekomst}

De belangrijkste bevindingen van dit proefschrift kunnen als volgt worden samengevat. De toepassing van spatiële ICA op fMRI data van hallucinaties verschaft aanvullend inzicht in de neurale activiteit van AVH. Primaire auditieve cortex is niet noodzakelijk een algemene eigenschap van auditieve hallucinaties bij schizofrenie, maar in de gevallen dat het wel voorkomt is het tijdsprofiel niet eenvoudig te modelleren d.m.v. zelf rapportages van begin en duur van de hallucinatoire ervaringen.

Tegelijkertijd is toegenomen levendigheid van mentale voorstelling niet een unieke eigenschap van hallucinaties, maar is het meer tekenend voor (paranoïde) schizofrenie als groep. De geassocieerde cognitieve stoornissen zouden een expressie kunnen zijn van een enkelvoudige pathologische factor, die fenotypisch geuit wordt via negatieve symptomen.

Momenteel zijn er geen beschikbare middelen om hallucinaties los van de subjectieve ervaringen van patiënten te bestuderen. Echter, het gebruik van data-gedreven analyses kan de noodzaak van zelf rapportages bij de studie van dergelijke abnormale percepties verminderen. Het is misschien zelfs mogelijk om dergelijke subjectieve ervaringen te bestuderen zonder zelf rapportages, wat het wellicht mogelijk maakt om hallucinaties met een verminderde bias te bestuderen, en om een grotere groep van patiënten te recruteren. Gelijktijdig kunnen dergelijke toepassingen nieuw licht werpen op de neurale substraten van hallucinaties, evenals de toestand of ziekte gerelateerde functionele processen waarbinnen hallucinaties hun oorsprong vinden. Recente studies hebben data-gedreven technieken toegepast of de rust status van schizofrenie patiënten, en hebben een functioneel patroon van de auditieve cortex gevonden die afwijkt van de rust status in gezonde proefpersonen. 


\section{Samenvating}

Deze resultaten suggereren verschillende nieuwe toekomstige onderzoeksrichtingen. Waarom activeren niet alle hallucinerende patiënten de primaire auditieve cortex? Is een dergelijke activiteit misschien gerelateerd aan de fenomenologische ervaring van de hallucinaties? Verschillende studies hebben aangetoond dat hogere orde, maar niet primaire auditieve cortex actief is bij auditieve mentale voorstelling in gezonde proefpersonen en niethallucinerende patiënten. Wat is dan de rol van de primaire auditieve cortex bij de ervaring van hallucinaties? Op een ander niveau is het ook niet duidelijk wat de relatie is van functionele en anatomische connectiviteit tussen sensorische en hogere orde corticale gebieden met halucinaties in schizofrenie. De combinatie van methodes voor het meten en schatten van functionele en anatomische connectiviteit (bijvoorbeeld, diffusie tensor beeldvorming of DTI) zal mogelijk de weg zijn om dergelijke vragen te beantwoorden. Individuele verschillen in psychopathologie, alsmede het neuropsychologisch en psychosociaal functioneren kunnen verder inzicht verschaffen in de complexe en dynamische aspecten van hallucinaties in het bijzonder, en schizofrenie in het algemeen. Terwijl veel van dergelijke studies zich baseren op het meten van schizofrenie patiënten, kunnen andere vragen, zoals de associatie tussen primaire auditieve cortex activiteit en de fenomenologie van de hallucinaties mogelijk worden onderzocht in gezonde en niet-klinische, schizofrenie geassocieerde proefpersonen, bijvoorbeeld met sterke schizotypische eigenschappen, of familieleden van schizofrenie patiënten. 


\section{Color figures}

\section{Chapter 1.}
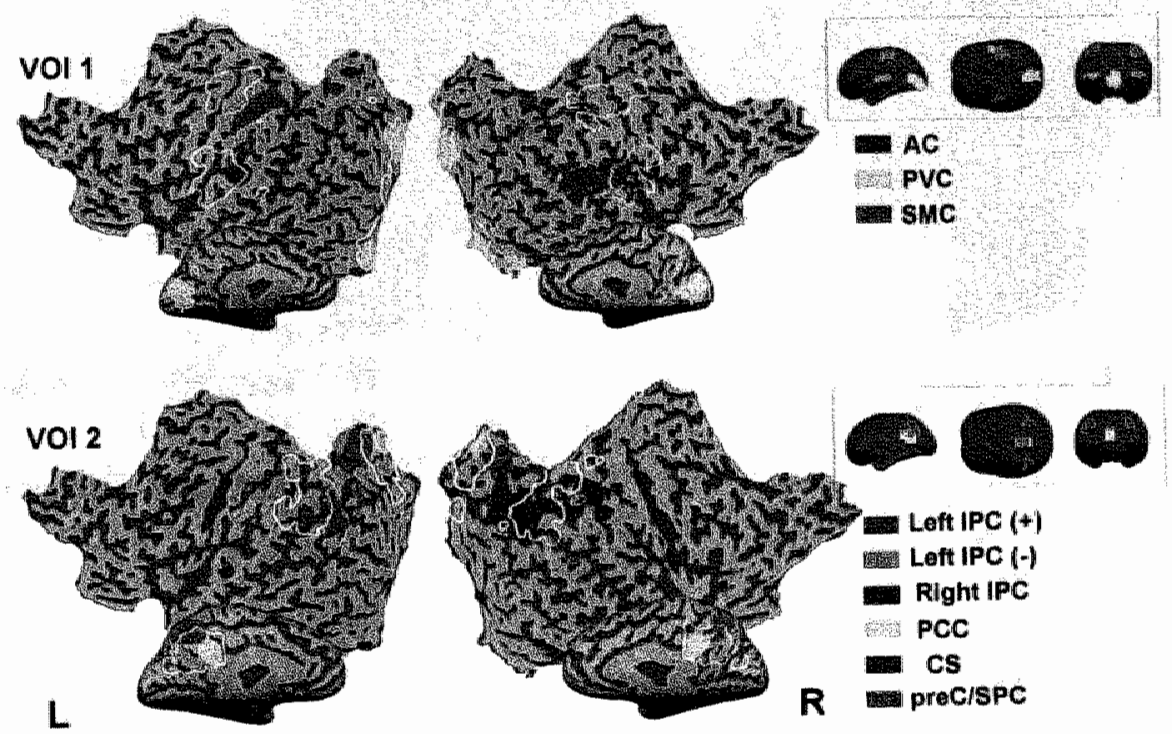

Figure 1.1. 

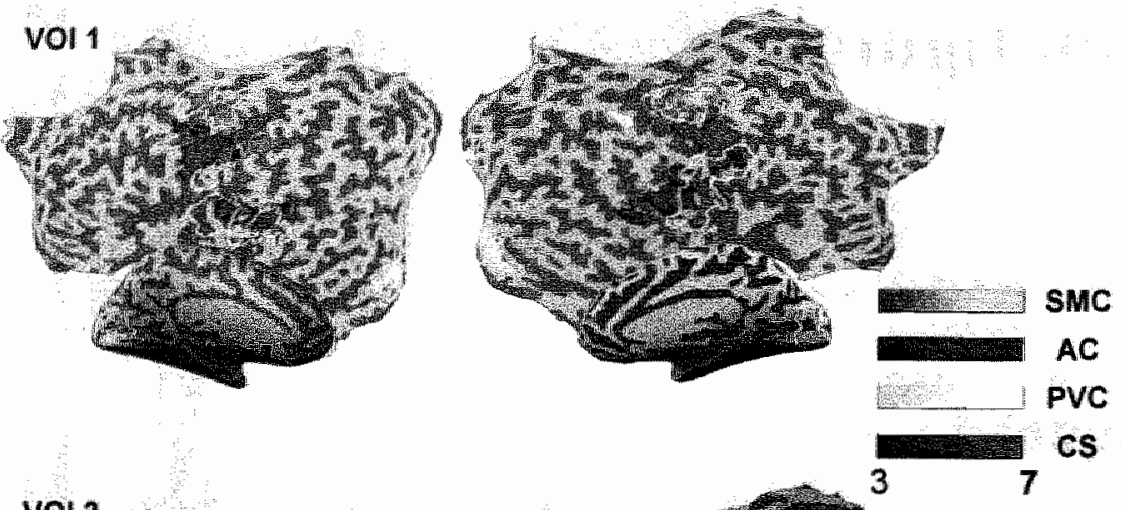

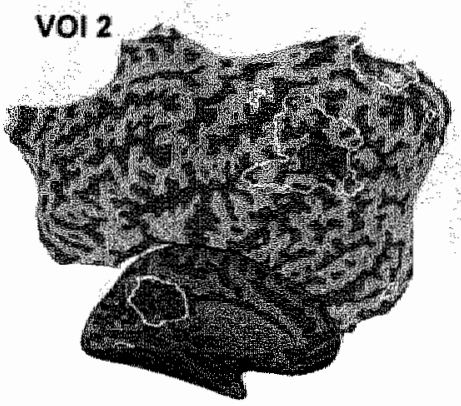

L

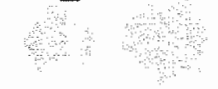

Figure 1.2.

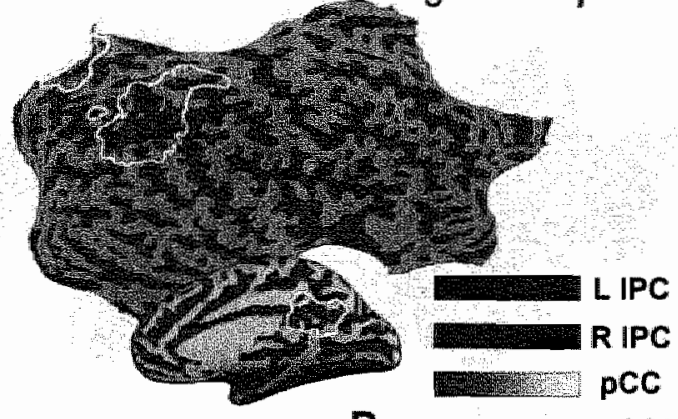

$\mathbf{R}$

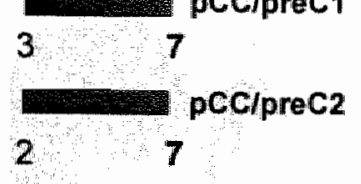

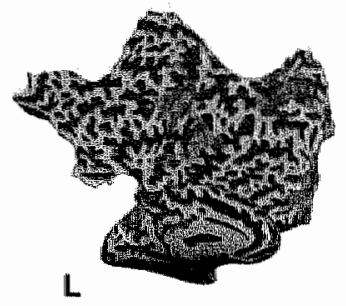

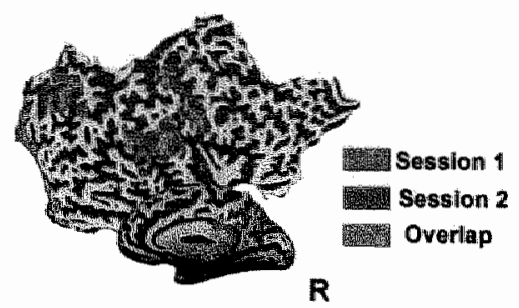

Figure 1.4. 


\section{Color figures}

\section{Chapter 2.}

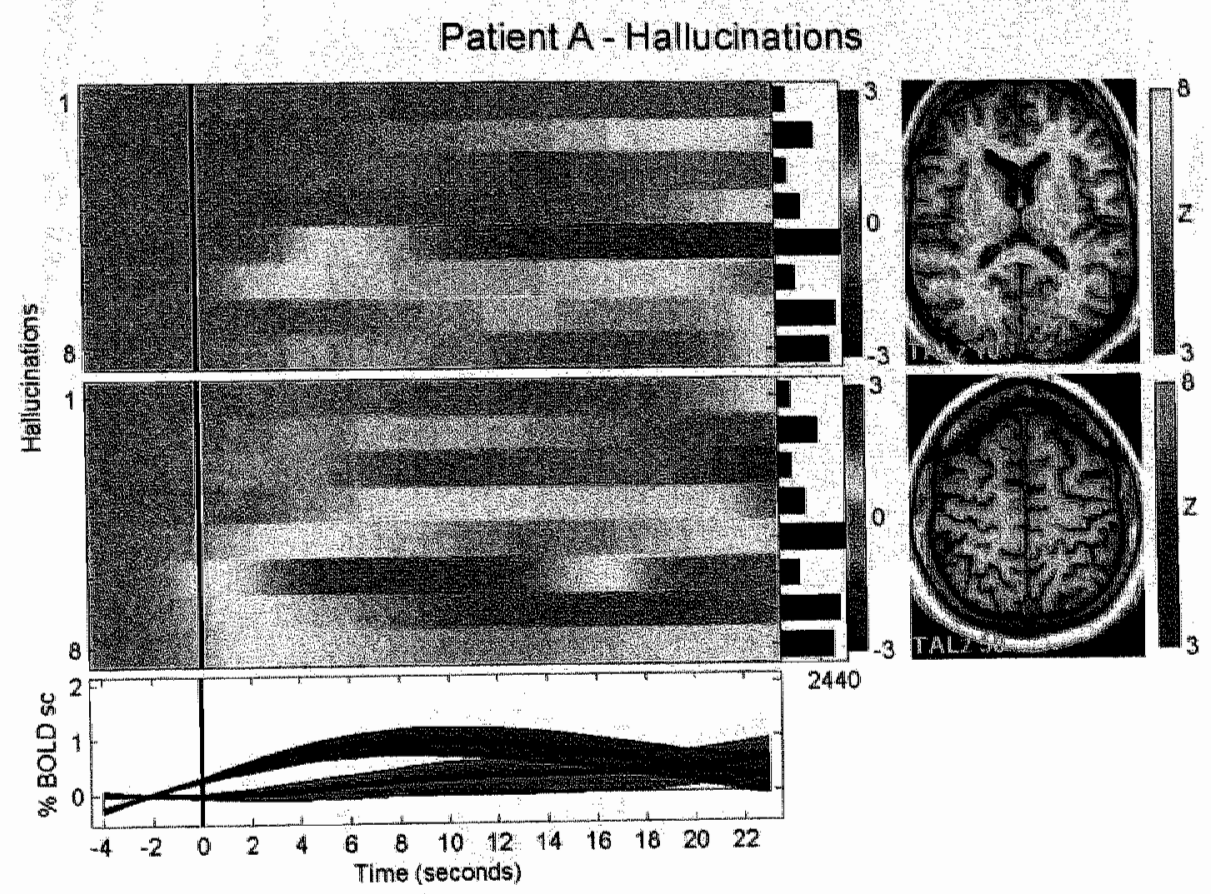

Figure 2.1. 


\section{Patient D - Hallucinations}

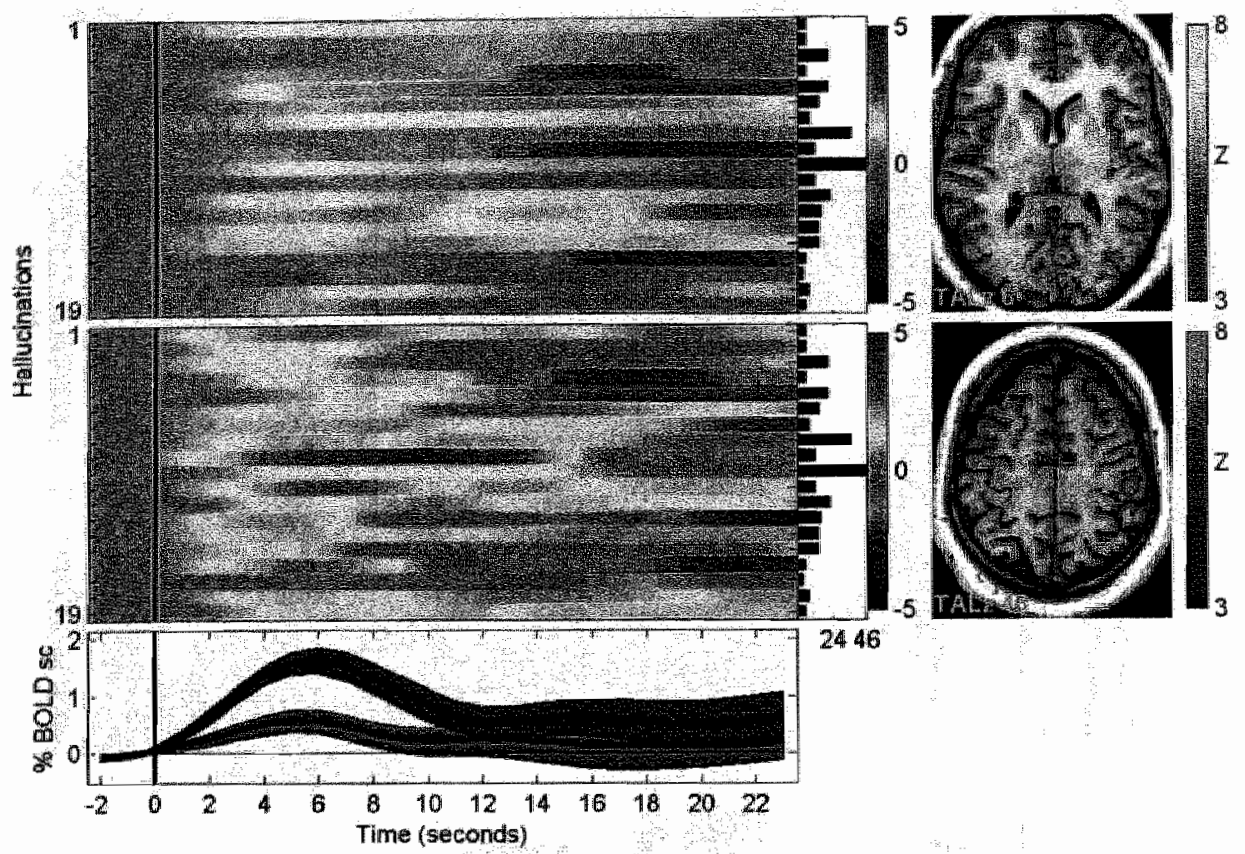

Figure 2.2.

Patient $E$ - Hallucinations

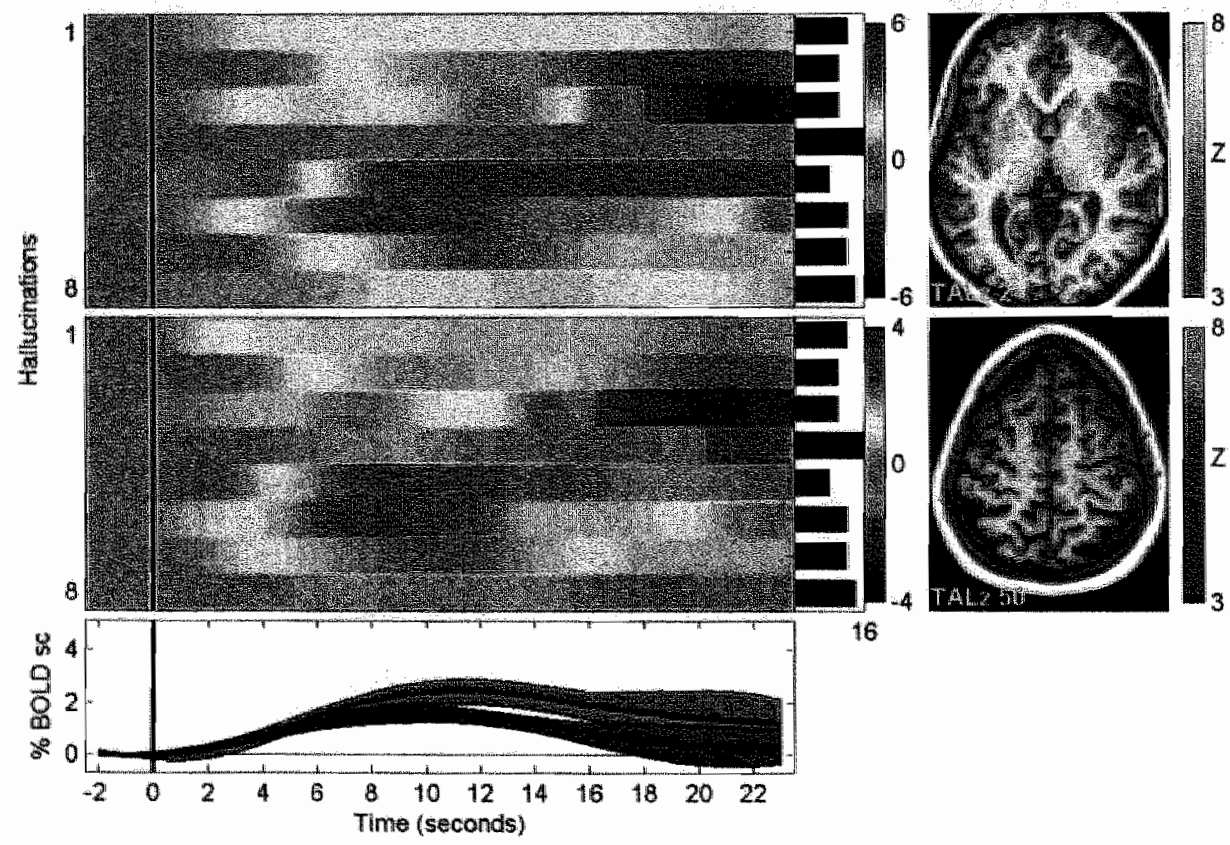

Figure 2.3. 


\section{Colon frgues}
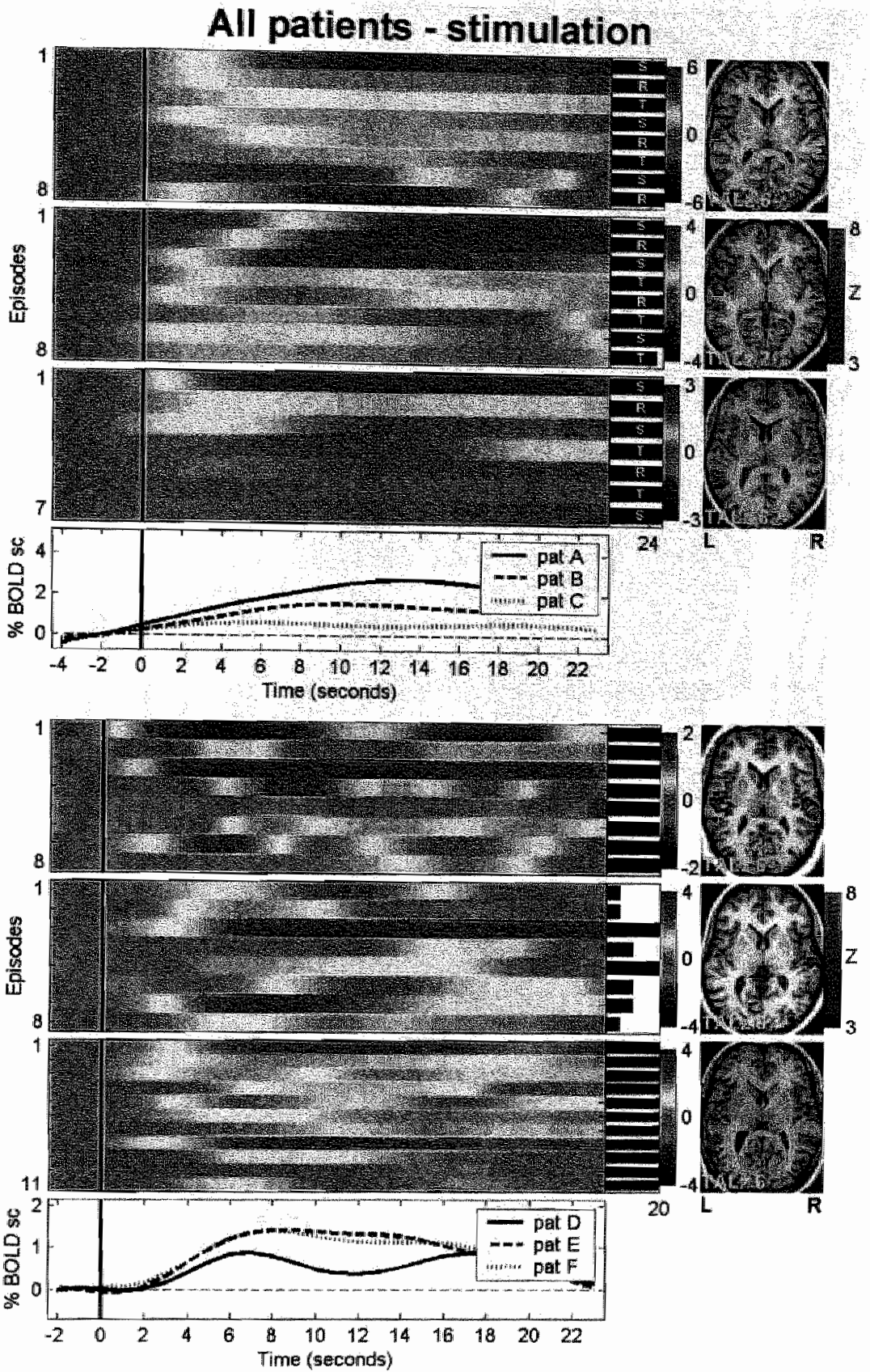

Figure 2.4. 
Color figures
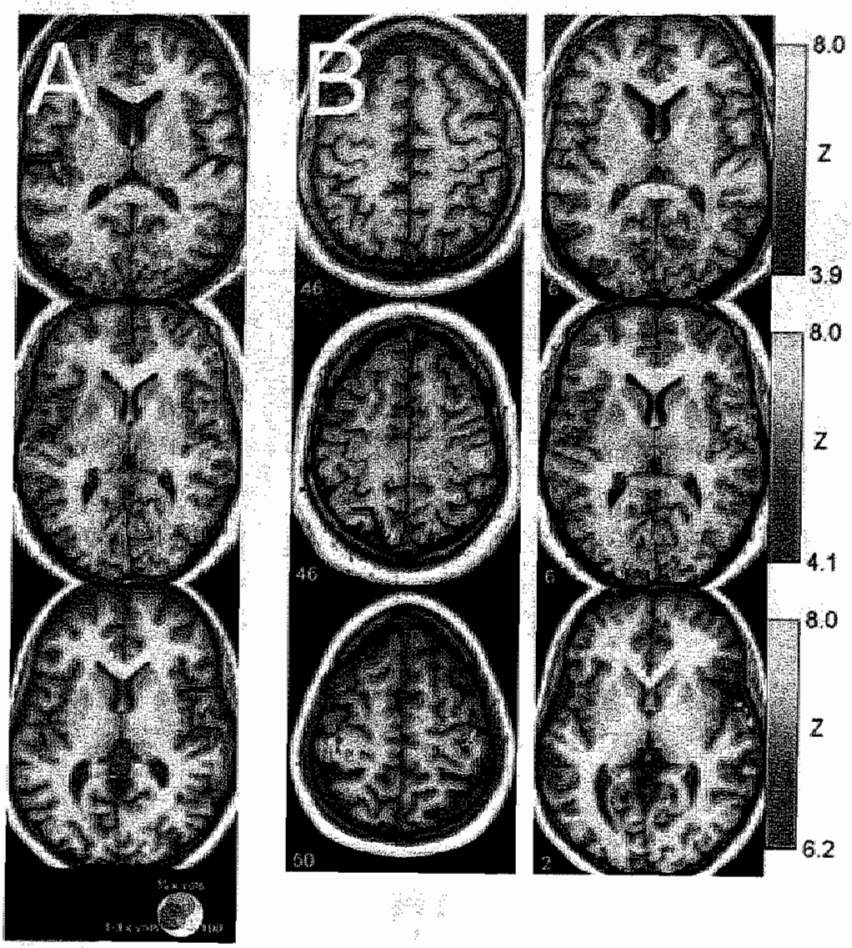

Figure 2.6. 


\section{Color figures}

\section{Chapter 3.}

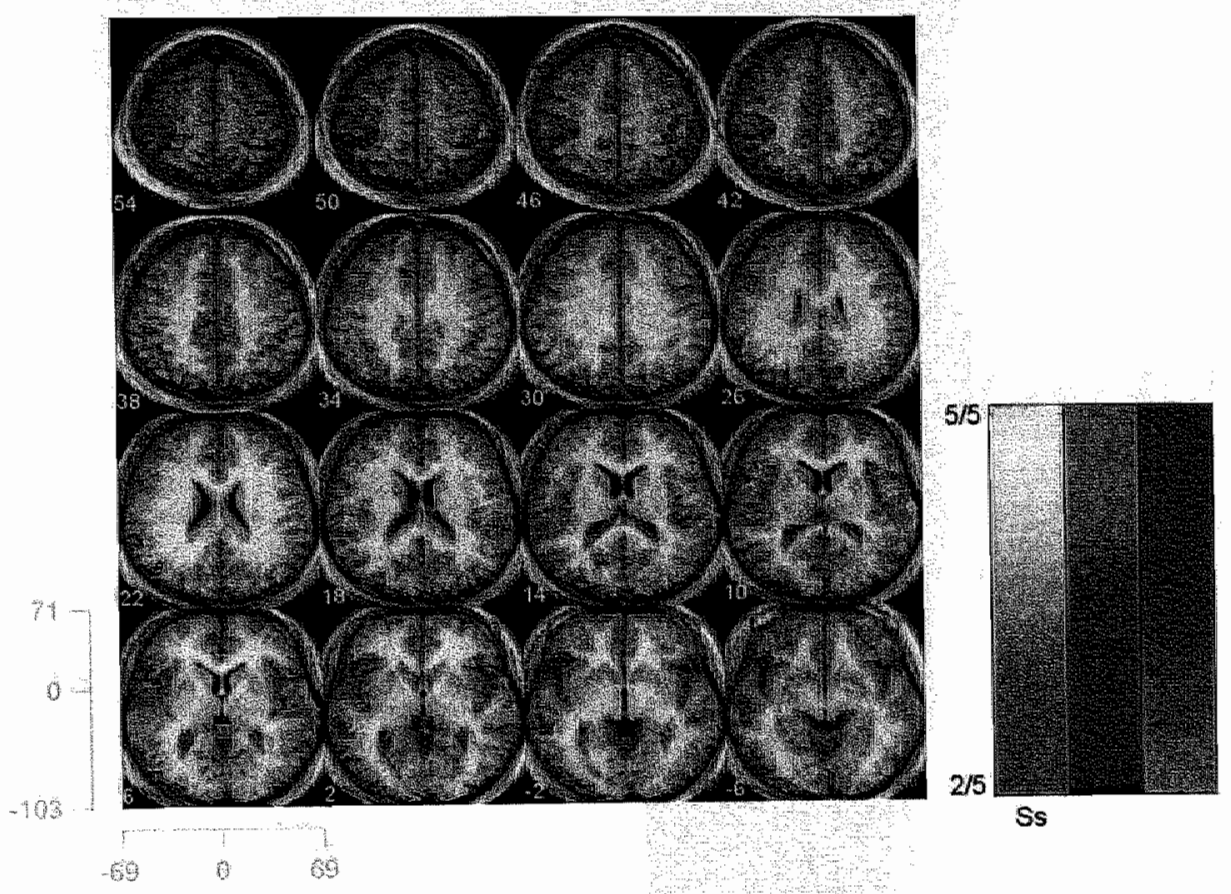

Figure 3.1.
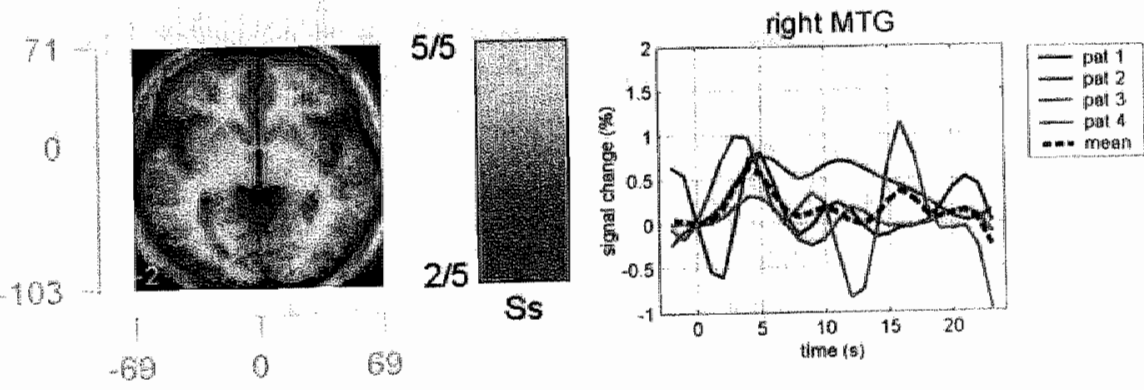

Figure 3.5. (upper row) 


\section{Color figures}

\section{Chapter 4.}

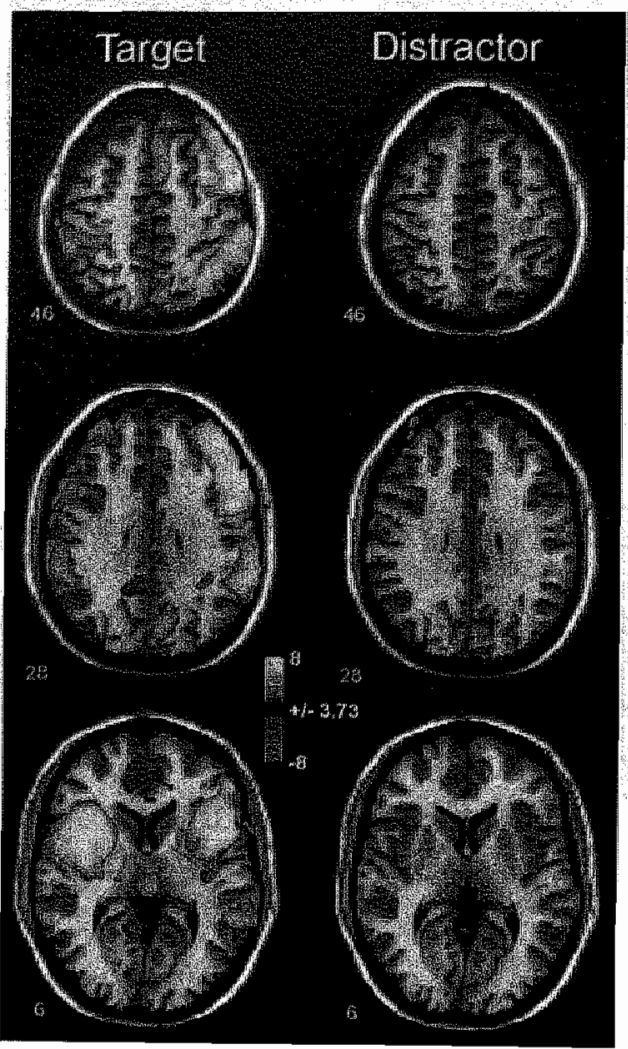

Figure 4.2.

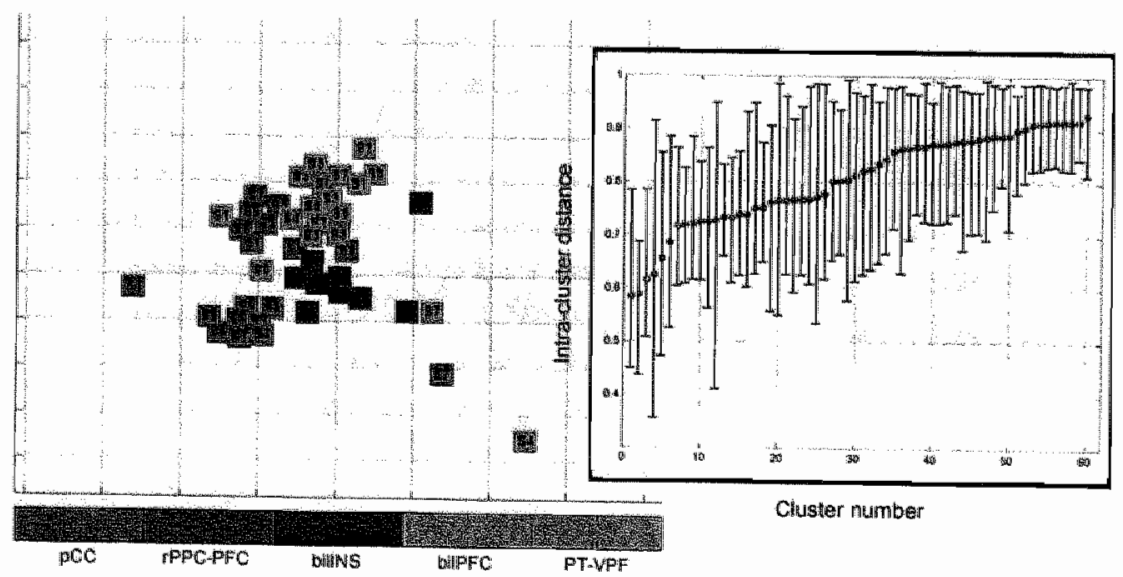

Figure 4.3. 
Colorfigures

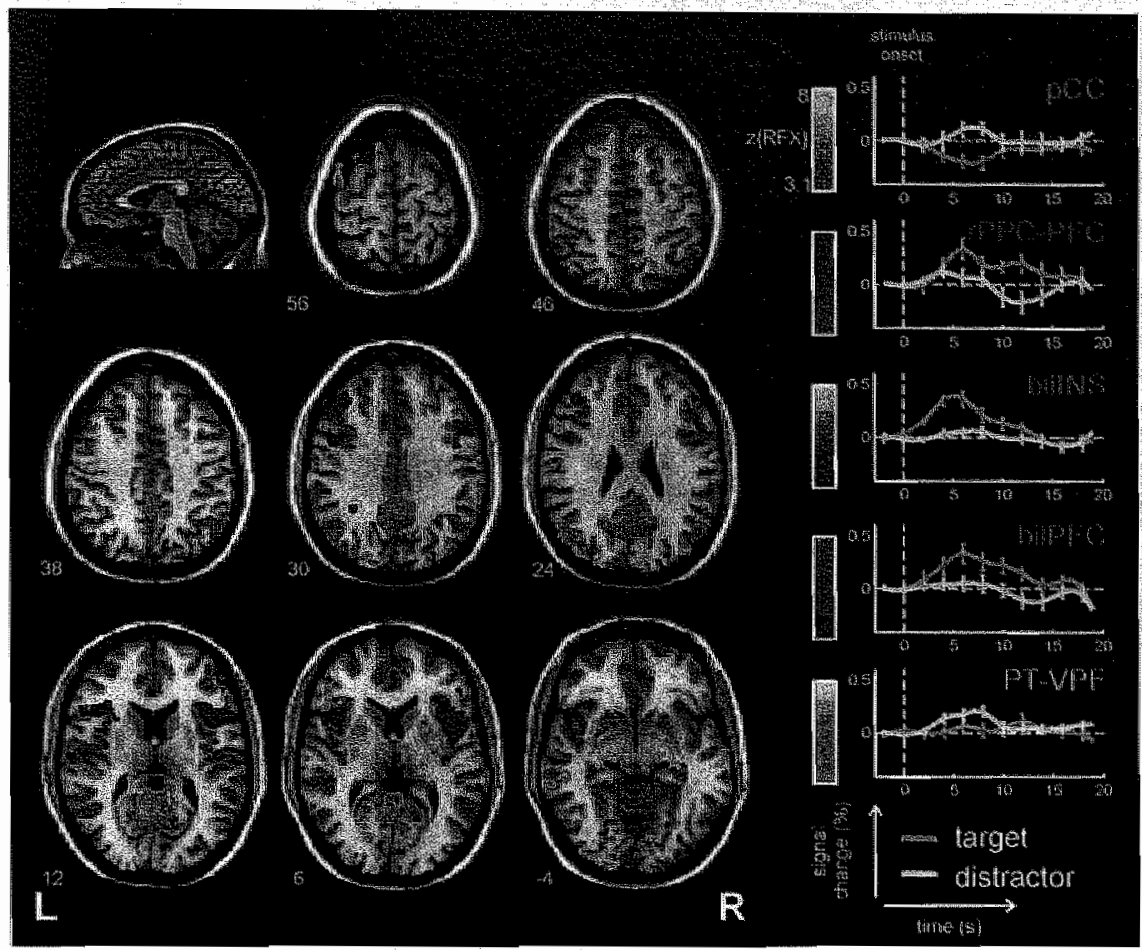

Figure 4.4 .

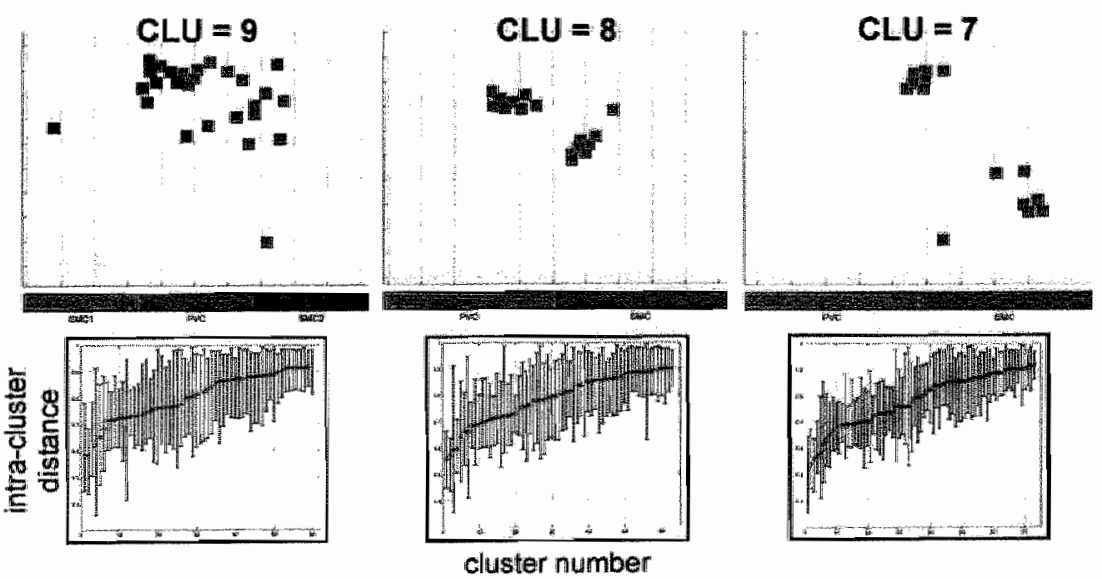

Figure 4.5. 


\section{Color figures}

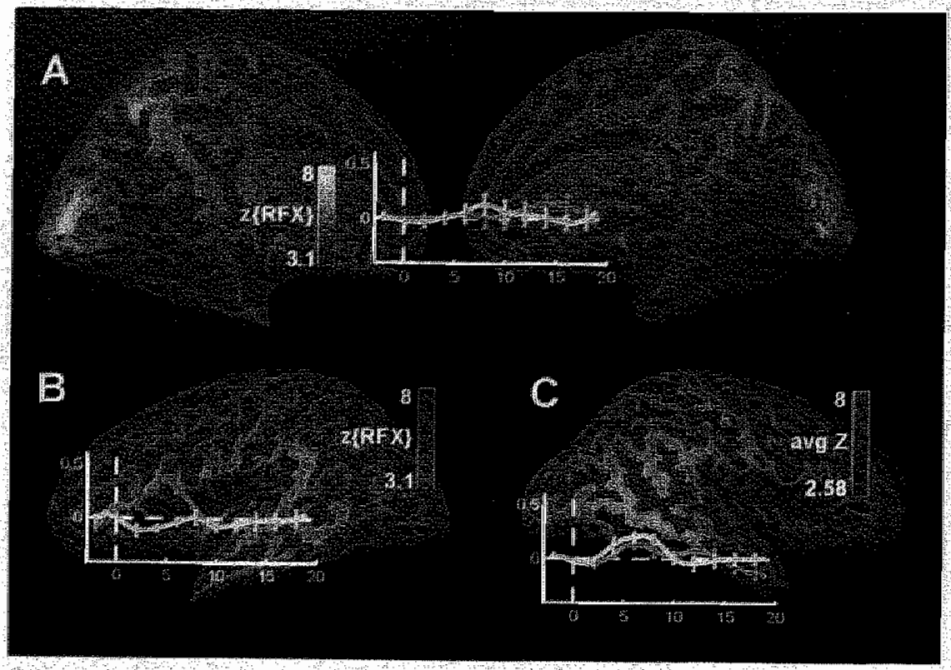

Figure 4.6. 


\section{Abbreviations}

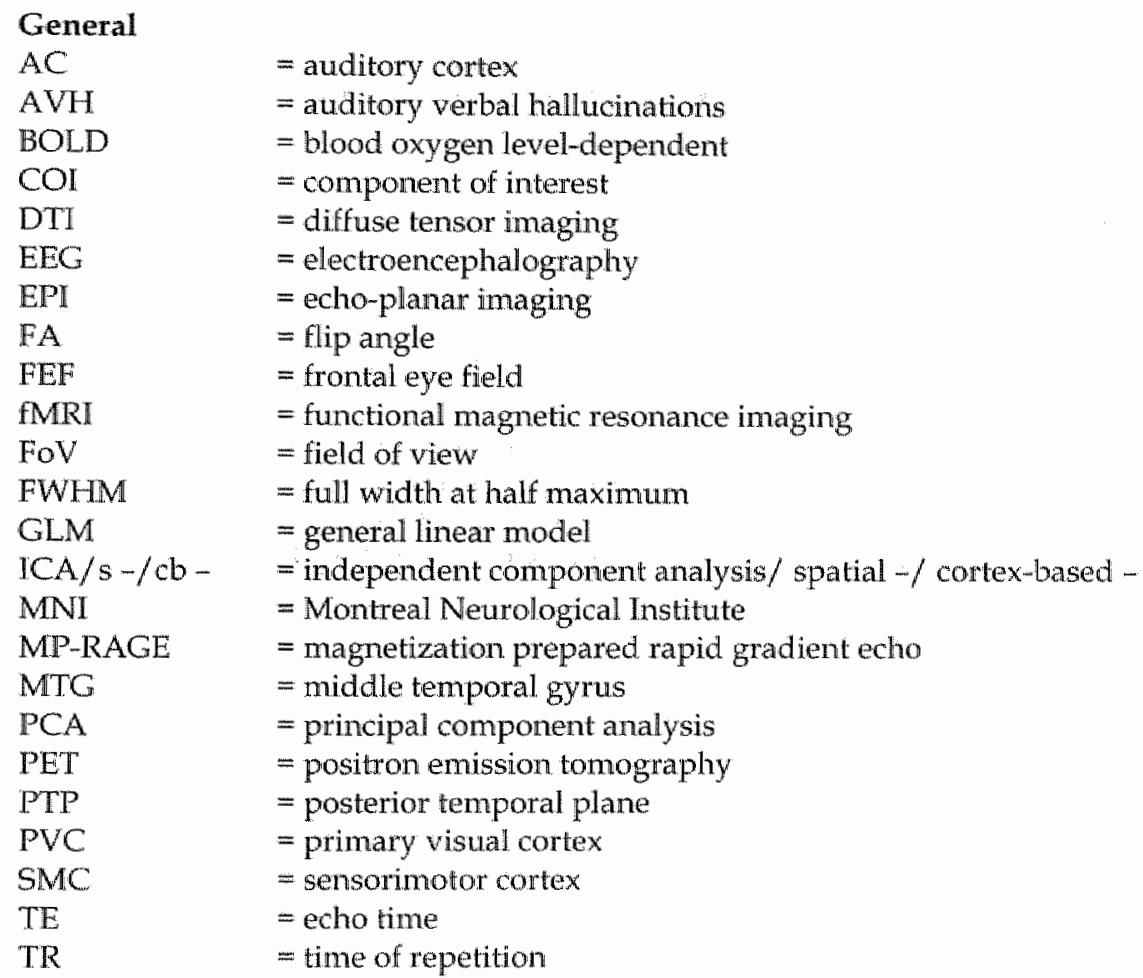

\section{Chapter 1}

CS

$\mathrm{CoM}$

DLPFC

FFT

IPC

IPS

NoV

pCC

preC

$\mathrm{SPC}$

$\mathrm{VOI}(\mathrm{s})$
$=$ central sulcus

= center of mass

= dorsolateral prefrontal cortex

= fast fourier transform

= inferior parietal cortex

= intraparietal sulcus

$=$ number of voxels

= posterior cingulate cortex

= precuneus

= superior parietal cortex

= volume(s) of interest 


\section{Abrewiations}

Chapter 2 \& 3

II

11

$\mathrm{PAC}$

ref

Ss.

\section{Chaplen 4}

binns

BIIPC

CLU

$\mathrm{pCc}$

TI YPF

PPCAEF

sogleA

Chapter $5 \& 6$

\section{ANOVA}

Lis

LSHS

MWT

\section{Cor} $=$ Mehrfach
Comprehension test]

SANS

SAPS

QMI

ZVT'
- fonto temponal

= laterality index

- primary auditory cortex

- reference timecourse

- number of subjects
- bilateral msula component

- bilateral prefrontal component

- cluster size

- posterior cingulate component

- parietal temporal and ventral pretrontal component

= right posterior parietal contex and frontal eye field component

- self-organizing grouping $1 \mathrm{CA}$

$=$ analysis of variance

= Leistungsprufsystem [General Performance Test]

= Launay-Slade Hallucination Scalle

=Mehrfachwahl-Wortschatztest [Multiple Choice Word

- Scale for the Assessment of Negative Symptoms

- Scale for the Assessment of Positive Symptoms

$=\left(\right.$ Bett ${ }^{\prime}$ s shortened) questionnaire upon mental imagery

= Zahlenverbindungstest [Number-Pathfinding test] 


\section{References}

Aleman, A. Böcker, K.B.E. \& de Haan, E.H.F. (1999). Disposition towards hallucinations and subjective versus objective vividness of imagery in normal subjects. Personality and Individual Differences, 27, 707-714.

Aleman, A., Nieuwenstein, M.R. Böcker, K.B.E, \& de Haan, E.H.F. (2001). Multidimensionality of hallucinatory predisposition: factor structure of the LaunaySlade Hallucination Scale in a normal sample. Personality and Individual Differences, $30,287-292$.

Allen, D.N., Huegel, S.G., Seaton, B.E., Goldstein, G., Gurklis Jr., J.A., \& Van Kammen, D.P. (1998). Confirmatory factor analysis of the WAIS-R in patients with schizophrenia. Schizophrenia Reseanch, 34, 87-94.

Aloia, M.S., Gourovitch, M.L., Missar, D., Pickar, D., Weinberger, D.R. \& Goldberg, T.E. (1998). Cognitive substrates of thought disorder, II: Specifying a candidate cognitive mechanism. American Joutnal of Psychiatry, 155, 1677-1684.

American Psychiatric Association (1994). Diagnostic and statistical manual of mental disorders, 4 th ed. Washington, DC: American Psychiatric Press.

An der Heiden, W., \& Häfner, H. (2000). The epidemiology of onset and course of schizophrenia. Enropean Archives of Psychiatry and Clinincal Neuroscience, 250, 292303.

Andreasen, N.C. (1981). Sale for Assessment of Negative Symptoms (SANS). Iowa City: University of Iowa College of Medicine.

Andreasen, N.C. (1984). Scale for Assessment of Positive Symptoms (SAPS). Iowa City: University of lowa College of Medicine.

Andreasen, N.C. (1999). A unitary model of schizophrenia. Archites of Genem Psychatry $56,781-787$.

Ardekani, B.A., Choi, S.l., Hossein-Zadeh, G.A., Porjesz, B., Tanabe, J.L., Lim, K.O., Bilder, R., Helpern, J.A., \& Begleiter, H. (2002). Functional magnetic resonance imaging of brain activity in the visual oddball task. Brain Research Cognitiote Brain Research, 14, 347-356.

Arfanakis, K., Cordes, D., Haughton, V.M., Moritz, C.H., Quigley, M.A., \& Meyerand, M.E. (2000). Combining independent component analysis correlation analysis to probe interregional connectivity in fMRI task activation datasets. Magnetic Resonance Inaging, 18, $921-930$.

Baddeley, A., Emslie, H., \& Nimmo-Smith, 1. (1993). The Spot-the-Word test: a robust estimate of verbal intelligence based on lexical decision. British joumal of Clintical Psycholology, 32, 55-65.

Bandettini, P.A., Jesmanowicz, A., Van Kylen, J., Birn, RM., \& Hyde, J.S. (1998). Functional MRI of brain activation induced by acoustic scanner noise. Magnetic Resonance in Medicine, 39, 410-416. 


\section{Referenices}

Bandettini, P.A.., Jesmamowicz, A., Wong, E.C., \& Hyde, J.S. (1993). Processing strategies for time-course data sets in functional MRI of the human brain. Magnetic Resonance Imaging $30,161-173$.

Barrett, T.R. (1993). Verbal hallucinations in normals, II: Self-reported imagery vividness. Personality and Individual Differences, 15,61-67.

Barrett, T.R., Etheridge, J.B. (1992). Verbal hallucinations in normals, I: People who hear" "voices". Applied Cognitive Psychology, 6, 379-387.

Bartels, A., \& Zeki, S. (2004). Brain dynamics during natural viewing conditions - A new guide for mapping connectivity in wivo. Neurounage, 24,339-349.

Bell, A.J., \& Sejnowski, T.J. (1995). An information-maximisation approach to blind separation and blind deconvolution. Neural Computation, 7, 1129-1159.

Bentaleb, L.A., Beauregard, M., Liddle, P., \& Stip, E. (2002). Cerebral activity associated with auditory verbal hallucinations: a functional magnetic resonance imaging case study. Journal of Psychiatry and Neuroscience, 27, 110-115.

Bentall, R.P. (1990). The illusion of reality: A review and integration of psychological research on hallucinations. Psychological Bulletin, 17, 82 -95.

Bentall, R.P., \& Slade, P.D. (1985a). Reality testing and auditory hallucinations: A signal detection analysis. British joumal of Clinical Psychology, 24, 159-169.

Bentall, R.P., Slade, P.D. (1985b). Reliability of a scale measuring disposition towards hallucination. Persorality and Individual Differences, 6, 527-529.

Berman, I., Viegner, B., Merson, A., Allan, E., Pappas, D., \& Green, A.I. (1997). Differential relationships between positive and negative symptoms and neuropsychological deficits in schizophrenia. Schizophrenia Research, 25, 1-10.

Bichot, N.P., Thompsin, K.G., Rac, S.C., \& Schall, J.D. (2001). Reliability of macaque frontal eye field neurons signaling saccade targets during visual search. Joumal of Neuroscience, 21,713-725.

Binder, J., Albus, M., Hubmann, W., Scherer, I., Sobizack, N., Franz, U., Mohr, F., \& Hecht, S. (1998). Neuropsychological impairment and psychopathology in firstepisode schizophrenic patients related to the early course of illness. European Archives of Psychiatry and Clinical Neuroscience, 248, 70-77.

Biswal, B.B., \& Ulmer, T.L. (1999). Blind source separation of multiple signal sources of fMRI data sets using independent component analysis. Journal of Computer Assisted Tonrography, 23, 265-271.

Biswal, B.B., Yetkin, F.Z., Haughton, V.M., \& Hyde, J.S. (1996). Functional connectivity in the auditory cortex studied with FMRI [abstract]. Nenronnage, 3, 5305.

Biswal, B.B., Yetkin, F.Z., Haughton, V.M., \& Hyde, J.S. (1995). Functional connectivity in the motor cortex of resting human brain using echo-planar MRI. Magnetic Resonance in Medcink, 34, 537-541.

Blakemore, S.J., Smith, J., Steel, R., Johnstone, C.E., \& Frith, C.D. (2000). The perception of self-produced sensory stimuli in patients with auditory hallucinations and passivity experiences: evidence for a breakdown in self-monitoring. $P_{\text {Sycholological }}$ Medicine, 30, 1131-1139.

Bledowski, C., Prvulovic, D., Goebel, R., Zanella, F.E., \& Linden, D.E.J. (2004). Attentional systems in target and distractor processing: A combined ERP and FMRI study. Neuroimage, 22,530-540.

Bleuler, E. (1950). Dementia preacox or the group of solnizophrenias. Zinkin J, translation. International Universities Press, New York

Böcker, K.B.E., Hijman, R., Kahn, R.S., \& De Haan, E.H.F. (2000). Perception, mental imagery and reality discrimination in hallucinating and non-hallucinating schizophrenic patients. British Joumal of Clinical Psychology, 39, 397-406. 
Boynton, G.M., Engel, S.A., Glover, G.H. \& Heeger, DJ. (1996). Linear systems analysis of functional magnetic resonance imaging in human V1. Joumal of Newroscience, 16. $4207-4211$.

Brett, E.A., \& Starker, S. (1977). Auditory imagery and hallucinations. Jom of Nerows and Mental Disense, $164,394-400$.

Brume, M. (2003). Theory of mind and the role of $1 \mathrm{Q}$ in cluronic disorganized schizophrenia. Schizophrenia Research, 60, 57-64.

Cadoret, G., Pike, G.B., \& Petrides, M. (2001). Selective activation of the ventrolateral prefrontal cortex in the human brain during active retrieval processing. Europtent Joumal of Neuroscience, 14, 1164-1170.

Cahill, C., \& Frith, C.D. (1996). A cognitive basis for the signs and symptoms of schizophrenia. In: Pantelis, C., Nelson, H.E., and Barnes, T.R.E., eds. Schizoplinenia: A neuropsychological perspective. London, U.K.: Wiley, pp. $373-395$.

Calhoun, V.D., Adali, T., Pearlson, G.D., \& Pekar, I.J. (2001a). A method for making group inferences from functional MRI data using independent component analysis. Human Brain Mapping, 14, 140-151.

Calhoun, V.D., Adali, T., Pearlson, G.D., \& Pekar, J.J. (2001b). Spatial and temporal independent component analysis if functional MRI data containing a pair of taskrellated waveforms. Human Brain Mapping, 13, 43-53.

Calhoun, V.D., Adali, T., Pekar, J.J. \& Pearlson, G.D. (2003). Latency (in)sensitive ICA group independent component analysis of $\mathrm{ARR}$ data in the temporal frequency domain. Neuroiminge, 20, 1661-1669.

Calhoun, V.D., Kiehl, K.A., Liddle, P.F., \& Pearlson, G.D. (2004). Aberrant localization of synchronous hemodynamic activity in auditory cortex reliably characterizes schizophrenia. Biological Psychiatry, 55, 842-849.

Castelo-Branco, M., Formisano, E., Backes, W., Zanella, F., Neuenschwander, S., Singer, W., Goebel, R. (2002). Activity patterns in human motion-sensitive areas depend on the interpretation of global motion. Proceedings of the Academy of Science USA, 99, 13914-13919.

Chandiramani, K., \& Varma, V.K. (1987). Imagery in schizophrenic patients compared with normal controls. British yournal of Medical Psychology, 60, 335-341.

Childers, D.G. (1978). Modern spectral analysis. New York: IEEE Press.

Clark, V.P., Fannon, S., Lai, S., Benson, R. \& Bauer, L. (2000). Responses to rare visual target and distractor stimuli using event-related fMRI. Joumal of Neurophysiology: $83,3133-3139$.

Comon, P. (1994): Independent component analysis - a new concept? Signal Processing, $36,287-314$.

Corbetta, M. \& Shulman, G.L. (2002). Control of goal-dinected and stimulus-driven attention in the brain. Nature Review Neuroscience, 3, 201-215.

Cordes, D., Haughton, V., Carew, J.D., Arfanakis, K., \& Maravilla, K. (2002). Hierarchical clustering to measure connectivity in AMRI resting-state data. Magnetic Resonance lmaging, 20, 305-317.

Cordes, D., Haughton, V.M., Arfanakis, K., Carew, J.D., Turski, P.A., Moritz, C.H., Quigley, M.A., \& Meyerand, M.E. (2001). Frequencies contributing to functional connectivity in the cerebral cortex in "resting-state" data. American Joumal of Neuroradiology, 22, 1326-1333.

Coull, J.T., Frackowiak, R.S.I, \& Frith, C.D. (1998). Monitoring for target objects: Activation of right frontal and parietal cortices with increasing time on task. Neuropsychologia, $36,1325-1334$. 


\section{References}

Crow, 1.J. (1997). Sehizophrenia as fallure of hemispheric dominance for language. Trends in Neurosiciences, 20, 339-343.

Dickinson, D. lannone, V.N., Wilk, C.M., \& Gold, JM. (2004). General and specific cognilive deficits in schizophrenia. Biological Psychiatry, 55, 826-833.

Dierks, T., Linden, D.E. ., Jand1, M., Formisano, E., Goebel, R., Lanfermann, H. \& Singer, W. (1999). Activation of Heschl's gyrus during auditory hallucinations. Neuron, 22, $615-621$.

Doniger, GM, Silipo, G., Rabinowics, E.F., Snodgrass, I.G., davitt, D.C. (2001). Impaired sensory processing as a basis for object-recognition deficits in schizophrenia. American Jounal of Psychatry, $158,1818-1826$.

Downar, I. Crawley, A.P., Mikulis, D.J. \& Davis, K.D. (2000). A multimodal cortical network of the detection of changes in the sensory environment. Nature Neuroscience, 3, $277-283$.

Duann, J.-R., Jung, T.-P., Kuo, W.-J., Yeh, T.-C., Makeig, S., Hsieh, J.-C., Sejnowski, T.J. (2002). Single-trial variability in event-related BOLD signals. Neuroimage, $15,823-$ 835.

Engelien, A., Huber, W., Sibersweig, D., Stern, E.,Frith, C.D., Dorring, W., et al. (2000). The neural correlates of 'deaf-hearing' in man: Conscious sensory awareness by attentional modulation. Brain, $123,532-545$.

Esposito, F., Formisano, E., Seifritz, E., Goebel, R., Morrone, R., Tedeschi, G., \& Di Salle, F. (2002). Spatial independent component analysis of functional MRI time-series: To what extent do results depend on the algorithm used? Human Brain Mapping, 16, 146-157.

Esposito, F., Scarabino, T., Hyvärinen, A., Himberg, J „Formisano, E., Comani, S., Tedeschi, G., Goebel, R., Seifritz, E., \& Di Salle, F. (2005). Independent component analysis of $\mathrm{EMRI}$ group studies by self-organizing clustering. Neuroinage, 25, 1.93. 205.

Fifytche, D.H., Howard, R.J., Brammer, M.J., David, A., Woodruff, P., \& Williams, S. (1998). The anatomy of conscious vision: An FMRI study of visual hallucinations. Nature Neuroscience, $1,738-742$.

Formisano, E., Esposito, F., Di Salle, F., \& Goebel, R. (2001). Cortex-based independent component analysis of ARI time-series. Neuroimage, $13,5119$.

Formisano, E., Esposito, F., Di Salle, F., \& Goebel, R. (2004). Cortex-based independent component analysis. Magrkekic Resonance Inaging, 22, 1493-1504.

Formisano, E., Esposito, F., Kriegeskorte, N., Tedeschi, G., Di Salle, F, \& Goebel, R. (2002). Spatial independent component analysis of functional magnetic resonance imaging time-series: Characterization of the cortical components. Neuroconnuting. $49,241-254$.

Formisano, E., Goebel, R. (2003). Tracking cognitive processes with functional MRI mental chronometry. Current Opinion in Neurobiology, 13, 174-181.

Formisano, E., Kim, D.-S., Di Salle, F., Van de Moortele, P.-F., Ugurbil, K., \& Goebel, R. (2003). Mirror-symmetric tonotopic maps in human primary auditory cortex. Neuron, 40, 859-869.

Fries, P., Neuenschwander, S., Engel, A.K., Goebel, R., \& Singer, W. (2001). Rapid feature selective neuronal synchronization through correlated latency shifting. Nature Neuroscience, $4,194-200$.

Friston, K.J. (1996). Statistical parametric mapping and other analyses of functional imaging data. In: Toga AW, Mazziotta $\mathrm{JC}$, editors. Brain mapping: The methods. San Diego: Academic Press. p 363-396.

Friston, K.]. (1998). The disconnection hypothesis. Schizoplwenia Research, 30, 115-125. 
Friston, K.J., Frith, C.D., Liddle, P.F. \& Frackowiak, R.S. (1993). Functional connectivity: The principle-component analysis of large (PET) data sets. Jowmal of Cerebral Blood Flow and Metabolism, 13,5-14.

Gaeta, H., Friedman, D. \& Hunt, $\mathrm{G}$. (2003). Stimulus characteristics and task category dissociate the anterior and posterior aspects of the novelty P3. Psychophysiology, 40, 198-208.

Galton, F. (1883). Inquiries into human faculty amd its development. London: MacMillan \& Co.

Gaser, C., Nenadic, I., Volz, H-P., Büchel, C., \& Sauer, H. (2004). Neuroanatomy of "hearing voices": A frontotemporal brain structural abnormality associated with auditory hallucinations in schizophrenia. Cetebral Cortex, 14,91-96.

Genovese, C.R., Lazar, N.A., \& Nichols, T. (2002). Thresholding of statistical maps in functional neuroimaging using the false discovery rate Newroimage, 15, 870-878.

Goebel, R., Linden, D.E.J., Lanfermann, H., Zanella, F.E, \& Singer, W. (1998). Functional imaging of mirror inverse reading reveals separate coactivated networks for oculomotion spatial transformations. Neuroreport, 9, 713-719.

Goldstein, A., Spencer, K.M., \& Donchin, E. (2002). The influence of the stimulus deviance and novelty on the P300 and nowelty P3. Psychophysiology, 39, 713-719.

Gottesman, I.I. \& Gould, T.D. (2003). The endophenotype concept in psychiatry: Etymology and strategic intentions. American foumal of Psychiatry, 160, 636-645.

Green, C., \& Cordes, D. (2002). Preprocessing functional MRI data with principal component analysis adversely affects the results of independent component analysis [abstract]. Neurointige, 16, 5357 .

Greicius, M.D., Krasnow, B., Reiss, A.L., \& Menon, V. (2003). Functional connectivity in the resting brain: A network analysis of the default mode hypothesis. Procedings of the National Academy of Science USA, 100, 253-258.

Gu, H., Engelien, W., Hanhua, F., Silbersweig, D.A., Stern, E., \& Yang, Y. (2001). Mapping transient, randomly occurring neuropsychological events using independent component analysis. Neuroimage, 14, 1432-1443.

Gusnard, D.A., \& Raichle, M.E. (2001). Searching for a baseline: Functional imaging and the resting human brain. Nature Rexiew Neuroscience, 2, 685-694.

Halpern, A.R., \& Zatorre, R.J. (1999). When that tune runs through your head: A PET investigation of auditory imagery of familiar melodies. Cerebral Cortex, 9, 697-704.

Hampson, M., Peterson, B.S., Skudlarski, P., Gatenby, J.C., \& Gore, J.C. (2002). Detection of functionall connectivity using temporal correlations in MR images. Human Broin Mapping, 15, 247-262.

Harms, M.P., \& Melcher, J.R. (2003). Detection and quantification of a wide range of fMRI temporal responses using a physiologically-motivated basis set. Hwmin Brin Mapping, 20, 168-183.

Heinrichs, R.W., \& Zakzanis, K.K. (1998). Neurocognitive deficit in schizophrenia: A quantitative review of the evidence. Neuropsychology, $12,426-445$

Henson, R.N.A., Price, C.., Rugg, M.D., Tumer, R., \& Friston, K.J. (2002). Detecting latency differences in event-related BOLD responses: Application to words versus nonwords and initial wersus repeated face presentations. Neturoimage, 15, 83-97.

Himberg, J., Hyvarinen, A., \& Esposito, F. (2004). Validating the independent components of neuroimaging time series via clustering and visualization. Newroinage, 22, 1214- 1222 .

Hirayasu, Y., McCarley, R.W., Salisbury, D.F., Tanaka, S., Kwon, J.S., Frumin, M., Snyderman, D., Yurgelon-Todd, D., Kikinis, R., Joles:., F.A. \& Shenton, M. (2002). 


\section{References}

Planum temporale and Hesch gyrus volume reduction in schizophrenia. Ardives of General Psychatry, $57,692-699$

Hoffman, R.E. \& McGlashan, TH. (1993). Parallel distributed processing the emergence of schizophrenic symptons. Schizophrenia Bulletin, 19, 119-140.

Hopfinger, I.B., Buonocore, M.H. \& Mangun, G.R. (2000). The neural mechanisms of top-down attentional control. Nature Neuroscience, 3, 284-291.

Hom, I.L \& Cattell, R.C. (1966). Refinement and test of the theory of fluid and crystallized general intelligences. Joumal of Educational Psychology, 57, 253-280.

Horn, W. (1962). Leictungsprufsystem (LPS). Gottingen: Verlag für Psychologie.

Horowitz, M. (1975). Hallucinations: An information processing approach. In: Siegel, R.K., and West, LI. eds. Hallucinations: Behavior, experience and theory. New York, NY: Wiley, Pp. 163-196.

Hubl, D., Koenig, T., Strik, W., Federspiel, A., Kreis, R., Boesch, C., Maier, S.E., Schroth, G. Lovblad, $K_{\text {, \& }}$ Dierks, T. (2004). Pathways that make voices: White matter changes in auditory hallucinations. Archues of General Psychiatry, 61, 658-668.

Hywärinen, A. (1999). Fast robust fixed-point algorithms for independent component analysis. IEEE Transactions on Neural Networks, 10, 626-634.

Jancke, L., Shahram, M., \& Shah, N.J. (1999). Attention modulates activity in the primary and the secondary auditory cortex: a functional magnetic resonance imaging study in human subjects. Neuroscience Letters, 266, 125-128.

Jogems-Kosterman, B.J.M., Zitman, F.G., Van Hoof, J.J.M. \& Hulstijn, W. (2001). Psychomotor slowing and planning deficits in schizophrenia. Schizophrenia Research, 48, 317-333.

Jung, T.-P.", Makeig, S., Westerfield, M., Townsend, J., Courchesne, E., \& Sejnowski, T.J. (2001). Analysis and visualization of single-trial event-related potentials. Humart Brain Mapping, 14, 166-185.

Kail, R. (1997), Processing time, imagery, and spatial memory. Journal of Experimental Child Psychology, 64, 67-78.

Ker.ns, J.G., Berenbaum, H, Barch, D.M., Bartich, M.T., \& Stollar, N. (1999). Word production in schizophrenia and its relationship to positive symptoms. Psychtatry Research, 87, 9-37.

Kiehl, K.A., Laurens, K.R., Duty, T.L., Forster, B.B., \& Liddle, P.F. (2001). Neural sources involved in auditory target detection and novelty processing: An event-related RMRI study. Psychophysiology, 38, 133-142.

Kim, I., Glahn, D.C., Nuechterlein, K.H., \& Cannon, T.D. (2004). Maintenance and manipulation of information in schizophrenia: Further evidence for impairment in the central executive component of working menory. Schizophrenin Research, 68, $173-187$.

Kiviniemi, V., Kantola, J.H., Jauhiainen, J., Hyvarinen, A., \& Tervonen, O. (2003). Independent component analysis of nondeterministic fMRI signal sources. Neuroimage, 19, 253-260.

Koch, M.A. Norris, D.G., \& Hund-Georgiadis, M. (2002). An investigation of functional anatomical connectivity using magnetic resonance imaging. Neuroimage, 16, 241250.

Kok, A. (2001). On the utility of P3 amplitude as a measure of processing capacity. Psychophysiology, 38, 557-577.

Kosslyn, S.M. (1994). Innge and brain. Cambridge, MA: MrT Press.

Kriegeskorte, N., \& Goebel, R. (2001). An efficient algorithm for topologically correct segmentation of the cortical sheet in anatomical MR volumes. Newroimage, 14, 329346. 


\section{References}

Launay, G., Slade, P.D. (1981). The measurement of hallucinatory predisposition in male and female prisoners. Personwity and Imdividual Differences, 2, 221-234.

Lawrence, N.S. Ross, T.J., Hoffmann, R., Garavan, H., \& Stein, E.A. (2003). Multiple neuronal networks mediate sustained attention. foumal of Cognitioe Newrosciento, 15 , $1028-1038$.

Lawrie, 5.M. Büchel, C, Whalley, H.C., Frith, C.D., Friston, K., \& Johnstone, E.C. (2002). Reduced frontotemporal functional connectivity in schizophrenia associated with auditory hallucinations. Biological Psychiatry, 51, 1008-1011.

Lehrl, S. (1989). Mehrfachwahl-Wortschatz-Intelligenztest MWT-B. Erlangen: Perimed.

Lennox, B.R., Park, S.B., Jones, P.B., Morris, P.G. \& Park, G. (1999). Spatial and temporal mapping of neural activity associated with auditory hallucinations. Lancet, 353, 644 .

Lennox, B.R. Park, S.B., Medley, I., Morris, P.G., \& Jones, P.B. (2000). The functional anatomy of auditory hallucinations in schizophrenia. Psychiatry Research $100,13-20$.

Leopold, D.A., Murayama, Y., \& Logothetis, N.K. (2003). Very slow activity fluctuations in monkey visual cortex: Implications for functional brain imaging. Cerebral Cortex, $13,422-433$.

Levitan, C., Ward, P.B., Catts, S.V., \& Hemsley, D.R. (1996). Predisposition toward auditory hallucinations: the utility of the Launay-Slade Hallucination Scale in psychiatric patients. Personality and Individual Differences, 21, 287-289.

Lewis, D.A., \& Levitt, P. (2002). Schizophrenia as a disorder of neurodevelopment. Anmual Review Neuroscience, $25,409-432$.

Linden, D.E.J., Prvulovic, D., Formisano, E., Voellingew, M., Zanella, E.E., Goebel, R., \& Dierks, T. (1999). The functional neuroanatomy of target detection: An AMRI study of visual and auditory oddball tasks. Cerebra! Cortex, 9, 815-823.

Lobban, F., Haddock, G., Kinderman, P.\& \& Wells, A. (2002). The role of metacognitive beliefs in auditory hallucinations. Personality and Individual Differences, 32, 13511363.

Logothetis, N.K., Pauls, J., Augath, M., Trinath, T. \& Oeltermann, A. (2001). Neurophysiological investigation of the basis of the fMRI signall. Nature, 412, 150157.

Lowe, M., Philips, M.D., Lurito, J.T., Mattson, D., Dzemidzic, M., \& Mathews, V.P. (2002). Multiple sclerosis: Low-Frequency temporall Blood Oxygen LevelDependent fluctuations indicate reduced functional connectivity - initial results. Radiology, 224, 184-192.

Lowe, M.J., Mock, B.J., \& Sorenson, J.A. (1998). Functional connectivity in single multislice echoplanar imaging using resting-state fluctuations. Neuroinage, 7,119 132.

McGuire, P.K., Silbersweig, D.A., \& Frith, C.D. (1996a). Funclional neuroanatomy of verbal self-monitoring. Brain, 119, 907-917.

McGuire, P.K., Silbersweig, D.A., Wright, I., Murray, R.M., Frackowiak R.S, \& Frith, C.D. (1996b). The neural correlates of inner speech and auditory verbal. imagery in schizophrenia: relationship to auditory verbal hallucinations. British fournal of Psychiatry, 169,148-59.

McKay, C.M., Headlam, D.M., \& Copolov, D.L. (2000). Central auditory processing in patients with auditory hallucinations. Awterican Journal of Psychiatry, 157, 759-766.

Mckeown, M.J., \& Sejnowski, T.I. (1998). Independent component analysis of AMRI data: examining the assumptions. Human Brain Mapping, 6, 368-372.

Mckeown, M.J., Jung, T.-P., Makeig, S., Brown, G., Kindermann, S.S.r Lee, T.-W., Sejnowski, T.J., (1998a). Spatially independent activity patterns in functional MRI 
data during the Stroop color-naming task. Procedings of the National Academy of Science USA, 95, 803-810.

Mckeown, M. , Makeig, S., Brown, G.G., Jung, T.P., Kindermann, S.S., Bell, A.J., \& Sejnowski, T.]. (1998b). Analysis of AMRI data by blind separation into independent spatial components. Human Brain Mapping, 6, 160-188.

Mintz, S, \& Alpert, M. (1972). Imagery vividness, reality testing, and schizophrenic hallucinations. Journal of Abnomal Psychology, $79,31-316$

Mitra, P.P., Ogawa, S. Hu, $X$, \& Ugurbil, K (1997). The nature of spatiotemporal changes in cerebral hemodynamics as manifested in functional magnetic resonance imaging. Magnetic Resonance in Medicine, 37, 511-518.

Mohamed, S., Paulsen, J.S. O'Leary, D., Annd, S. \& Andreasen, N. (1999). Generalized cognitive deficits in schizophernia: A study of first-episode patients. Archives of General Psychiatry, 56, 749-754.

Moritz, C.H., Rogers, B.P., \& Meyerand, M.E. (2003). Power spectrum ranked independent component analysis of a periodic AMRI complex notor paradigm. Human Brain Mapping, 18, 111-122.

Muggleton, N.G., \uan, C.-H. Cowey, A., \& Walsh, V. (2003). Human frontal eye fields and visual search. Journal of Neurophysiology, 89, 3340-3343.

Munk, M.H.J., Linden, D.E.J., Muckli, L., Lanfermann, H., Zanella, $\mathbb{E}$.E., Singer, W., \& Goebel, R. (2002). Distributed cortical systems in visual short-term memory revealed by event-related functional magnetic resonance imaging. Cerebral Cortex, $12,866 \cdots 876$.

O'Shea, J., Muggieton, N.G., Cowey, A., \& Walsh, V. (2004). Timing of target discrimination in human frontal eye fields. Jownal of Cognitive Neuroscience, 16, 1060-1067.

Obrig, H., Neufang, M., Wenzel, R., Kohl, M., Steinbrink, J, Einhäupl, K., \& Villringer, A. (2000). Spontaneous low frequency oscillations of cerebral hemodynamics and metabolism in human adults. Neuroinage, 12, 623-39.

Oswald, W.D. \& Roth. E. (1987). Der Zahlen-Verbindungs-Test ZVT. Göttingen: Hogrefe.

Poeppel, D., \& Hickok, G. (2000). Towards a Functional Anatomy of Speech Perception. Trends in Cognitive Sciences, 4, 131-138.

Pratt, W. (1991). Digital Image Processing (2nd ed.). New York: Wiley.

Raczkowski, D., Kalat, J.W., \& Nebes, R. (1974). Reliability validity of some handedness questionnaire itens. Neuropsychologia, 6,4347 .

Rademacher, J., Morosan, P., Schormann, T., Schleicher, A., Werner, C., Freund, H.-J., \& Zilles, K., (2001). Probabilistic mapping and volume measurement of human primary auditory cortex. Neuromage, 13, 669-683.

Raichle, M.E., Macleod, A.M., Snyder, A.Z., Powers, W.J., Gusnard, D.A., \& Shulman, G.L. (2001). A default mode of brain function. Proceedings of the National Academy of Science USA, 98, 676-682.

Reitan, R.M. (1956). Trail Making Test. Manual for ad mintistration, scoring and interpretation. Indianapolis: lndiana University Press.

Sack, A.T., Van de Ven, V.G., Etschenberg, S., Schatz, D., \& Linden, D.E.I. (2005) Enhanced vividness of mental imagery as a trait marker for schizophrenia? Schizophrenia Bulletin, 1,97-104.

Schmithorst, V.J., \& Holland, S.K. (2004). Comparison of three methods of generating group statistical inferences from independent component analysis of functional magnelic resonance imaging data. Ioumal of Magnetic Resonance Imaging, 19, 365368. 
Seifritz, E, Esposito, F., Hennel, F., Mustovic, H. Newhoff, J.G., Bilecen, D. et al., (2002). Spatiotemporal pattern of neural processing in the human auditory cortex. Science, 297, 1706-1708.

Sheehan, P.W. (1967). A shortened form of Bett's questionnaire upon mental imagery. Joumal of Clinical Psychology, 23, 386-389.

Shen, B., Nadkarni, M., \& Zappulla, R.A. (1999). Spectral modulation of cortical connections measured by EEG coherence in humans. Cinical Newrophysiology, 110. 115-125.

Shergill, S.S., Brammer, M.J., Williams, S.C., Murray, R.M., \& McGuire, P.K. (2000a). Mapping auditory hallucinations in schizophrenia using functional magnetic resonance imaging. Archives of General Psychiatry, 57, 1033-1038.

Shergill, S.S., Bullmore, E., Simmons, A., Murray, R., \& McGuare, P. (2000b). Functional anatomy of auditory verbal imagery in schizophrenic patients with auditory hallucinations. American Joumal of Psychiatry, 157, 1691-1693.

Shergill, S.S., Bullmore, E.T., Brammer, M.J., Williams, S.C.R., Murray, R.M., \& McGuire, P.K. (2001). A functional study of auditory verbal imagery. Psychological Medicine, $31,241-253$.

Shulman, G.L., Fiez, J.A., Corbetta, M., Buckner, R.L., Miezin, F.M., Raichle, M.E., \& Petersen, S.E. (1997). Common blood flow changes across visual tasks: II. Decreases in cerebral cortex. Journal of Cognitive Neuroscience, 9, 648-663.

Silbersweig, D.A., Stern, E., Frith, C., Cahill, C., Holmes, A., Grootoonk, S., et al., (1995). A functional neuroanatomy of hallucinations in schizophrenia. Nature, 378, 176179.

Silver, H., Feldman, P., Bilker, W., \& Gur, R.C. (2003). Working memory deficit as a core neuropsychological dysfunction in schizophrenia. American Joumal of Psychiatry, 160, 1809-1816.

Smith, D.B., Donchin, E., Cohen, L., \& Starx, A. (1970). Auditory averaged evoked potentials in man during selective binaural listening. Electroencephalography and Clinical Neurophysiology, 28, 146-152.

Spearman, C. (1923). The nature of "intelligence" and the principles of cognition. London: MacMillan.

Squires, N.K., Squires, K.C., \& Hillyard, S.A. (1975). Two varieties of long-latency positive waves evoked by unpredictable auditory stimuli in man. Electroencephalography and Clinical Neurophysiology, 38, 387-401.

Stark, C.E.L., \& Squire, L.R. (2001). When zero is not zero: The problem of ambiguous baseline conditions in FMRI. Proceedings of National Academy of Science USA, 98 . 12760-12766.

Starker, S., \& Jolin, A. (1982). Imagery and hallucinations in schizophrenic patients. Joumal of Neroous and Mental Disecase, 170, 448-451.

Stephane, M., Barton, S., \& Boutros, N.N. (2001). Auditory verbal hallucinations and dysfunction of the neural substrates of speechs. Schizophreria Research, 50, 61-78.

Stephane, M., Thuras, P.. Nasrallah, H., \& Georgopoulos, A.P. (2003). The internal structure of the phenomenology of auditory verbal hallucinations. Schizoplarenia Research, 61, 185-193.

Talairach, $J_{n}$ \& Tournoux, P. (1988). Co-planar stereotaxic atlas of the Hman brain. New York: Thieme Medical.

Thomas, C.G., Harshman, R.A., \& Menon, R.S. (2002). Noise reduction in BOLD-based fMRI using component analysis. Neuroimage, 17, 1521-1537.

Thurstone, L. L. (1938). The primary mental abilities. Chicago: University of Chicago Press. 


\section{References}

Torgenson, W. (1952). Multidmension scaling 1 - Theory and methods. Psychontetrics, 17, 401419 .

Trojano, $T_{\text {, }}$ Grossi, D., Linden, D.E.J., Formisano, E., Hacker, H., Zanella, F.E., Goebel, R. \& Di Salle, F. (2000). Matching two imagined clocks: the functional anatomy of patial analysis in the absence of visual stimulation. Cerebral Cortex, 10,473-481.

Tucker, D.M., Roth, D.L., \& Bair, T.B. (1986). Functional connections among corticall regions: Topography of EEG coherence. Electroencephatography and Clinical Neurophysiology, $63,242-250$.

Van de Ven, V. Merckelbach, H. (2003). The role of schizotypy, mental imagery and fantasy proneness in hallucinatory reports in undergraduate students. Personality and Indioidund Diferences, $35,889-896$.

Van de Ven, V.G., Formisano, E., Prvulovic, D., Roeder, C.H. \& Linden, D.E.J. (2004). Functional connectivity as revealed by spatial independent component analysis of AMRI measurements during rest. Human Brain Mapping, 22, 165-178.

Van de Vern, V.G., Sack, A.T., Linden, D.E.J., \& Formisano, E. (2002). Independent component analysis of $\mathrm{fMR}$ data: Identifying components of interest. Society for Neuroscience Abstract, 506.16.

Weickert, T.W., Goldberg, T.E., Gold, J.M., Bigelow, L.B., Egan, M.F., \& Weinberger, D.R. (2000\%. Cognitive impairments in patients with schizophrenia displaying preserved and compromised intellect. Archiwes of General Psychiatry, 57, 907-913.

Wheeler, M.E., Petersen, S.E., \& Buckner, R.L. (2000). Memory's echo: Vivid remembering reactivates sensory-specific cortex. Procedings of the National Academy of Science USA, 20, 11125-11129.

Wible, C.G., Kubicki, M., Yoo, S-S., Kacher, D.F, Salisbury, D.F, Anderson, M.C.,

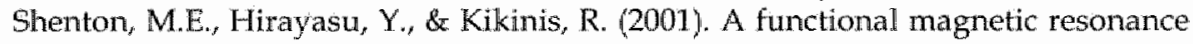
imaging study of auditory mismatch in schizophrenia. American Journal of Psyctivatry, 158, 938-943.

Woodruff, P.W.R., Wright, I.C., Bullmore, E.T., Brammer, M., Howard, R.I. \& Williams, S.C., (1997). Auditory hallucinations and the temporal cortical response to speech in schizophrenia: a functional magnetic resonance imaging study. American Joumal of Psychiatry, 154, 1676-1682.

World Health Organization (1992). Imtermational Statistical Classification of disenses and related heal h problems . Tenth Revision (ICD-10). WHO, Geneva

Xiong, I., Parsons, L.M., Gao, J.-H., \& Fox, P.T. (1999). Interregional connectivity to primary motor cortex revealed using MRI resting state images. Human Brain Mapping, 8, 151-156.

Yang, K. and Rajapakse, J.C. (2004). ICA gives higher-order functional connectivity of brain. Newral Information Processing - Letters and Rezicw, 2, 27-32. 


\section{Acknowledgements}

This thesis, while representing my progress and views on scientific matters, is also testimony to deeply appreciated support of many individuals. Although I cannot mention all, I do want to express my gratitude to the following people.

First and foremost, I am much indebted to David E.J. Linden, copromotor and supervisor , for taking me under his wings for more than two years in the Department of Psychiatry of the J.W. Goethe Universitäts Klinikum in Frankfurt am Main, Germany. He provided me with a unique and very stimulating working environment, and his unrelenting enthusiasm was an invaluable source of inspiration. For his guidance and support II am very gratefull.

I owe many thanks and gratitude to Elia Formisano for his support and his patience with me when I struggled to find my way through complicated data with a complicated method. Also, many thanks to Rainer Goebel for his support, enthusiasm, and not the least for bringing hallucinations and neuroimaging to my attention.

David Prvulovic, Roi Cohen Kadosh, Titia Gebuis, Anna Rotarska Jagiela and other (visiting) colleagues at the Department of Psychiatry and the Max Planck Institute for Brain Research made my stay in Frankfurt a great and informative experience, I thank them all for their enthusiasm, collaboration and friendship. Special thanks to Alex Sack for his help in locating a place to stay in the "best area of Frankfurt", and his company. Ik dank Christian Röder voor zijn gezelschap, kritische geest en interessante humor. En voor de wijn bij de discussies.

Christoph Bledowski machte unsere Aufenthalte in New York und Neapel zu lustigen und unvergesslichen Ereignissen. Ich bin sehr froh über unsere laufende Zusammenarbeit und hoffe auf neue spannende Kooperationen in der Zukunft. Und ich finde deine Literaturanregungen sehr bereichernd fur mich.

Much gratitude I also owe to my colleagues at the Universiteit Maastricht (including Milene Bonte, Amanda Kaas, Alex Sack, Anton de Vries, Judith Peters, Betina Sorger, Rainer Goebel, Alard Roebroeck, the Delphi-men Mart Bles and Michael Capalbo, Ingrid Christoffel, Federico Di Martino, Elia Formisano and Peter de Weerd). They form an exceptional group of people to work with, and have created an open, highly stimulating and very fun working and "craft" environment. Specifically, I would like to thank my "roomies", Joel Reithler and Lourens Waldrop, for patiently engaging into all kinds of endless 
discussions with me, and for tolerating my noisy taste of music. Bernadette Jansma made it possible for me to start my work in Frankfurt on hallucinations and ICA, and motherly came to my aid on several crucial occassions. I am much indebted for your support and advice. Ook ben ik veel dank verschuldigd aan Annemie Jeukens, voor haar geduld met mijn soms gebrekkige organisatie.

I thank Fabrizio Esposito, Fransceco Di Salle, and all the coworkers of the functional imaging laboratory in Naples, who helped me with understanding ICA, aided me in programming, and showed me around in the wonderous city of Naples. And of course for 'salsicce".

I gratefully acknowledge the cooperation of patients, caretakers and psychiatrists.

Scientific progress is achieved by critical thinking, deep thought, and muscle on the work flloor, but also by apparantly less direct influences from completely unrelated disciplines, most notably the shared experience and expression of music. Of great importance and fun were the violin classes that I enjoyed together with Henk, Hester, Peter and Marieke, as well as the musical meetings with colleagues and friends. Lastly, I want to thank the muzes David Gilmour, Saul Hudson, Zakk Wylde, Angus Young et all. for added noise and inspiring company.

Mijn dank gaat uit naar Fabian, JW en Dory, Robert en Josien, voor jullie wriendschap en steun, zelfs als ik maar niet kan leren mijn mobiel te gebruiken.

Ik dank mijn familie, Henk, Linda en Robin, voor hun steun. Meer dan dat bewonder ik hen voor hun ondernemingswil en veerkracht voor hetgeen hen drijft.

Finally, I am in great, if not eternal, debt to Mijntje Pieters, whose patience, sharp spirit and everlasting support are invaluable to me, and take me higher. 


\title{
Publications
}

\author{
Peer-reviewed journals
}

Merckelbach, H. \& Van de Ven, V. (2001). Another White Christmas: fantasy proneness and reports of 'hallucinatory experiences' in undergraduate students. Journal of Behavior Therapy and Experimental Psychiatry, 32, 137-144.

Van de Ven, V. \& Merckelbach, H. (2003). The role of schizotypy, mental imagery, and fantasy proneness in hallucinatory reports of undergraduate students. Personality and Indioidual Differences, 35, 889-896.

Van de Ven, V.G., Formisano, E., Prvulovic, D., Roeder, C. \& Linden, D.E.J. (2004). Functional connectivity as revealed by spatial independent component analysis of fMRI measurements during rest. Human Brain Mapping, 22, 165-178.

Sack, A.T., Van de Ven, V.G., Etschenberg, S., Schatz, D. \& Linden, D.E.J. (2005). Enhanced vividness as a trait marker for schizophrenia? Schizophrenia Bulletin, 1, 97-108.

Cohen Kadosh, R., Henik, A., Rubinsten, O., Mohr, H., Dori, H., Van de Ven, V., Zorzi, M., Hendler, $T_{. r}$ Goebel, R. \& Linden, D.E.J. (2005). Are numbers special? The comparison systems of the human brain investigated by fMRI. Neuropsychologia, 43 , 1238-1248.

Van de Ven, V.G., Formisano, E., Röder, C.H., Prvulovic, D., Bittner, R.A., Dietz, M.G., Daniela, H., Dierks, T., Federspiel, A., Esposito, F., Di Salle, F., Jansma, B., Goebel, R. \& Linden, D.E.J. (2005). The spatiotemporal pattern of auditory cortical responses during verbal hallucinations. Neuroinnge, 27, 644-655.

Prvulovic, D., Van de Ven, V., Sack, A.T., Maurer, K. \& Linden, D.E.J. (2005). Functional activation innaging in aging and dementia. Psychiatry Research: Neuroimaging, 140, $97-113$.

\section{Abstracts \& presentations}

V.G. van de Ven, A.T. Sack, D.E.J. Linden \& E. Formisano. (2002). Identification of components of interest. Society for Neuroscience, Orlando, Florida.

V.G. van de Ven, E. Formisano, D. Prvulovic, C. Roeder, R. Bittner, M.G. Dietz, D. Hubl, T. Dierks, A. Federspiel, R. Goebel \& D.E.J. Linden. (2003). Cortical responses of auditory hallucinations analysed using hypothesis- and data-driven methods: A first approach [abstract]. Presented at the $9^{\text {th }}$ International Conference on Functional Mapping of the Human Brain, June 19-22, 2003, New York, NY. Available on CD-ROM in Neuroimage, $19(2)$. 
D.E. Linden, V. van de Ven, S. Etschenberg, D. Schatz \& A.T. Sack (2003). Enhanced vividness of mental imagery as a trait marker of schizophrenia? International Congress on Schizophrenia Research 2003, Colorado Springs, CO, USA. Schizophrenia Research, 60, suppl. 1, 20 .

R. Cohen Kadosh, A. Henik, O. Rubinsten, H. Dori, Y. Bloch-David, H. Mohr, V. van de Ver, M. Zorzi, T. Hendler \& D.E. J. Linden. (2003). Are Numbers Special? A Common System for Numerical and Physical Comparisons in the Parietal Lobes. Cognitive Neuroscience Conference, New York.

Van de Ven, V.G., Bledowski, C., Pvrulovic, D., Goebel, R., Formisano, E., Linden, D.E., Di Salle, F. \& Esposito, F. (2005). Components of target detection revealed by fMRI using self-organizing group-levell ICA [abstract]. Presented at the $11^{\text {th }}$ International Conference on Functional Mapping of the Human Brain, June 11-17, 2005, Toronto.

Van de Ven, V., Formisano, E., Goebel, R., Prvulovic, D., Roder, C. \& Linden, D.E.J. (2005). Analyzing neural networks of auditory verbal hallucinations. Nederlandse Vereniging voor Psychonomie Wintercongres 2005. 